\title{
The unitary conformal field theory behind 2D Asymptotic Safety
}

\author{
Andreas Nink and Martin Reuter \\ Institute of Physics, PRISMA \& MITP, Johannes Gutenberg University Mainz, \\ Staudingerweg 7, D-55099 Mainz, Germany \\ E-mail: nink@thep.physik.uni-mainz.de, \\ reuter@thep.physik.uni-mainz.de
}

ABstract: Being interested in the compatibility of Asymptotic Safety with Hilbert space positivity (unitarity), we consider a local truncation of the functional RG flow which describes quantum gravity in $d>2$ dimensions and construct its limit of exactly two dimensions. We find that in this limit the flow displays a nontrivial fixed point whose effective average action is a non-local functional of the metric. Its pure gravity sector is shown to correspond to a unitary conformal field theory with positive central charge $c=25$. Representing the fixed point CFT by a Liouville theory in the conformal gauge, we investigate its general properties and their implications for the Asymptotic Safety program. In particular, we discuss its field parametrization dependence and argue that there might exist more than one universality class of metric gravity theories in two dimensions. Furthermore, studying the gravitational dressing in 2D asymptotically safe gravity coupled to conformal matter we uncover a mechanism which leads to a complete quenching of the a priori expected Knizhnik-Polyakov-Zamolodchikov (KPZ) scaling. A possible connection of this prediction to Monte Carlo results obtained in the discrete approach to 2D quantum gravity based upon causal dynamical triangulations is mentioned. Similarities of the fixed point theory to, and differences from, non-critical string theory are also described. On the technical side, we provide a detailed analysis of an intriguing connection between the Einstein-Hilbert action in $d>2$ dimensions and Polyakov's induced gravity action in two dimensions.

KeYwords: Models of Quantum Gravity, 2D Gravity, Nonperturbative Effects, Renormalization Group

ARXIV EPRINT: 1512.06805 


\section{Contents}

1 Introduction 1

2 Background independence via background fields 5

2.1 The effective Einstein equation re-interpreted 5

$\begin{array}{ll}2.2 & \text { The stress tensor of the } h_{\mu \nu} \text {-fluctuations }\end{array}$

2.3 The intrinsic description in exactly 2 dimensions 8

3 How the induced gravity action emerges from the Einstein-Hilbert action 9

$\begin{array}{ll}3.1 \text { Lessons from the conformal gauge } & 11\end{array}$

$\begin{array}{ll}3.2 \text { General properties of the limit } & 13\end{array}$

$\begin{array}{lll}3.3 & \text { Establishing the 2D limit } & 15\end{array}$

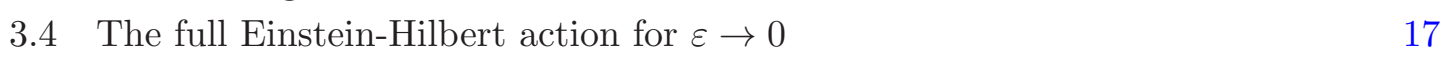

$\begin{array}{ll}3.5 & \text { Aside: is there a generalization to } 4 \mathrm{D} ?\end{array}$

4 The NGFP as a conformal field theory $\quad 20$

5 Status of different field parametrizations $\quad \mathbf{2 9}$

$\begin{array}{lll}5.1 & \text { Different universality classes? } & 29\end{array}$

5.2 The birth of exponentials in 2D 30

6 The reconstructed functional integral $\quad 34$

6.1 The reconstruction process 34

6.2 A functional integral for 2D asymptotically safe gravity 35

$\begin{array}{ll}6.3 \text { Comparison with Monte Carlo results } & 40\end{array}$

$\begin{array}{lll}7 & \text { Conclusions } & 41\end{array}$

A Weyl transformations, zero modes and the induced gravity action $\quad 43$

A.1 The induced gravity action in the absence of zero modes 45

A.2 The treatment of zero modes 46

\section{Introduction}

During the past two decades, Asymptotic Safety [1] matured from a hypothetical scenario to a theory with a realistic chance to describe the structure of spacetime and the gravitational interaction consistently and predictively even on the shortest length scales possible. In particular, there is mounting evidence supporting the existence of the decisive nontrivial renormalization group (RG) fixed point. Yet, a number of immediate questions are still 
open. The most obvious one is about the precise nature of the action functional which describes this fixed point. In which way exactly does it depend on the metric, the background metric, and the Faddeev-Popov ghosts? Is it local? What are the structural properties of the fixed point theory, i.e. the one defined directly at the fixed point rather than by a trajectory running away from it. Is this theory a conformal field theory?

In 2 dimensions we are indeed used to the picture that the conformal field theories correspond to points in theory space that are fixed points of the RG flow [2]. In 4 dimensions, however, Quantum Einstein Gravity (QEG) has a scale invariant fixed point theory but it is unclear whether it is conformal.

While conformality is not known to be indispensable, there exist several other properties an asymptotically safe theory must possess in addition to its mere nonperturbative renormalizability, that is, the existence of a suitable non-Gaussian fixed point. The two most important ones are clearly Background Independence and unitarity. While there are by now first promising results which indicate that the requirements of Background Independence and Asymptotic Safety can be met simultaneously in sufficiently general truncations of the RG flow [3], little is known about the status of unitarity.

In this connection the somewhat colloquial term "unitarity" is equivalent to "Hilbert space positivity" and is meant to express that the state space of the system under consideration contains no vectors having a negative scalar product with itself ("negative norm states"). If it does so, it is not a Hilbert space in the mathematical sense of the word and cannot describe a quantum system as the probability interpretation of quantum mechanics would break down then.

At least on (nondynamical) flat spacetimes the criterion of Hilbert space positivity, alongside with the spectral condition can be translated from the Lorentzian to the Euclidean setting where it reappears as the requirement of reflection-, or Osterwalder-Schrader, positivity $[4-7]$.

Unitarity is indeed a property that is not automatic and needs to be checked in order to demonstrate the viability of the Asymptotic Safety program based upon the Effective Average Action (EAA). The EAA for gravity [8] is a scale dependent effective action for a BRST gauge-fixed theory. Its operator formulation amounts to an indefinite metric (Krein space) quantization, and so the negative norm states it contains should be "factored out" ultimately in order to obtain a positive ("physical") state space, a true Hilbert space. While this procedure is standard and familiar from perturbative quantum gravity and Yang-Mills theory, for instance, the situation is much more involved in Asymptotic Safety. The reason is that, implicitly, this indefinite metric quantization is applied to a bare action which is essentially given by the fixed point functional [9-12]. As such it is already in itself the result of a technically hard nonperturbative computation which in practice can be done only approximately, for the time being.

In the present paper we explore the question of Hilbert space positivity together with a number of related issues such as locality by analyzing the situation in 2 dimensions where a number of technical simplifications occur. We start out from the Einstein-Hilbert truncation of the EAA in $d=2+\varepsilon>2$ dimensions, investigate the nature of its $\varepsilon \rightarrow 0$ limit, and finally construct a manifestly 2-dimensional action which describes 2D Asymptotic 
Safety without reverting to "higher" dimensions in any way. In this manner we shall see that the non-Gaussian fixed point (NGFP) underlying Asymptotic Safety is governed by a conformal field theory (CFT) which is interesting in its own right, and whose properties we shall discuss. Interestingly enough, it turns out to possess a positive central charge, thus giving rise to a unitary representation of the Virasoro algebra and a "positive" Hilbert space in the above sense.

(1). A framework which allows to systematically search for asymptotically safe theories is the Effective Average Action, and in particular its functional RG equation (FRGE): the RG flow of the EAA, $\Gamma_{k}$, is governed by the exact nonperturbative evolution equation $[8,13-17]$

$$
k \partial_{k} \Gamma_{k}=\frac{1}{2} \operatorname{STr}\left[\left(\Gamma_{k}^{(2)}+\mathcal{R}_{k}\right)^{-1} k \partial_{k} \mathcal{R}_{k}\right] .
$$

It describes the dependence of $\Gamma_{k}$ on the RG scale $k$ which plays the role of a regularization scale for infrared (IR) fluctuations. The suppression of IR modes is realized by the cutoff operator $\mathcal{R}_{k}$, satisfying $\mathcal{R}_{k} \rightarrow k^{2}$ for IR and $\mathcal{R}_{k} \rightarrow 0$ for UV modes, respectively. Besides, $\Gamma_{k}^{(2)}$ denotes the Hessian of the EAA with respect to the dynamical field. In particular, in the case of gravity when the background field method is employed, it is the second functional derivative of $\Gamma_{k}[g, \bar{g}]$ w.r.t. $g$ at fixed background $\bar{g}$. By construction, $\Gamma_{k}$ approaches the full quantum effective action $\Gamma$ in the limit $k \rightarrow 0$. Although its $k \rightarrow \infty$ limit is formally related to the bare action [15-17], in the Asymptotic Safety program no bare action is posited a priori; it is rather "reconstructed" from the condition that this limit actually exists nonperturbatively [9-12].

In order to find solutions to the FRGE (1.1) one usually resorts to truncations, implying a reduction of the infinite dimensional theory space that consists of all possible action monomials compatible with the underlying symmetry. By now a large variety of truncations have been studied to support the existence of a NGFP of metric gravity, including for instance terms of higher orders in the curvature or actions that couple gravity to matter [18-21].

(2). In this paper we will focus on a gravity + matter system where the purely gravitational sector consists of the Euclidean Einstein-Hilbert truncation,

$$
\Gamma_{k}^{\text {grav }}[g]=\frac{1}{16 \pi G_{k}} \int \mathrm{d}^{d} x \sqrt{g}\left(-R+2 \Lambda_{k}\right),
$$

and the matter contribution is given by a multiplet of $N$ scalar fields $A=\left(A^{i}\right)$, with $i=1, \cdots, N$, minimally coupled to the full, dynamical metric:

$$
\Gamma_{k}^{\mathrm{M}}[g, A] \equiv \Gamma^{\mathrm{M}}[g, A]=\frac{1}{2} \sum_{i=1}^{N} \int \mathrm{d}^{d} x \sqrt{g} g^{\mu \nu} \partial_{\mu} A^{i} \partial_{\nu} A^{i} .
$$

Note that the matter action contains no running parameters in the present truncation(s). Supplemented by appropriate gauge fixing and ghost terms, the sum of $\Gamma_{k}^{\text {grav }}$ and $\Gamma_{k}^{\mathrm{M}}$ can be inserted into the flow equation (1.1) in order to determine the running of Newton's constant $G_{k}$ and the cosmological constant $\Lambda_{k}$. 
The RG studies of this system for $d=2+\varepsilon$ dimensions $(\varepsilon \searrow 0)$ are of particular importance for our analysis. It is possible then to argue on general grounds that the $\beta$-function of the dimensionless Newton constant $g_{k} \equiv G_{k} k^{d-2}=G_{k} k^{\varepsilon}$ must be of the form

$$
\beta_{g}=\varepsilon g-b g^{2}+\mathcal{O}\left(g^{3}\right)
$$

This implies the existence of a nontrivial fixed point which is located at

$$
g_{*}=\varepsilon / b,
$$

up to higher $\varepsilon$-orders. Therefore, we have $g_{k} \propto \varepsilon$ and, in turn, $G_{k} \propto \varepsilon$ in the vicinity of the NGFP, having far-reaching consequences for the dimensional continuation of the Einstein-Hilbert action to two dimensions.

(3). In exactly 2 dimensions the integral $\int \mathrm{d}^{2} x \sqrt{g} R$ is a purely topological term according to the Gauss-Bonnet theorem. In particular, it is independent of the metric and does not imply any local dynamics. Thus, one might expect that (1.2) becomes trivial up to the cosmological constant term when $d$ approaches 2 . However, the fact that the prefactor $1 / G_{k}$ entails a $1 / \varepsilon$ pole gives so much weight to $\int \mathrm{d}^{2+\varepsilon} x \sqrt{g} R$ that the limit $\varepsilon \rightarrow 0$ remains indeed nontrivial.

We will present a new argument in this work showing that the (local) Einstein-Hilbert action turns into a non-local action in the limit $d \rightarrow 2$. The essential part of this limit action will be seen to be given by Polyakov's induced gravity action.

Our proof will confirm recurring speculation [22] that the induced gravity action is the natural 2-dimensional analogue of the Einstein-Hilbert action in $d>2$ as both actions determine field equations for the metric in their respective spacetime dimension.

(4). Here we go one step further: we do not require that one action has to be replaced by the other one when switching between $d=2$ and $d>2$. The idea is rather to say that there is only one common origin, the Einstein-Hilbert action in a general dimension $d$, and that the induced gravity action emerges automatically when d approaches 2 .

It is this latter 2D action, analyzed at the NGFP, that establishes the contact between the Asymptotic Safety studies within the Einstein-Hilbert truncation and 2-dimensional conformal field theory. It will form the basis of our investigations concerning central charges and unitarity.

(5). It turns out that the crucial fixed point value $g_{*}$ depends on the way the metric is parametrized. The background field technique which underlies the Asymptotic Safety studies expresses the dynamical metric $g_{\mu \nu}$ in terms of a fixed background metric $\bar{g}_{\mu \nu}$ and the fluctuations $h_{\mu \nu}$. There are, however, different possible choices of how these fields are related, in particular the standard linear parametrization $g_{\mu \nu}=\bar{g}_{\mu \nu}+h_{\mu \nu}$ and the exponential parametrization $g_{\mu \nu}=\bar{g}_{\mu \rho}\left(\mathrm{e}^{h}\right)^{\rho}$. These two parametrizations give rise to different $\beta$-functions and different central charges. As we will argue, this disagreement does not necessarily mean that one choice is correct while the other one is wrong, and we advocate the possibility that the two parametrizations might refer to different universality classes. 
In the $2 \mathrm{D}$ limit, however, we provide strong evidence that the exponential parametrization is more appropriate for a consistent description of the gravitational interactions. It is this choice that leads to a NGFP theory with the value $c_{\text {grav }}=25$ for the central charge of the pure gravity sector.

(6). This paper is organized as follows. We review Background Independence and the special role of self-consistent backgrounds in section 2. In particular, we re-interpret the effective Einstein equation as a tadpole condition and the trace of the stress energy tensor due to metric fluctuations as a kind of classical "trace anomaly". Here, all calculations are performed in $2+\varepsilon$ dimensions, and the $2 \mathrm{D}$ limit is taken at the very end only. This leads us to the question if the same trace anomaly can be obtained when starting out from a strictly $2 \mathrm{D}$ action. The answer to this question will be given in section 3 where we compute the 2D limit of the Einstein-Hilbert action at the NGFP and argue that it results indeed in an action with the sought-for properties. We demonstrate in section 4 that this $2 \mathrm{D}$ gravitational NGFP action gives rise to a unitary conformal field theory. Particular attention is paid to the relation between the crucial sign of its central charge, the occurrence of a conformal factor instability, and unitarity. Section 5 is dedicated to the impact of different metric parametrizations and establishes the special status of the exponential parametrization in the 2D limit. Finally, section 6 is devoted to the properties of the fixed point CFT, and a comparison of Asymptotic Safety to other approaches to 2D gravity. For that purpose we construct a functional integral which reproduces the effective average action related to the CFT behind the NGFP. This "reconstructed" functional integral is used to investigate the gravitational dressing of matter field operators when conformal matter is coupled to asymptotically safe gravity. We demonstrate that, contrary to what one would have expected, there is no KPZ scaling in our setting as a consequence of Asymptotic Safety. We also discuss similarities and differences compared with non-critical string theory and Monte-Carlo simulations in the CDT approach. Section 7 contains our conclusions and an outlook.

In the appendix we catalog various useful identities for Weyl transformations, and we include a detailed discussion of the induced gravity action in the presence of zero modes.

\section{Background independence via background fields}

In this preparatory section we collect a number of results concerning the implementation of Background Independence in the EAA framework which actually does employ (unspecified!) background fields. In particular, we introduce the energy momentum tensor of metric fluctuations in a background, as well as an associated "trace anomaly". The latter will be used later on in order to identify the conformal field theory at the heart of Asymptotic Safety in 2 dimensions.

\subsection{The effective Einstein equation re-interpreted}

Let us consider a generic effective average action $\Gamma_{k}[\Phi, \bar{\Phi}] \equiv \Gamma_{k}[\varphi ; \bar{\Phi}]$ involving a multiplet of dynamical fields $\left\langle\hat{\Phi}^{i}\right\rangle \equiv \Phi^{i}$, associated background fields $\bar{\Phi}^{i}$, and fluctuations $\varphi^{i} \equiv$ $\left\langle\hat{\varphi}^{i}\right\rangle=\Phi^{i}-\bar{\Phi}^{i}$. The effective average action implies a source $\leftrightarrow$ field relationship which 
contains an explicit cutoff term linear in the fluctuation fields:

$$
\frac{1}{\sqrt{\bar{g}}} \frac{\delta \Gamma_{k}[\varphi ; \bar{\Phi}]}{\delta \varphi^{i}(x)}+\mathcal{R}_{k}[\bar{\Phi}]_{i j} \varphi^{j}(x)=J_{i}(x) .
$$

By definition, self-consistent backgrounds are field configurations $\bar{\Phi}(x) \equiv \bar{\Phi}_{k}^{\mathrm{sc}}(x)$ which allow $\varphi^{i}=0$ to be a solution of (2.1) with $J_{i}=0$. A self-consistent background is particularly "liked" by the fluctuations, in the sense that they leave it unaltered on average: $\langle\hat{\Phi}\rangle=\bar{\Phi}+\langle\hat{\varphi}\rangle=\bar{\Phi}^{\text {sc }}$. These special backgrounds are determined by the tadpole condition $\left\langle\hat{\varphi}^{i}\right\rangle=0$, which reads explicitly

$$
\left.\frac{\delta}{\delta \varphi^{i}(x)} \Gamma_{k}[\varphi ; \bar{\Phi}]\right|_{\varphi=0, \bar{\Phi}=\bar{\Phi}_{k}^{\text {sc }}}=0
$$

Equivalently, in terms of the full dynamical field,

$$
\left.\frac{\delta}{\delta \Phi^{i}(x)} \Gamma_{k}[\Phi, \bar{\Phi}]\right|_{\Phi=\bar{\Phi}=\bar{\Phi}_{k}^{\text {sc }}}=0 .
$$

In this paper we consider actions of the special type

$$
\Gamma_{k}[g, \xi, \bar{\xi}, A, \bar{g}]=\Gamma_{k}^{\mathrm{grav}}[g, \bar{g}]+\Gamma_{k}^{\mathrm{M}}[g, A, \bar{g}]+\Gamma_{k}^{\mathrm{gf}}[g, \bar{g}]+\Gamma_{k}^{\mathrm{gh}}[g, \xi, \bar{\xi}, \bar{g}] .
$$

These functionals include a purely gravitational piece, $\Gamma_{k}^{\text {grav }}$, furthermore a (for the time being) generic matter action $\Gamma_{k}^{\mathrm{M}}$, as well as gauge fixing and ghost terms, $\Gamma_{k}^{g f}$ and $\Gamma_{k}^{\text {gh }}$, respectively. Concerning the latter, only the following two properties are needed at this point: (i) The $h_{\mu \nu}$-derivative of the gauge fixing functional $\Gamma_{k}^{\mathrm{gf}}[h ; \bar{g}] \equiv \Gamma_{k}^{\mathrm{gf}}[\bar{g}+h, \bar{g}]$ vanishes at $h_{\mu \nu}=0$. This is the case, for example, for classical gauge fixing terms $S_{\text {gf }} \propto \int(\mathcal{F} h)^{2}$ which are quadratic in $h_{\mu \nu}$. (ii) The functional $\Gamma_{k}^{\text {gh }}$ is ghost number conserving, i.e. all terms contributing to it have an equal number of ghosts $\xi^{\mu}$ and antighosts $\bar{\xi}_{\mu}$. Again, classical ghost kinetic terms $\propto \int \bar{\xi} \mathcal{M} \xi$ are of this sort.

Thanks to these properties, $\Gamma_{k}^{\mathrm{gf}}$ drops out of the tadpole equation (2.3), and it follows that $\xi=0=\bar{\xi}$ is always a consistent background for the Faddeev-Popov ghosts. Adopting this background for the ghosts, (2.3) boils down to the following conditions for self-consistent metric and matter field configurations $\bar{g}_{k}^{\mathrm{sc}}$ and $\bar{A}_{k}^{\mathrm{sc}}$, respectively:

$$
\begin{aligned}
& 0=\left.\frac{\delta}{\delta g_{\mu \nu}(x)}\left\{\Gamma_{k}^{\mathrm{grav}}[g, \bar{g}]+\Gamma_{k}^{\mathrm{M}}\left[g, \bar{A}_{k}^{\mathrm{sc}}, \bar{g}\right]\right\}\right|_{g=\bar{g}=\bar{g}_{k}^{\mathrm{sc}}}, \\
& 0=\left.\frac{\delta}{\delta A(x)} \Gamma_{k}^{\mathrm{M}}[g, A, \bar{g}]\right|_{g=\bar{g}=\bar{g}_{k}^{\mathrm{sc}}, A=\bar{A}_{k}^{\mathrm{sc}}} .
\end{aligned}
$$

Introducing the energy-momentum tensor of the matter field,

$$
\left.T^{\mathrm{M}}[\bar{g}, A]^{\mu \nu}(x) \equiv \frac{2}{\sqrt{\bar{g}(x)}} \frac{\delta}{\delta g_{\mu \nu}(x)} \Gamma_{k}^{\mathrm{M}}[g, A, \bar{g}]\right|_{g=\bar{g}},
$$

the first condition, equation (2.5), becomes

$$
0=\left.\frac{2}{\sqrt{\bar{g}(x)}} \frac{\delta}{\delta g_{\mu \nu}(x)} \Gamma_{k}^{\mathrm{grav}}[g, \bar{g}]\right|_{g=\bar{g}=\bar{g}_{k}^{\mathrm{sc}}}+T^{\mathrm{M}}\left[\bar{g}_{k}^{\mathrm{sc}}, \bar{A}_{k}^{\mathrm{sc}}\right]^{\mu \nu}(x) .
$$


This relation plays the role of an effective gravitational field equation which, together with the matter equation (2.6), determines $\bar{g}_{k}^{\text {sc }}$ and $\bar{A}_{k}^{\text {sc }}$. Structurally, eq. (2.8) is a generalization of the classical Einstein equation to which it reduces if $\Gamma_{k}^{\text {grav }}[g, \bar{g}] \equiv \Gamma_{k}^{\text {grav }}[g]$ happens to have no "extra $\bar{g}$-dependence" [23] and to coincide with the Einstein-Hilbert action; then the $\delta / \delta g_{\mu \nu}$-term in (2.8) is essentially the Einstein tensor $G_{\mu \nu}$.

In this very special background-free case we recover the familiar setting of classical General Relativity where there is a clear logical distinction between matter fields and the metric, meaning the full one, $g_{\mu \nu}$, while none other appears in the fundamental equations then. It is customary to express this distinction by putting $G_{\mu \nu}$ on the l.h.s. of Einstein's equation, the side of gravity, and $T_{\mu \nu}^{\mathrm{M}}$ on the r.h.s., the side of matter.

In the effective average action approach where, for both deep conceptual and technical reasons [3, 23], the introduction of a background is unavoidable during the intermediate calculational steps, this categorical distinction of matter and gravity, more precisely, matter fields and metric fluctuations, appears unmotivated. It is much more natural to think of $h_{\mu \nu}$ as a matter field which propagates on a background spacetime furnished with the metric $\bar{g}_{\mu \nu}$.

Adopting this point of view, we interpret the $\delta / \delta g_{\mu \nu}$-term in (2.8) as the energymomentum tensor of the $h_{\mu \nu}$-field, and we define

$$
\left.T^{\text {grav }}[\bar{g}]^{\mu \nu}(x) \equiv \frac{2}{\sqrt{\bar{g}(x)}} \frac{\delta}{\delta g_{\mu \nu}(x)} \Gamma_{k}^{\text {grav }}[g, \bar{g}]\right|_{g=\bar{g}}=\left.\frac{2}{\sqrt{\bar{g}(x)}} \frac{\delta}{\delta h_{\mu \nu}(x)} \Gamma_{k}^{\text {grav }}[h ; \bar{g}]\right|_{h=0} .
$$

The tadpole equation (2.8) turns into an Einstein equation with zero l.h.s. then:

$$
0=T_{\mu \nu}^{\mathrm{grav}}\left[\bar{g}_{k}^{\mathrm{sc}}\right]+T_{\mu \nu}^{\mathrm{M}}\left[\bar{g}_{k}^{\mathrm{sc}}, \bar{A}_{k}^{\mathrm{sc}}\right] .
$$

It says that for a background to be self-consistent, the total energy-momentum tensor of matter and metric fluctuations, in this background, must vanish. (In the general case there could be a contribution from the ghosts also.)

\subsection{The stress tensor of the $h_{\mu \nu}$-fluctuations}

Note that in general, $T_{\mu \nu}^{\text {grav }}$ is not conserved, $\bar{D}_{\mu} T^{\text {grav }}[\bar{g}]^{\mu \nu} \neq 0$, since due to the presence of two fields in $\Gamma_{k}^{\text {grav }}$ the standard argument does noes not apply. Of course, it is conserved in the special case $\Gamma_{k}^{\text {grav }}[g, \bar{g}] \equiv \Gamma_{k}^{\text {grav }}[g]$ when there is no extra $\bar{g}$-dependence.

For example, choosing $\Gamma_{k}^{\text {grav }}[\bar{g}]$ to be the single-metric Einstein-Hilbert functional (1.2), the corresponding energy-momentum tensor of the $h_{\mu \nu}$-fluctuations is given by the divergence-free expression

$$
T_{\mu \nu}^{\text {grav }}[\bar{g}]=\frac{1}{8 \pi G_{k}}\left(\bar{G}_{\mu \nu}+\Lambda_{k} \bar{g}_{\mu \nu}\right)
$$

with $\bar{G}_{\mu \nu}$ the Einstein tensor built from $\bar{g}_{\mu \nu}$. The trace of the energy-momentum tensor $(2.11)$ reads

$$
\Theta_{k}[\bar{g}] \equiv \bar{g}^{\mu \nu} T_{\mu \nu}^{\mathrm{grav}}[\bar{g}]=\frac{1}{16 \pi G_{k}}\left[-(d-2) \bar{R}+2 d \Lambda_{k}\right]
$$


where $\bar{R} \equiv R(\bar{g})$. A remarkable feature of this trace is that it possesses a completely well defined, unambiguous limit $d \rightarrow 2$ if $G_{k}$ and $\Lambda_{k}$ are of first order in $\varepsilon=d-2$. In terms of the finite quantities $\breve{G}_{k} \equiv G_{k} / \varepsilon$ and $\breve{\Lambda}_{k} \equiv \Lambda_{k} / \varepsilon$ which are of order $\varepsilon^{0}$, we have

$$
\begin{aligned}
\Theta_{k}[\bar{g}] & =\frac{1}{16 \pi \breve{G}_{k}}\left[-\bar{R}+4 \breve{\Lambda}_{k}\right]+\mathcal{O}(\varepsilon) \\
& =\frac{1}{16 \pi \breve{g}_{k}}\left[-\bar{R}+4 k^{2} \breve{\lambda}_{k}\right]+\mathcal{O}(\varepsilon) .
\end{aligned}
$$

In the second line of (2.13) we exploited that in exactly two dimensions the dimensionful and dimensionless Newton constant are equal, so $g_{k}=G_{k}$ and $\breve{g}_{k}=\breve{G}_{k}$, while, as always, $\lambda_{k} \equiv \Lambda_{k} / k^{2}$, hence $\breve{\lambda}_{k}=\breve{\Lambda}_{k} / k^{2}$.

When the underlying RG trajectory is in the NGFP scaling regime the dimensionless couplings are scale independent, and

$$
\Theta_{k}^{\mathrm{NGFP}}[\bar{g}]=\frac{1}{16 \pi \breve{g}_{*}}\left[-\bar{R}+4 \breve{\lambda}_{*} k^{2}\right] .
$$

Using the parametrization $g_{*} \equiv \varepsilon / b$ as in references $[3,24-26]$ we obtain

$$
\Theta_{k}^{\mathrm{NGFP}}[\bar{g}]=\left(\frac{3}{2} b\right) \frac{1}{24 \pi}\left[-\bar{R}+4 \breve{\lambda}_{*} k^{2}\right] .
$$

Here and in the following we consider $\Theta_{k}$ and $\Theta_{k}^{\mathrm{NGFP}}$ as referring to exactly 2 dimensions, in the sense that the limit has already been taken, and we omit the "O $\mathcal{O}(\varepsilon)$ " symbol.

\subsection{The intrinsic description in exactly 2 dimensions}

In this paper we would like to describe the limit $d \rightarrow 2$ of QEG in an intrinsically 2dimensional fashion, that is, in terms of a new functional $\Gamma_{k}^{\text {grav, } 2 \mathrm{D}}$ whose arguments are fields in strictly 2 dimensions, and which no longer makes reference to its "higher" dimensional origin. Since the Einstein-Hilbert term is purely topological in exactly $d=2$, it is clear that the sought-for action must have a different structure.

(1). One of the conditions which we impose on $\Gamma_{k}^{\text {grav }, 2 D}$ is that it must reproduce the trace $\Theta_{k}$ computed in $d>2$, since we saw that this quantity has a smooth limit with an immediate interpretation in $d=2$ exactly:

$$
\left.2 g_{\mu \nu} \frac{\delta}{\delta g_{\mu \nu}} \Gamma_{k}^{\text {grav }, 2 \mathrm{D}}[g, \bar{g}]\right|_{g=\bar{g}}=\sqrt{\bar{g}} \Theta_{k}[\bar{g}] .
$$

Furthermore, if $\Gamma_{k}^{\text {grav }}$ is a single-metric action, we assume that $\Gamma_{k}^{\text {grav,2D }} \equiv \Gamma_{k}^{\text {grav,2D }}[g]$ has no extra $\bar{g}$-dependence either. The condition (2.16) fixes its response to an infinitesimal Weyl transformation then:

$$
\left.2 g_{\mu \nu}(x) \frac{\delta}{\delta g_{\mu \nu}(x)} \Gamma_{k}^{\text {grav }, 2 \mathrm{D}}[g] \equiv \frac{\delta}{\delta \sigma(x)} \Gamma_{k}^{\text {grav }, 2 \mathrm{D}}\left[\mathrm{e}^{2 \sigma} g\right]\right|_{\sigma=0}=\sqrt{g(x)} \Theta_{k}[g](x) .
$$

For the example of the Einstein-Hilbert truncation, $\Theta_{k}$ is of the form

$$
\Theta_{k}[g]=a_{1}\left(-R+a_{2}\right),
$$

with constants $a_{1}, a_{2}$ which can be read off from (2.13)-(2.15) for the various cases. 
(2). It is well known how to integrate equation (2.17) in the conformal gauge [27]. By setting

$$
g_{\mu \nu}(x)=\hat{g}_{\mu \nu}(x) \mathrm{e}^{2 \phi(x)},
$$

with a fixed reference metric $\hat{g}_{\mu \nu}$ (conceptually unrelated to $\bar{g}_{\mu \nu}$ ), one for each topological sector, and taking advantage of the identities in appendix A, eq. (2.17) with (2.18) is turned into

$$
\frac{\delta}{\delta \phi(x)} \Gamma_{k}^{\text {grav }, 2 \mathrm{D}}\left[\mathrm{e}^{2 \phi} \hat{g}\right]=a_{1} \sqrt{\hat{g}(x)}\left[2 \hat{D}_{\mu} \hat{D}^{\mu} \phi(x)-\hat{R}(x)+a_{2} \mathrm{e}^{2 \phi(x)}\right]
$$

The general solution to this equation is easy to find:

$$
\Gamma_{k}^{\text {grav }, 2 \mathrm{D}}\left[\hat{g} \mathrm{e}^{2 \phi}\right]=\Gamma_{k}^{\mathrm{L}}[\phi ; \hat{g}]+U_{k}[\hat{g}] .
$$

Here $U_{k}$ is a completely arbitrary functional of $\hat{g}$, independent of $\phi$, and $\Gamma_{k}^{\mathrm{L}}$ denotes the Liouville action [28]:

$$
\begin{aligned}
\Gamma_{k}^{\mathrm{L}}[\phi ; \hat{g}] & =\left(-2 a_{1}\right) \int \mathrm{d}^{2} x \sqrt{\hat{g}}\left(\frac{1}{2} \hat{D}_{\mu} \phi \hat{D}^{\mu} \phi+\frac{1}{2} \hat{R} \phi-\frac{a_{2}}{4} \mathrm{e}^{2 \phi}\right) \\
& =\left(-2 a_{1}\right) \Delta I[\phi ; \hat{g}]+\frac{1}{2} a_{1} a_{2} \int \mathrm{d}^{2} x \sqrt{\hat{g}} \mathrm{e}^{2 \phi} .
\end{aligned}
$$

In the last line we employed the normalized functional

$$
\Delta I[\phi ; g] \equiv \frac{1}{2} \int \mathrm{d}^{2} x \sqrt{g}\left(D_{\mu} \phi D^{\mu} \phi+R \phi\right) .
$$

While this method of integrating the trace "anomaly" applies in all topological sectors, it is unable to find the functional $U_{k}[\hat{g}]$. Usually in conformal field theory or string theory this is not much of a disadvantage, but in quantum gravity where Background Independence is a pivotal issue it is desirable to have a more complete understanding of $\Gamma_{k}^{\text {grav }, 2 \mathrm{D}}$. For this reason we discuss in the next section the possibility of taking the limit $\varepsilon \rightarrow 0$ directly at the level of the action.

\section{How the induced gravity action emerges from the Einstein-Hilbert action}

In this section we reveal a mechanism which allows us to regard Polyakov's induced gravity action in 2 dimensions as the $\varepsilon \rightarrow 0$ limit of the Einstein-Hilbert action in $2+\varepsilon$ dimensions. Here and in the following we always consider the case $\varepsilon>0$, i.e. the limit $\varepsilon \searrow 0$. This will confirm the point of view that the induced gravity action is fundamental in describing 2-dimensional gravity, while it is less essential for $d>2$ where gravity is governed mainly by an (effective average) action of Einstein-Hilbert type. The dimensional limit exhibits a discontinuity at $d=2$, producing a non-local action out of a local one. 
(1). The crucial ingredient for a nontrivial limit $\varepsilon \rightarrow 0$ is a prefactor of the EinsteinHilbert action proportional to $1 / \varepsilon$. This occurs whenever the Newton constant is proportional to $\varepsilon$. As mentioned in the introduction, such a behavior was found in the Asymptotic Safety related RG studies. The renormalization group flow has a non-Gaussian fixed point with a Newton constant of order $\varepsilon$; a result which is independent of the underlying regularization scheme and which is found in both perturbative and nonperturbative investigations.

Employing a reconstruction formula [9, 12, 29] we shall see that this property holds not only for the effective, but also for the bare action: using an appropriate regularization prescription the bare Newton constant is of first order in $\varepsilon$, too.

This is our motivation for considering a generic Einstein-Hilbert action with a Newton constant proportional to $\varepsilon$. For the discussion in this section it is not necessary to specify the physical role of the action under consideration - the arguments apply to both bare and effective (average) actions. In both cases our aim is eventually to make sense of, and to calculate

$$
\frac{1}{\varepsilon} \int \mathrm{d}^{2+\varepsilon} x \sqrt{g} R
$$

in the limit $\varepsilon \rightarrow 0$.

(2). It turns out helpful to consider the transformation behavior of the Einstein-Hilbert action under Weyl rescalings. In this way an expansion in powers of $\varepsilon$ is more straightforward. Loosely speaking, the reason why Weyl variations are related to the $2 \mathrm{D}$ limit resides in the fact that the conformal factor is the only dynamical part of the metric that "survives" when the limit $d \rightarrow 2$ is taken, i.e. the conformal sector captures the essential information in $d=2+\varepsilon$. This idea is detailed in subsection 3.1.

Weyl transformations are defined by the pointwise rescaling

$$
g_{\mu \nu}(x)=\mathrm{e}^{2 \sigma(x)} \hat{g}_{\mu \nu}(x)
$$

with $\sigma$ a scalar function on the spacetime manifold. In appendix A we list the transformation behavior of all quantities relevant in this section.

From (3.2) it follows that $g_{\mu \nu}$ is invariant under the split-symmetry transformations

$$
\hat{g}_{\mu \nu} \rightarrow \mathrm{e}^{2 \chi} \hat{g}_{\mu \nu}, \quad \sigma \rightarrow \sigma-\chi
$$

Thus, any functional of the full metric $g_{\mu \nu}$ rewritten in terms of $\hat{g}_{\mu \nu}$ and $\sigma$ is invariant under (3.3). On the other hand, a functional of $\hat{g}_{\mu \nu}$ and $\sigma$ which is not split symmetry invariant cannot be expressed as a functional involving only $g_{\mu \nu}$, but it contains an "extra $\hat{g}_{\mu \nu}$-dependence" [23].

Before actually calculating the 2D limit of (3.1) in section 3.3 and 3.4 in a gauge invariant manner, we illustrate the situation in section 3.1 by employing the conformal gauge, and we give some general arguments in section 3.2 why and in what sense the limit is well defined. 


\subsection{Lessons from the conformal gauge}

In exactly 2 spacetime dimensions any metric $g$ can be parametrized by a diffeomorphism $f$ and a Weyl scaling $\sigma$ :

$$
f^{*} g=\mathrm{e}^{2 \sigma} \hat{g}_{\{\tau\}},
$$

where $f^{*} g$ denotes the pullback of $g$ by $f$, and $\hat{g}_{\{\tau\}}$ is a fixed reference metric that depends only on the Teichmüller parameters $\{\tau\}$ or "moduli" [30]. Stated differently, a combined Diff $\times$ Weyl transformation can bring any metric to a reference form. Thus, the moduli space is the remaining space of inequivalent metrics, $\mathcal{M}_{h}=\mathcal{G}_{h} /(\text { Diff } \times \text { Weyl })_{h}$, where $\mathcal{G}_{h}$ is the space of all metrics on a genus- $h$ manifold. ${ }^{1}$ Its precise form is irrelevant for the present discussion. Accordingly, if not needed we do not write down the dependence on $\{\tau\}$ explicitly in the following. Here we consider $\hat{g}$ a reference metric for a fixed topological sector.

In order to cope with the redundancies stemming from diffeomorphism invariance we can fix a gauge by picking one representative among the possible choices for $f$ in eq. (3.4), the most natural choice being the conformal gauge:

$$
g_{\mu \nu}=\mathrm{e}^{2 \sigma} \hat{g}_{\mu \nu} .
$$

Equation (3.5) displays very clearly the special role of 2 dimensions: the metric depends only on the conformal factor and possibly on some topological moduli parameters. Since the latter are global parameters, we see that locally the metric is determined only by the conformal factor.

(1) Conformal flatness. At this point a comment is in order. By choosing an appropriate coordinate system it is always possible to bring a $2 \mathrm{D}$ metric to the form

$$
g_{\mu \nu}=\mathrm{e}^{2 \sigma} \delta_{\mu \nu},
$$

in the neighborhood of an arbitrary spacetime point, where $\delta_{\mu \nu}$ is the flat Euclidean metric (see ref. [31] for instance). However, this is a local property only. For a general metric on a general 2D manifold there exists no scalar function $\sigma$ satisfying (3.6) globally. ${ }^{2}$ Rather must the reference metric in eq. (3.5) be compatible with all topological constraints, e.g. the value of the integral $\int \sqrt{\hat{g}} \hat{R}$ which is fixed by the Euler characteristic, a topological invariant that measures the number of handles of the manifold. As a consequence, we cannot restrict our discussion to a globally conformally flat metric in general.

\footnotetext{
${ }^{1}$ For the topology of a sphere $\mathcal{M}_{h}=\mathcal{M}_{0}$ is trivial, while for a torus there is one complex parameter, $\tau$, assuming values in the fundamental region, $F_{0}$. Apart from such simple examples it is notoriously involved to find moduli spaces [30].

${ }^{2}$ This can be understood by means of the following counterexample. Consider the standard sphere $S^{2} \subset \mathbb{R}^{3}$ with the induced metric. Upon stereographic projection the sphere is parametrized by isothermal coordinates, say $(u, v)$, where the metric assumes the form $g=\frac{4}{\left(1+u^{2}+v^{2}\right)^{2}}\left(\mathrm{~d} u^{2}+\mathrm{d} v^{2}\right)$. Setting $\sigma \equiv$ $\ln \left(\frac{2}{1+u^{2}+v^{2}}\right)$ we have $g=\mathrm{e}^{2 \sigma} \hat{g}$ with $\hat{g}=\delta$. If we assumed that $g=\mathrm{e}^{2 \sigma} \hat{g}$ holds globally for a valid scalar function $\sigma$, we could make use of identity (A.12) to arrive at a contradiction for the Euler characteristic $\chi=2$, namely: $8 \pi=4 \pi \chi \equiv \int \sqrt{g} R=\int \sqrt{\hat{g}}(\hat{R}-2 \hat{\square} \sigma)=-2 \int \sqrt{\hat{g}} \hat{\square} \sigma=0$, since $\hat{R}=0$ for the flat metric, and since the sphere has vanishing boundary. A resolution to this contradiction is to take into account that we need (at least) two coordinate patches all of which have a boundary contributing to $\int \sqrt{g} R$. Decomposing $S^{2}$ into two half spheres, $H_{+}$and $H_{-}$, for instance, and using $\hat{\square} \sigma=-4 /\left(1+u^{2}+v^{2}\right)^{2}$, we obtain $\int \sqrt{g} R=-2 \int_{H_{+}} \sqrt{\hat{g}} \hat{\square} \sigma-2 \int_{H_{-}} \sqrt{\hat{g}} \hat{\square} \sigma=8 \pi=4 \pi \chi$, as it should be.
} 
(2) Diff $\times$ Weyl invariant functionals. This has a direct impact on diffeomorphism and Weyl invariant functionals $F: g \mapsto F[g]$. The naive argument claiming that diffeomorphism invariance can be exploited to make $g_{\mu \nu}$ conformally flat, and then Weyl invariance to bring it to the form $\delta_{\mu \nu}$ such that $F[g]=F[\delta]$ would be independent of the metric, i.e. constant, is wrong actually. The global properties of the manifold thwart this argument.

When choosing appropriate local coordinates to render $g$ flat up to Weyl rescaling, there is some information of the metric implicitly encoded in the coordinate system, e.g. in the boundary of each patch, giving rise to a remaining metric dependence in $F$. A combined Diff $\times$ Weyl transformation can bring the metric to unit form, but it changes boundary conditions (like periodicity constraints for a torus) as well (see e.g. [32]). Therefore, $F$ is in fact constant with respect to local properties of the metric, while it can still depend on global parameters. According to eq. (3.4) these are precisely the moduli parameters. Hence, the metric dependence of any $2 D$ functional which is both diffeomorphism and Weyl invariant is reduced to a dependence on $\{\tau\}$, and we can write $F[g]=f(\{\tau\})$ where $f$ is a function (not a functional).

(3) Calculating 2D limits. Let us come back to the aim of this section, simplifying calculations by employing the conformal gauge (3.5). Following the previous discussion we should not rely on the choice (3.6). Nevertheless, as an example we may assume for a moment that the manifold's topology is consistent with a metric $\hat{g}$ that corresponds to a flat space, where - for the above reasons - conformal flatness is not expressed in local coordinates as in (3.6) but by the coordinate free condition $\hat{R}=0$, which is possible iff the Euler characteristic vanishes. The general case with arbitrary topologies will be covered in section 3.3. We now aim at finding a scalar function $\sigma$ which is compatible with eq. (3.5) with $g_{\mu \nu}$ given. Exploiting the identities (A.10) and (A.11) given in the appendix with $\hat{R}=0$ we obtain

$$
R=-2 \square \sigma
$$

Once we have found a solution $\sigma$ to eq. (3.7), it is clear that $\sigma^{\prime}=\sigma+($ zero modes of $\square$ ) defines a solution, too. In particular, we can subtract from $\sigma$ its projection onto the zero modes. This way, we can always obtain a solution to (3.7) which is free of zero modes. Thus, we can assume that $\sigma$ does not contain any zero modes before actually having computed it. In doing so, relation (3.7) can safely be inverted (cf. appendix A for a more detailed discussion of zero modes):

$$
\sigma=-\frac{1}{2} \square^{-1} R
$$

Note that the possibility of performing such a direct inversion is due to the simple structure of eq. (3.7) which, in turn, is a consequence of $\hat{R}=0$.

Now we leave the strictly 2-dimensional case and try to "lift" the discussion to $d=2+\varepsilon$. For this purpose we make the assumption that we can still parametrize the metric by (3.5) with a reference metric $\hat{g}$ whose associated scalar curvature vanishes, $\hat{R}=0$. (Once again, the general case will be discussed in section 3.3.) In this case, by employing equations (A.9) 
and (A.10) we obtain the following relation for the integral (3.1):

$$
\frac{1}{\varepsilon} \int \mathrm{d}^{2+\varepsilon} x \sqrt{g} R=\frac{1}{\varepsilon} \int \mathrm{d}^{2} x \sqrt{\hat{g}}[\varepsilon \sigma(-\hat{\square}) \sigma]+\mathcal{O}(\varepsilon) .
$$

This expression can be rewritten by means of the $2+\varepsilon$-dimensional analogues of eqs. (3.7) and (3.8) which read $R=-2 \square \sigma+\mathcal{O}(\varepsilon)$ and $\sigma=-\frac{1}{2} \square^{-1} R+\mathcal{O}(\varepsilon)$, respectively, and we arrive at the result

$$
\frac{1}{\varepsilon} \int \mathrm{d}^{2+\varepsilon} x \sqrt{g} R=-\frac{1}{4} \int \mathrm{d}^{2} x \sqrt{g} R \square^{-1} R+\mathcal{O}(\varepsilon) . \quad(\hat{R}=0)
$$

Clearly, the assumption $\hat{R}=0$ is quite restrictive. But already in this simple setting we make a crucial observation: the emergence of a non-local action from a purely local one in the limit $d \rightarrow 2$. More precisely, in the $2 D$ limit the Einstein-Hilbert type action $\frac{1}{\varepsilon} \int \mathrm{d}^{2+\varepsilon} x \sqrt{g} R$ becomes proportional to the induced gravity action. As we will see below, a similar result is obtained for general topologies without any assumption on $\hat{R}$.

\subsection{General properties of the limit}

(1) Existence of the limit. In the following we argue that $\lim _{\varepsilon \rightarrow 0}\left(\frac{1}{\varepsilon} \int \mathrm{d}^{2+\varepsilon} x \sqrt{g} R\right)$ is indeed a meaningful quantity without restricting ourselves to a particular topology or gauge. For convenience let us set

$$
\mathscr{S}_{\varepsilon}[g] \equiv \int \mathrm{d}^{2+\varepsilon} x \sqrt{g} R
$$

We would like to establish that $\mathscr{S}_{\varepsilon}[g]$ has a Taylor series in $\varepsilon$ whose first nonzero term which is sensitive to the local properties of $g_{\mu \nu}$ is of order $\varepsilon$.

For the proof we make use of the relation $R_{\mu \nu}=\frac{1}{2} g_{\mu \nu} R$, valid in $d=2$ for any metric, so that the Einstein tensor vanishes identically in $d=2$,

$$
\left.G_{\mu \nu}\right|_{d=2}=0
$$

Going slightly away from 2 dimensions, $d=2+\varepsilon$, we assume continuity and thus conclude that $\left.G_{\mu \nu}\right|_{d=2+\varepsilon}=\mathcal{O}(\varepsilon)$. Furthermore, the order $\varepsilon^{1}$ is really the first nonvanishing term of the Taylor series with respect to $\varepsilon$ in general, i.e. $\left.G_{\mu \nu}\right|_{d=2+\varepsilon}$ is not of order $\mathcal{O}\left(\varepsilon^{2}\right)$ or higher. This can be seen by taking the trace of $G_{\mu \nu}$,

$$
g^{\mu \nu} G_{\mu \nu}=g^{\mu \nu}\left(R_{\mu \nu}-\frac{1}{2} g_{\mu \nu} R\right)=R-\frac{d}{2} R=-\frac{1}{2} R \varepsilon .
$$

Therefore, we have $G_{\mu \nu} \propto \varepsilon$ (of course we assume $R \neq 0$ since $\mathscr{S}_{\varepsilon}$ would vanish identically otherwise), and, similarly $G^{\mu \nu} \propto \varepsilon$. But even the non-trace (tensor) parts of $G_{\mu \nu}$ can be expected to be of order $\varepsilon$ in general, as the following argument suggests. Let us consider a Weyl transformation of the metric, $g_{\mu \nu}=\mathrm{e}^{2 \sigma} \hat{g}_{\mu \nu}$. The corresponding transformation of the Einstein tensor is given by equation (A.6) in the appendix. Now, let us assume that $\hat{g}_{\mu \nu}$ 
belongs to an Einstein manifold, i.e. to a maximally symmetric spacetime. ${ }^{3}$ In this case the Ricci tensor is proportional to the scalar curvature, $\hat{R}_{\mu \nu}=\frac{1}{d} \hat{g}_{\mu \nu} \hat{R}$. Then the Einstein tensor reads

$$
G_{\mu \nu}=(d-2)\left[-\frac{1}{2 d} \hat{g}_{\mu \nu} \hat{R}-\hat{D}_{\mu} \hat{D}_{\nu} \sigma+\hat{g}_{\mu \nu} \hat{\square} \sigma+\hat{D}_{\mu} \sigma \hat{D}_{\nu} \sigma+\frac{d-3}{2} \hat{g}_{\mu \nu} \hat{D}_{\alpha} \sigma \hat{D}^{\alpha} \sigma\right],
$$

so we find $G_{\mu \nu} \propto \varepsilon$ again.

This $\varepsilon$-proportionality is exploited now to make a statement about the Taylor series of $\mathscr{S}_{\varepsilon}$. For that purpose we consider the variation of $\mathscr{S}_{\varepsilon}$ with respect to $g_{\mu \nu}$ (assuming vanishing surface terms):

$$
\frac{\delta \mathscr{S}_{\varepsilon}[g]}{\delta g_{\mu \nu}(x)}=\int \mathrm{d}^{2+\varepsilon} y \sqrt{g}\left[\frac{1}{2} g^{\mu \nu} R-R^{\mu \nu}\right] \delta(x-y)=-\sqrt{g} G^{\mu \nu}=\mathcal{O}(\varepsilon) .
$$

As a result we obtain $\mathscr{S}_{\varepsilon}[g]=C+\mathcal{O}(\varepsilon)$, where the constant $C$ is independent of $g_{\mu \nu}$. Clearly, $C$ is obtained by computing $\mathscr{S}_{\varepsilon}$ in $d=2$, which is known to lead to the Euler characteristic $\chi$ :

$$
C=\left.\mathscr{S}_{\varepsilon}\right|_{\varepsilon=0}=4 \pi \chi
$$

That is, we have $\mathscr{S}_{\varepsilon}=4 \pi \chi+\mathcal{O}(\varepsilon)$. (This result differs from ref. [39], but it is in agreement with refs. [40-42]). As a consequence, the integral (3.1) amounts to

$$
\frac{1}{\varepsilon} \int \mathrm{d}^{2+\varepsilon} x \sqrt{g} R=\frac{4 \pi \chi}{\varepsilon}+\text { finite }=\text { top. }+ \text { finite },
$$

where 'top.' is a field independent (up to topological information) and thus irrelevant contribution to the action. The terms in (3.17) that contain the interesting information about the dynamics of the field are of order $\mathcal{O}\left(\varepsilon^{0}\right)$, so the "relevant" part of $\frac{1}{\varepsilon} \int \mathrm{d}^{2+\varepsilon} x \sqrt{g} R$ has indeed a meaningful limit $\varepsilon \rightarrow 0$.

(2) The role of the volume form. Next we argue that the important part of the $\varepsilon$ dependence of $\mathscr{S}_{\varepsilon}$ originates from the scalar density $\sqrt{g} R$ in the integrand of (3.11) alone, i.e. loosely speaking it is sufficient to employ the a priori undefined fractional integration element $\mathrm{d}^{2+\varepsilon} x$ at $\varepsilon=0$. Stated differently, all consistent definitions of " $\mathrm{d}^{2+\varepsilon} x$ " away from $\varepsilon=0$ that one might come up with are equivalent. The reason for that is the following.

Any integration over a scalar function on a manifold involves a volume form, i.e. a nowhere vanishing $d$-form (or a density in the non-orientable case), in order to define a measure. This volume form is given by $\mathrm{d}^{d} x \sqrt{g}$, where $\sqrt{g}$ is the square root of the corresponding Gramian determinant. If an integral is to be evaluated, the unit vectors of the underlying coordinate system are inserted into the volume form. Since, for any $d$, these unit vectors produce a factor of 1 when inserted into $\mathrm{d}^{d} x$, we see that it is the

\footnotetext{
${ }^{3}$ In $d>2$ it is always possible to find $\sigma$ for a given metric $g_{\mu \nu}$ such that $\hat{g}_{\mu \nu}=\mathrm{e}^{-2 \sigma} g_{\mu \nu}$ leads to a space with constant curvature provided that the manifold is compact. This is known as the Yamabe problem [3337] (while the case $d=2$ is covered by Poincaré's uniformization theorem). However, this statement does not imply that the manifold is Einstein. In fact, there are known examples of metrics which are not conformal to any Einstein metric [38]. On the other hand, in $d=2$ any Riemannian manifold is of Einstein type.
} 
remaining part of the volume element that contains its complete $d$-dependence, namely $\sqrt{g}$. In particular, $\sqrt{g}$ carries the canonical dimension of the volume element. ${ }^{4}$

To summarize, for the evaluation of $\lim _{\varepsilon \rightarrow 0} \frac{1}{\varepsilon} \mathscr{S}_{\varepsilon}$ it is sufficient to consider the $\varepsilon$-dependence of $\sqrt{g} R$, while the integration can be seen as an integration over $\mathrm{d}^{2} x$. This prescription can be considered our definition for taking the $\varepsilon$-limit in a well behaved way. Clearly, the details of the domain of integration contribute some $\varepsilon$-dependence, too. However, as we have seen in (1) in equation (3.15), the first relevant non-constant, i.e. metric dependent, part of the action comes from $\sqrt{g} R$ alone, and any further $\varepsilon$-dependent contributions would be of order $\varepsilon^{2}$. This makes clear that our argument is valid in the special case of an integral over $\sqrt{g} R$, but not for arbitrary integrands.

(3) Comment and comparison with related work. As an aside we note that in ref. [40] it is argued that the irrelevant divergent term in (3.17) can be made vanish by subtracting the term $\frac{1}{\varepsilon} \int \mathrm{d}^{d} x \sqrt{\tilde{g}} \tilde{R}$ from $\frac{1}{\varepsilon} \int \mathrm{d}^{d} x \sqrt{g} R$ where the metric $\tilde{g}_{\mu \nu}$ is assumed to be $g_{\mu \nu^{-}}$ dependent but chosen in such a way that the resulting field equations for $g_{\mu \nu}$ do not change when $d$ approaches 2 . That means, the $g_{\mu \nu}$-variation of the subtracted term (and, in turn its variation w.r.t. $\tilde{g}$ ) must vanish for $d \rightarrow 2$, leading to the requirement $\lim _{\varepsilon \rightarrow 0}\left(\frac{1}{\varepsilon} \tilde{G}_{\mu \nu}\right)=0$ for the corresponding Einstein tensor. This subtraction term would cancel the $\varepsilon$-pole in (3.17). It is assumed in [40] that such a term exists for some metric $\tilde{g}_{\mu \nu}$ which is conformally related to $g_{\mu \nu}$. However, it remains unclear if this is possible at all. According to the above argument in (1) we would rather expect $\frac{1}{\varepsilon} \tilde{G}_{\mu \nu}$ to remain finite in the limit $\varepsilon \rightarrow 0$.

Unlike ref. [40], we do not need to subtract further $g_{\mu \nu}$-dependent terms from the action here, and our discussion is valid for all metrics.

\subsection{Establishing the 2D limit}

Next we determine the first relevant order of the Taylor series, providing the basis for our main statements. Let us define the $\varepsilon$-dependent action functional

$$
Y_{\varepsilon}[g] \equiv \frac{1}{\varepsilon} \int \mathrm{d}^{2+\varepsilon} x \sqrt{g} R-\frac{4 \pi \chi}{\varepsilon} .
$$

Here $\chi$ denotes again the metric independent Euler characteristic defined in strictly 2 dimensions. Corresponding to the arguments of section $3.2, Y_{\varepsilon}$ is well defined in the limit $\varepsilon \rightarrow 0$ since it is of order $\varepsilon^{0}$. Therefore, $Y[g]$ defined by

$$
Y[g] \equiv \lim _{\varepsilon \rightarrow 0} Y_{\varepsilon}[g]
$$

is a finite functional.

To expand the integral in (3.18) in powers of $\varepsilon$ we make use of the general transformation law of $\int \mathrm{d}^{d} x \sqrt{g} R$ under Weyl rescalings $g_{\mu \nu}=\mathrm{e}^{2 \sigma} \hat{g}_{\mu \nu}$, given by equation (A.9) in

\footnotetext{
${ }^{4}$ Our conventions for the canonical mass dimensions are such that all coordinates are dimensionless, $\left[x^{\mu}\right]=0$, while the metric components have $\left[g_{\mu \nu}\right]=-2$, giving $\mathrm{d} s^{2}=g_{\mu \nu} \mathrm{d} x^{\mu} \mathrm{d} x^{\nu}$ the canonical dimension of an area, $\left[\mathrm{d} s^{2}\right]=-2$, whatever is the value of $d$. Hence $\left[\mathrm{d} x^{\mu}\right]=0$ and $[\sqrt{g}]=-d$.

As a consequence, the symbolic integration over the remaining "fraction of a dimension", $\mathrm{d}^{\varepsilon} x$, is irrelevant even for the dimension of $\mathscr{S}_{\varepsilon}[g]$.
} 
the appendix. This yields

$$
\begin{aligned}
Y_{\varepsilon}[g] & =\frac{1}{\varepsilon} \int \mathrm{d}^{2+\varepsilon} x \sqrt{\hat{g}} \mathrm{e}^{\varepsilon \sigma}\left[\hat{R}+(1+\varepsilon) \varepsilon\left(\hat{D}_{\mu} \sigma\right)\left(\hat{D}^{\mu} \sigma\right)\right]-\frac{4 \pi \chi}{\varepsilon} \\
& =\frac{1}{\varepsilon} \int \mathrm{d}^{2+\varepsilon} x \sqrt{\hat{g}} \hat{R}-\frac{4 \pi \chi}{\varepsilon}+\int \mathrm{d}^{2} x \sqrt{\hat{g}}\left(\hat{R} \sigma+\hat{D}_{\mu} \sigma \hat{D}^{\mu} \sigma\right)+\mathcal{O}(\varepsilon) .
\end{aligned}
$$

We observe that the first two terms of the second line of (3.20) can be combined into $Y_{\varepsilon}[\hat{g}]$. Furthermore, the terms involving the parameter of the Weyl transformation, $\sigma$, are seen to agree with the definition in (2.23) and can be written as $\int \mathrm{d}^{2} x \sqrt{\hat{g}}\left[\hat{D}_{\mu} \sigma \hat{D}^{\mu} \sigma+\hat{R} \sigma\right] \equiv$ $2 \Delta I[\sigma ; \hat{g}]$. This, in turn, can be expressed by means of the (normalized) induced gravity functional [27], defined by ${ }^{5}$

$$
I[g] \equiv \int \mathrm{d}^{2} x \sqrt{g} R \square^{-1} R .
$$

As shown in appendix A, the change of $I$ under a finite Weyl transformation of the metric in its argument equals precisely $-8 \Delta I$ which therefore has the interpretation of a WessZumino term, a 1-cocycle related to the Abelian group of Weyl transformations [43]: ${ }^{6}$

$$
I\left[\mathrm{e}^{2 \sigma} \hat{g}\right]-I[\hat{g}]=-8 \Delta I[\sigma ; \hat{g}]
$$

Inserting (3.22) into (3.20) leads to

$$
Y_{\varepsilon}[g]=Y_{\varepsilon}[\hat{g}]+2 \Delta I[\sigma ; \hat{g}]+\mathcal{O}(\varepsilon)=Y_{\varepsilon}[\hat{g}]+\frac{1}{4} I[\hat{g}]-\frac{1}{4} I[g]+\mathcal{O}(\varepsilon) .
$$

Rearranging terms and taking the limit $\varepsilon \rightarrow 0$ results in the important identity

$$
Y[g]+\frac{1}{4} I[g]=Y[\hat{g}]+\frac{1}{4} I[\hat{g}]
$$

Note that the 1.h.s. of eq. (3.24) depends on the full metric $g=\mathrm{e}^{2 \sigma} \hat{g}$ while the r.h.s. depends on $\hat{g}$ only.

For the further analysis it is convenient to introduce the functional

$$
F[g] \equiv Y[g]+\frac{1}{4} I[g]
$$

By construction $F$ has the following properties:

(i). It is diffeomorphism invariant since it has been constructed from diffeomorphism invariant objects only.

(ii). It is a functional in $d=2$ precisely since the $\varepsilon$-limit has already been taken.

\footnotetext{
${ }^{5}$ If the scalar Laplacian $\square$ has zero modes, then $\square^{-1}$ is defined as the inverse of $\square$ on the orthogonal complement to its kernel, that is, before $\square^{-1}$ acts on a function it implicitly projects onto nonzero modes. For the arguments presented in this section we may assume that $\square$ does not have any zero modes, although a careful analysis shows that the inclusion of zero modes does not change our main results (see detailed discussion in appendix A, in particular section A.2).

${ }^{6}$ As a consequence of identity (3.22), the Liouville action (2.22) can be rewritten as $\Gamma_{k}^{\mathrm{L}}[\phi ; \hat{g}]=\frac{a_{1}}{4} I\left[\mathrm{e}^{2 \phi} \hat{g}\right]+$ $\frac{1}{2} a_{1} a_{2} \int \mathrm{d}^{2} x \sqrt{\operatorname{det}\left(\mathrm{e}^{2 \phi} \hat{g}\right)}-\frac{a_{1}}{4} I[\hat{g}]$. Note that the first two terms on the r.h.s. of this equation depend on $\phi$ and $\hat{g}_{\mu \nu}$ only in the combination $\mathrm{e}^{2 \phi} \hat{g}_{\mu \nu}=g_{\mu \nu}$.
} 
(iii). It is insensitive to the conformal factor of its argument since from eq. (3.24) follows Weyl invariance:

$$
F\left[\mathrm{e}^{2 \sigma} \hat{g}\right]=F[\hat{g}] .
$$

Thanks to our preparations in section 3.1 we can conclude immediately that $F$ is constant apart from a remaining dependence on some moduli $\{\tau\}$ possibly. Here it is crucial that the moduli are global parameters of purely topological origin. They are insensitive to the local properties of the metric, in particular they do not depend on a spacetime point. These arguments show that the functional $F[g]$ becomes a function of the moduli, say $C(\{\tau\})$. The precise dependence of $F$ on these moduli is irrelevant for the present discussion since they encode only topological information. We thus have

$$
F[g]=C(\{\tau\})
$$

i.e. $F$ is a metric independent constant functional, up to topological terms.

For the functional $Y[g]$ defined in eq. (3.19) we obtain, using eq. (3.25),

$$
Y[g]=-\frac{1}{4} I[g]+C(\{\tau\})
$$

which leads to our final result:

$$
\frac{1}{\varepsilon} \int \mathrm{d}^{2+\varepsilon} x \sqrt{g} R=-\frac{1}{4} \int \mathrm{d}^{2} x \sqrt{g} R \square^{-1} R+\frac{4 \pi \chi}{\varepsilon}+C(\{\tau\})+\mathcal{O}(\varepsilon) .
$$

The terms $4 \pi \chi / \varepsilon$ and $C(\{\tau\})$ are topology dependent but independent of the local properties of the metric, and thus they may be considered irrelevant for most purposes.

So we have established that the limit $d \rightarrow 2$ of the Einstein-Hilbert action equals precisely the induced gravity action up to topological terms. Clearly the most remarkable aspect of this limiting procedure is that it leads from a local to a non-local action.

A similar mechanism has been discussed earlier in the framework of dimensional regularization [43]. The result (3.29) is in agreement with the one of reference [41] where it has been obtained by means of a different reasoning based on the introduction of a Weyl gauge potential.

\subsection{The full Einstein-Hilbert action for $\varepsilon \rightarrow 0$}

Including also the cosmological constant term, the Einstein-Hilbert truncation of the (gravitational part of the) effective average action reads

$$
\Gamma_{k}^{\text {grav }}[g]=\frac{1}{16 \pi G_{k}} \int \mathrm{d}^{d} x \sqrt{g}\left(-R+2 \Lambda_{k}\right),
$$

with the dimensionful Newton and cosmological constant, $G_{k}$ and $\Lambda_{k}$, respectively.

(1). As we have mentioned already, RG studies in $d=2+\varepsilon$ show that the $\beta$-functions of the dimensionless versions of these couplings, $g_{k} \equiv k^{d-2} G_{k}$ and $\lambda_{k} \equiv k^{-2} \Lambda_{k}$, possess a nontrivial fixed point which is proportional to $\varepsilon$ [8] (see also [1, 22, 24-26, 44-58]),

$$
g_{*} \propto \varepsilon \quad \text { and } \quad \lambda_{*} \propto \varepsilon .
$$


Thus, at least in the vicinity of this non-Gaussian fixed point the dimensionful couplings are of the form

$$
G_{k} \equiv \varepsilon \breve{G}_{k}, \quad \Lambda_{k} \equiv \varepsilon \breve{\Lambda}_{k},
$$

where $\breve{G}_{k}$ and $\breve{\Lambda}_{k}$ are of order $\mathcal{O}\left(\varepsilon^{0}\right)$. Making use of eq. (3.29) in the limit $\varepsilon \rightarrow 0$ we arrive at the 2-dimensional effective average action

$$
\Gamma_{k}^{\text {grav }, 2 \mathrm{D}}[g]=\frac{1}{64 \pi \breve{G}_{k}} \int \mathrm{d}^{2} x \sqrt{g} R \square^{-1} R+\frac{\breve{\Lambda}_{k}}{8 \pi \breve{G}_{k}} \int \mathrm{d}^{2} x \sqrt{g}+\text { top. }
$$

Here 'top' refers again to topology dependent terms which are insensitive to the local properties of the metric. The result (3.33) is quite general; it holds for any RG trajectory provided that the couplings $G_{k}$ and $\Lambda_{k}$ in $d=2+\varepsilon$ are of first order in $\varepsilon$.

As an aside we note that the topological terms in (3.33) include a contribution proportional to $\int \sqrt{g} R=4 \pi \chi$. Thus, eq. (3.33) contains the induced gravity action, a cosmological constant term, and the $\chi$-term. These are precisely the terms that were included in the truncation ansatz in ref. [22]. By contrast, in our approach they are not put in by hand through an ansatz, but they rather emerge as a result from the Einstein-Hilbert action in the 2D limit.

(2). If we want to consider $\Gamma_{k}$ exactly at the NGFP, we can insert the known fixed point values, where the one of Newton's constant is given by $g_{*}=\varepsilon / b$ according to eq. (1.5). The coefficient $b$ is independent of the cutoff scheme underlying the computation. It depends, however, on the parametrization of the metric. In the linear parametrization, $g_{\mu \nu}=\bar{g}_{\mu \nu}+$ $h_{\mu \nu}$, it is given by $[1,8,22,24-26,44,45,48,49]^{7}$

$$
b=\frac{2}{3}(19-N),
$$

while the exponential parametrization [59], $g_{\mu \nu}=\bar{g}_{\mu \rho}\left(\mathrm{e}^{h}\right)^{\rho}{ }_{\nu}$, leads to $[22,26,50-58]$

$$
b=\frac{2}{3}(25-N) .
$$

While we will argue in section 5 that the two parametrizations might possibly describe different universality classes, to make contact to the results known from 2-dimensional conformal field theory the exponential parametrization turns out to be more appropriate. Therefore, we will mostly state the results based on eq. (3.35) in the following, although the analogues for the linear parametrization can be obtained simply by replacing $25 \rightarrow 19$. Using the definition (3.21) and combining (3.33) with (3.35) we obtain the NGFP action

$$
\Gamma_{k}^{\text {grav }, 2 \mathrm{D}, \mathrm{NGFP}}[g]=\frac{(25-N)}{96 \pi} I[g]+\frac{(25-N)}{12 \pi} k^{2} \breve{\lambda}_{*} \int \mathrm{d}^{2} x \sqrt{g}+\text { top },
$$

where $\breve{\lambda}_{*} \equiv \lambda_{*} / \varepsilon$ is cutoff dependent and thus left unspecified here. The actions (3.33) and (3.36) will be the subject of our discussion in section 4 .

\footnotetext{
${ }^{7}$ When the running of the Gibbons-Hawking surface term instead of the pure Einstein-Hilbert action is computed, the result reads $b=\frac{2}{3}(1-N)[46,47]$. See ref. [24, 25] for a discussion.
} 
(3). Finally, let us briefly establish the connection with Liouville theory. For this purpose we separate the conformal factor from the rest of the metric. Inserting

$$
g_{\mu \nu}=\mathrm{e}^{2 \phi} \hat{g}_{\mu \nu}
$$

into eq. (3.33) for $\Gamma_{k}^{\text {grav }, 2 \mathrm{D}}[g]$ and using (A.21) and (A.22) from the appendix yields

$$
\begin{aligned}
\Gamma_{k}^{\text {grav }, 2 \mathrm{D}}[\phi ; \hat{g}]= & \frac{1}{64 \pi \breve{G}_{k}} \int \mathrm{d}^{2} x \sqrt{\hat{g}} \hat{R} \hat{\square}^{-1} \hat{R} \\
& -\frac{1}{16 \pi \breve{G}_{k}} \int \mathrm{d}^{2} x \sqrt{\hat{g}}\left[\hat{D}_{\mu} \phi \hat{D}^{\mu} \phi+\hat{R} \phi-2 \breve{\Lambda}_{k} \mathrm{e}^{2 \phi}\right]+\text { top }
\end{aligned}
$$

where $\hat{g}_{\mu \nu}$ is a fixed reference metric for the topological sector (i.e. a point in moduli space) under consideration. Hence, the effective average action for the conformal factor in precisely 2 dimensions is nothing but the Liouville action.

Of course, this is well known to happen if one starts from the induced gravity action, an object that lives already in 2D. It is quite remarkable and nontrivial, however, that Liouville theory can be regarded the limit of the higher dimensional Einstein-Hilbert theory. Note that this result is consistent with the discussions in refs. [40, 42] (cf. also [60]).

\subsection{Aside: is there a generalization to $4 \mathrm{D}$ ?}

For the sake of completeness we would like to comment on a generalization of our results to 4 dimensions. At first sight there seems to be a remarkable similarity. Dimensional analysis suggests that the role of the $R$-term in the Einstein-Hilbert action near 2 dimensions is now played by curvature-square terms in $d=4+\varepsilon$. The gravitational part of the action assumes the form $\Gamma_{k}[g]=\Gamma_{k}^{\mathrm{EH}}+\int \mathrm{d}^{4+\varepsilon} x \sqrt{g}\left\{\frac{1}{a_{k}} E+\frac{1}{b_{k}} F+\frac{1}{c_{k}} R^{2}\right\}$ where $F \equiv C_{\mu \nu \rho \sigma} C^{\mu \nu \rho \sigma}$ is the square of the Weyl tensor, and $E \equiv R_{\mu \nu \rho \sigma} R^{\mu \nu \rho \sigma}-4 R_{\mu \nu} R^{\mu \nu}+R^{2}+\frac{d-4}{18} R^{2}$ gives rise to the Gauss-Bonnet-Euler topological invariant when integrated over in $d=4$. Considerations of nontrivial cocycles of the Weyl group show that the corresponding Wess-Zumino action in $d=4$ is generated by the $E$ - and the $F$-term [43], analogous to the generation of $\Delta I$ in section 3.3 due to the $R$-term. It may thus be expected that there would be a mechanism to take the $4 \mathrm{D}$ limit, similar to the one of section 3.3 but now for $E$ and $F$ instead of $R$, if the couplings $a_{k}$ and $b_{k}$ were of first order in $\varepsilon$.

At one-loop level the $\beta$-functions in $d=4+\varepsilon$ have indeed a fixed point with $a_{*}=\mathcal{O}(\varepsilon)$, $b_{*}=\mathcal{O}(\varepsilon)$ and $c_{*}$ finite [61]. There are, however, two crucial differences in comparison with the 2-dimensional case: (i) The term $\int \mathrm{d}^{4} x \sqrt{g} F$ is not a topological invariant, i.e. there is no appropriate subtraction analogous to definition (3.18), and the limit $\varepsilon \rightarrow 0$ remains problematic. (ii) Even if we managed to define some 4D-functional similar to (3.25) which is both diffeomorphism and Weyl invariant, this would not be sufficient to conclude that the functional is constant since in $d=4$ the space of metrics modulo Diff $\times$ Weyl-transformation is too large and cannot be classified in terms of topological parameters. Roughly speaking, if we found a way to circumvent problem (i), the 4D limit of the above action computed with our methods might lead to the same non-local action as found in [43], but this would not represent the general $4 \mathrm{D}$ limit since the latter must certainly contain further terms that 
do not originate from a variation of the conformal factor alone. In summary, in spite of many similarities to the $2 \mathrm{D}$ case there seems to be no direct generalization of our approach of computing a non-local limit action to 4 spacetime dimensions. Nevertheless, we expect that the 4D fixed point action contains non-local terms, too.

\section{The NGFP as a conformal field theory}

We can summarize the previous sections by saying that every trajectory $k \mapsto\left(g_{k}, \lambda_{k}\right) \equiv$ $\left(\breve{g}_{k}, \breve{\lambda}_{k}\right) \varepsilon$, i.e. every solution to the RG equations of the Einstein-Hilbert truncation in $2+\varepsilon$ dimensions, induces the following intrinsically 2-dimensional running action:

$$
\Gamma_{k}^{\mathrm{grav}, 2 \mathrm{D}}[g]=\frac{1}{96 \pi}\left(\frac{3}{2} \frac{1}{\breve{g}_{k}}\right)\left[I[g]+8 \breve{\lambda}_{k} k^{2} \int \mathrm{d}^{2} x \sqrt{g}\right] .
$$

In this section we discuss the main properties of this RG trajectory, in particular its fixed point.

(1) The fixed point functional. Strictly speaking, the theory space under consideration comprises functionals which depend on the dimensionless metric $\tilde{g}_{\mu \nu} \equiv k^{2} g_{\mu \nu}$. For any average action $\Gamma_{k}[g]$ we define its analog in the dimensionless setting by $\mathcal{A}_{k}[\tilde{g}] \equiv \Gamma_{k}\left[\tilde{g} k^{2}\right]$. Thus, equation (4.1) translates into

$$
\mathcal{A}_{k}[\tilde{g}]=\frac{1}{96 \pi}\left(\frac{3}{2} \frac{1}{\breve{g}_{k}}\right)\left[I[\tilde{g}]+8 \breve{\lambda}_{k} \int \mathrm{d}^{2} x \sqrt{\tilde{g}}\right] .
$$

It is this functional that becomes strictly constant at the NGFP: $\mathcal{A}_{k} \rightarrow \mathcal{A}_{*}$, with

$$
\mathcal{A}_{*}[\tilde{g}]=\frac{1}{96 \pi}\left(\frac{3}{2} \frac{1}{\breve{g}_{*}}\right)\left[I[\tilde{g}]+8 \breve{\lambda}_{*} \int \mathrm{d}^{2} x \sqrt{\tilde{g}}\right] .
$$

For the exponential field parametrization we find the fixed point functional

$$
\mathcal{A}_{*}[\tilde{g}]=\frac{(25-N)}{96 \pi} \int \mathrm{d}^{2} x \sqrt{\tilde{g}}\left(\tilde{R} \tilde{\square}^{-1} \tilde{R}+8 \breve{\lambda}_{*}\right) .
$$

Here and in the following we usually present the results for the exponential parametrization. The corresponding formulae for the linear parametrization can be obtained by replacing $(25-N) \rightarrow(19-N)$. See also section 5 for a discussion of the different metric parametrizations.

While the NGFP is really a point in the space of $\mathcal{A}$-functionals, it is an entire line, parametrized by $k$, in the more familiar dimensionful language of the $\Gamma_{k}$ 's. Let us refer to the constant map $k \mapsto\left(g_{*}, \lambda_{*}\right) \forall k \in[0, \infty)$ as the "FP trajectory". Moving on this trajectory, the system is never driven away from the fixed point. According to eq. (3.36), it is described by the following EAA:

$$
\Gamma_{k}^{\text {grav }, 2 \mathrm{D}, \mathrm{NGFP}}[g]=\frac{(25-N)}{96 \pi}\left[I[g]+8 \breve{\lambda}_{*} k^{2} \int \mathrm{d}^{2} x \sqrt{g}\right] .
$$

As always in the EAA framework, the EAA at $k=0$ equals the standard effective action, $\Gamma=\lim _{k \rightarrow 0} \Gamma_{k}$. So, letting $k=0$ in (4.5), we conclude that the ordinary effective action related to the FP trajectory has vanishing "renormalized" cosmological constant and reads

$$
\Gamma^{\text {grav }, 2 \mathrm{D}, \mathrm{NGFP}}[g]=\frac{(25-N)}{96 \pi} \int \mathrm{d}^{2} x \sqrt{g} R \square^{-1} R .
$$


(2) The 2D stress tensor. Differentiating $\Gamma_{k}^{\text {grav,2D }}$ of equation (4.1) with respect to the metric leads to the following energy-momentum tensor in the gravitational sector [62]:

$$
\begin{array}{r}
T_{\mu \nu}^{\text {grav }}[g]=\frac{1}{96 \pi}\left(\frac{3}{2} \frac{1}{\breve{g}_{k}}\right)\left[g_{\mu \nu} D_{\rho}\left(\square^{-1} R\right) D^{\rho}\left(\square^{-1} R\right)-2 D_{\mu}\left(\square^{-1} R\right) D_{\nu}\left(\square^{-1} R\right)\right. \\
\left.+4 D_{\mu} D_{\nu}\left(\square^{-1} R\right)-4 g_{\mu \nu} R+8 \breve{\lambda}_{k} k^{2} g_{\mu \nu}\right] .
\end{array}
$$

It is easy to see that taking the trace of this tensor yields $\Theta_{k}[g]=\left(\frac{3}{2} \frac{1}{\breve{g}_{k}}\right) \frac{1}{24 \pi}\left[-R+4 \breve{\lambda}_{k} k^{2}\right]$ which, as it should be, agrees with the result from the Einstein-Hilbert action in $d>2$, see equations (2.13) and (2.15). ${ }^{8}$ As for the non-trace parts of $T_{\mu \nu}^{\text {grav }}$, the comparatively complicated non-local structures in (4.7) can be seen as the 2D replacement of the Einstein tensor in (2.11).

In absence of matter $\left(\Gamma_{k}^{\mathrm{M}}=0\right)$ the tadpole equation (2.10) boils down to $T_{\mu \nu}^{\mathrm{grav}}\left[\bar{g}_{k}^{\mathrm{sc}}\right]=0$ with the above stress tensor. Hence, self-consistent backgrounds have a constant (but $k$-dependent) Ricci scalar:

$$
\Theta_{k}\left[\bar{g}_{k}^{\mathrm{sc}}\right]=0 \quad \Leftrightarrow \quad R\left(\bar{g}_{k}^{\mathrm{sc}}\right)=4 \breve{\lambda}_{k} k^{2} .
$$

In terms of the dimensionless metric, $R\left(\tilde{\bar{g}}_{k}^{\mathrm{sc}}\right)=4 \breve{\lambda}_{k}$, in this case.

(3) Intermezzo on induced gravity. As a preparation for the subsequent discussion consider an arbitrary conformal field theory on flat Euclidean space, having central charge $c_{\mathscr{S}}$, and couple this theory to a gravitational background field $g_{\mu \nu}$, comprised in an action functional $\mathscr{S}[g]$. Then the resulting (symmetric, conserved) stress tensor,

$$
T^{(\mathscr{S})}[g]^{\mu \nu} \equiv \frac{2}{\sqrt{g}} \frac{\delta \mathscr{S}[g]}{\delta g_{\mu \nu}},
$$

will acquire a nonzero trace in curved spacetimes, of the form

$$
g_{\mu \nu} T^{(\mathscr{S})}[g]^{\mu \nu}=-c_{\mathscr{S}} \frac{1}{24 \pi} R+\text { const },
$$

where "const" is due to a cosmological constant possibly.

(3a). Above $\mathscr{S}[g]$ can stand for either a classical or an effective action. In the first case, $\mathscr{S}[g]$ might result from a CFT of fields $\chi^{I}$ governed by an action $S[\chi, g]$ upon solving the equations of motion for $\chi$, and substituting the solution $\chi_{\text {sol }}(g)$ back into the action: $\mathscr{S}[g]=S\left[\chi_{\mathrm{sol}}(g), g\right]$. If $c_{\mathscr{S}} \neq 0$ then the system displays a "classical anomaly", and Liouville theory is the prime example [63-66].

In the "effective" case, $\mathscr{S}[g]$ could be the induced gravity action $S_{\text {ind }}[g]$ which we obtain from $S[\chi, g]$ by integrating out the fields $\chi^{I}$ quantum mechanically:

$$
\mathrm{e}^{-S_{\text {ind }}[g]}=\int \mathcal{D} \chi^{I} \mathrm{e}^{-S[\chi, g]}
$$

\footnotetext{
${ }^{8}$ Note that in string theory or conformal field theory one would usually redefine the stress tensor and employ $T_{\mu \nu}^{\prime} \equiv T_{\mu \nu}-\frac{1}{2} g_{\mu \nu} \Theta$ which is traceless at the expense of not being conserved. It is the modes of $T_{\mu \nu}^{\prime}$ that satisfy a Virasoro algebra whose central extension keeps track of the anomaly coefficient then.
} 
Then $S_{\text {ind }}$ is proportional to the central charge $c_{\mathscr{S}}$,

$$
S_{\text {ind }}[g]=+\frac{c_{\mathscr{S}}}{96 \pi} I[g]+\cdots,
$$

and by (4.9) the action $S_{\text {ind }}$ gives rise to a stress tensor whose trace is precisely of the form (4.10). (The dots represent a cosmological constant term.)

(3b). It is important to observe that the functional $I[g]$ is negative, i.e. for any metric $g$ we have $\int \mathrm{d}^{2} x \sqrt{g} R \square^{-1} R<0$. (Recall that $\square^{-1}$ acts only on nonzero modes while it "projects away" the zero modes. Since $-\square$ is non-negative, we conclude that $-\square^{-1}$ has a strictly positive spectrum.) Leaving the cosmological constant term in (4.12) aside, this entails that for a positive central charge $c_{\mathscr{S}}>0$ the (non-cosmological part of the) induced gravity action is negative, $S_{\text {ind }}[g]<0$.

The implications are particularly obvious in the conformal parametrization $g=\mathrm{e}^{2 \phi} \hat{g}$, yielding

$$
S_{\text {ind }}[\phi ; \hat{g}]=-\frac{c_{\mathscr{S}}}{24 \pi} \int \mathrm{d}^{2} x \sqrt{\hat{g}}\left(\hat{D}_{\mu} \phi \hat{D}^{\mu} \phi+\hat{R} \phi\right)+\frac{c_{\mathscr{S}}}{96 \pi} I[\hat{g}]+\cdots .
$$

When $c_{\mathscr{S}}$ is positive, the field $\phi$ is unstable, it has a "wrong sign" kinetic term. Stated differently, integrating out unitary conformal matter induces an unstable conformal factor of the emergent spacetime metric.

The 4D Einstein-Hilbert action is well known to suffer from the same conformal factor instability, that is, a negative kinetic term for $\phi$ if the overall prefactor of $\int \sqrt{g} R$ is adjusted in such a way the concomitant kinetic term for the transverse-traceless (TT) metric fluctuations comes out positive, as this befits propagating physical modes. Irrespective of all questions about the conventions in which the equations are written down, the crucial signs are always such that

$$
c_{\mathscr{S}}>0 \stackrel{d=2}{\Longleftrightarrow} \phi \text { unstable } \stackrel{d>3}{\Longleftrightarrow} h_{\mu \nu}^{\mathrm{TT}} \text { stable. }
$$

We shall come back to this point in a moment.

(4) Central charge of the NGFP. The fixed point action $\mathcal{A}_{*}^{\text {grav,2D }}$ given by (4.3) describes a conformal field theory with central charge

$$
c_{\mathrm{grav}}^{\mathrm{NGF}}= \begin{cases}25-N, & \text { exponential parametrization } \\ 19-N, & \text { linear parametrization. }\end{cases}
$$

This follows by observing that for the two field parametrizations, directly at the NGFP, the trace of the stress tensor is given by

$$
\Theta_{k}[g]=\frac{1}{24 \pi}\left(-R+4 \breve{\lambda}_{*} k^{2}\right) \times \begin{cases}25-N & \text { (exp.) } \\ 19-N & \text { (lin.) } .\end{cases}
$$

Applying the rule (4.10) to eq. (4.16), we see indeed that, first, the fixed point theory is a CFT, and second, its central charge is given by $(4.15){ }^{9}$

\footnotetext{
${ }^{9}$ Reading off the central charge according to $(4.10)$ and $(4.12)$ is consistent with refs. [22, 67] where the relation between the central charge and the $\beta$-function of Newton's constant is discussed in the FRG framework, implying a relation between $c_{\mathrm{grav}}^{\mathrm{NGFP}}$ and $g_{*}$.
} 
According to eq. (4.5), the EAA related to the FP trajectory, $\Gamma_{k}^{\text {grav,2D,NGFP }}$, happens to have exactly the structure of the induced gravity action (4.12) with the corresponding central charge, for all values of the scale parameter.

At the $k=0$ endpoint of this trajectory, the dimensionful cosmological constant $\breve{\Lambda}_{k}=$ $\breve{\lambda}_{*} k^{2}$ runs to zero without any further ado, and $\Gamma_{k \rightarrow 0}^{\text {grav,2D,NGFP }}$ becomes the standard effective action (4.6). At this endpoint, by eq. (4.8), self-consistent backgrounds have vanishing curvature in the absence of matter: $R\left(\bar{g}_{k=0}^{\mathrm{sc}}\right)=0$. Therefore, we have indeed inferred a central charge pertaining to flat space by comparing (4.16) to (4.10).

(5) Auxiliary "matter" CFTs. Since the 2D gravitational fixed point action is of the induced gravity type, we can, if we wish to, introduce a conformal matter field theory which induces it when the fluctuations of those auxiliary matter degrees of freedom are integrated out (although such auxiliary fields are not required by our formalism). Denoting the corresponding fields by $\chi^{I}$ again, and their ( $k$ independent) action by $S_{\text {aux }}[\chi ; g]$, we have then

$$
\mathrm{e}^{-\Gamma_{k}^{\text {grav }, 2 \mathrm{D}, \mathrm{NGFP}}[g]} \equiv \int \mathcal{D} \chi \mathrm{e}^{-S_{\text {aux }}[\chi ; g]} \cdot \mathrm{e}^{-N[g]} .
$$

Here $N[g] \propto \int \mathrm{d}^{2} x \sqrt{g}$ is a inessential correction term to make sure that also the nonuniversal cosmological constant terms agree on both sides of (4.17); it depends on the precise definition of the functional integral.

Clearly the auxiliary matter CFT can be chosen in many different ways, the only constraint is that it must have the correct central charge, $c_{\mathrm{aux}}=c_{\mathrm{grav}}^{\mathrm{NGFP}}$, that is, $c_{\mathrm{aux}}=$ $25-N$ or $c_{\text {aux }}=19-N$, respectively. Here are two examples of auxiliary CFTs:

(5a) Minimally coupled scalars. For $c_{\text {aux }}>0$ the simplest choice is a multiplet of minimally coupled scalars $\chi^{I}(x), I=1, \cdots, c_{\text {aux }}$. These auxiliary fields may not be confused with the physical matter fields $A^{i}(x), i=1, \cdots, N$. The $\chi^{\prime}$ 's and $A$ 's have nothing to do with each other except that their respective numbers must add up to 25 (or to 19).

(5b) Feigin-Fuks theory. The induced gravity action $I[g]$ being a non-local functional, it is natural to introduce one, or several fields in addition to the metric that render the action local. The minimal way to achieve this is by means of a single scalar field, $B(x)$, as in Feigin-Fuks theory [68-70], which has a nonminimal coupling to the metric. Consider the following local action, invariant under general coordinate transformations applied to $g_{\mu \nu}$ and $B$ :

$$
I_{\mathrm{loc}}[g, B] \equiv \int \mathrm{d}^{2} x \sqrt{g}\left(D_{\mu} B D^{\mu} B+2 R B\right) .
$$

The equation of motion $\delta I_{\text {loc }} / \delta B=-2 \sqrt{g}(\square B-R)=0$ is solved by $B=B(g) \equiv \square^{-1} R$ which when substituted into $I_{\text {loc }}$ reproduces precisely the non-local form of the induced gravity action: $I_{\mathrm{loc}}[g, B(g)]=\int \sqrt{g} R \square^{-1} R \equiv I[g]$.

As $I_{\text {loc }}$ is quadratic in $B$, the same trick works also quantum mechanically when we perform the Gaussian integration over $B$ rather than solve its field equation. Hence, the exponentiated $\Gamma_{k}^{\text {grav }, 2 \mathrm{D}, \mathrm{NGFP}}$ has the representation

$$
\mathrm{e}^{-\frac{(25-N)}{96 \pi} I[g]+\cdots}=\int \mathcal{D} B \mathrm{e}^{-\frac{(24-N)}{96 \pi} \int \mathrm{d}^{2} x \sqrt{g}\left(D_{\mu} B D^{\mu} B+2 R B+\cdots\right)}
$$


Here again the dots stand for a cosmological constant which depends on the precise definition of the functional measure $\mathcal{D} B$. It is well known that thanks to the $R B$-term the CFT of the $B$-field (in the limit $g_{\mu \nu} \rightarrow \delta_{\mu \nu}$ ) has a shifted central charge [71, 72]; in the present case this reproduces the values (4.15).

So the conclusion is that while the fixed point action is a non-local functional $\propto$ $\int \sqrt{g} R \square^{-1} R$ in terms of the metric alone, one may introduce additional fields such that the same physics is described by a local (concretely, second-derivative) action. In particular, $\Gamma_{k}^{\text {grav,2D,NGFP }}$ and the local functional

$$
\Gamma_{k}^{\mathrm{loc}}[g, B] \equiv \frac{(24-N)}{96 \pi} \int \mathrm{d}^{2} x \sqrt{g}\left(D_{\mu} B D^{\mu} B+2 R B+\cdots\right)
$$

are fully equivalent, even quantum mechanically.

(6) Positivity in the gravitational sector. Pure quantum gravity $(N=0)$ and quantum gravity coupled to less than 25 (or 19) scalars are governed by a fixed point CFT with a positive central charge.

Clearly, this is good news concerning the pressing issue of unitarity (Hilbert space positivity) in asymptotically safe gravity. The theories with with $c_{\text {grav }}^{\mathrm{NGFP}}>0$, continued to Lorentzian signature, do indeed admit a quantum mechanical interpretation and have a state space which is a Hilbert space in the mathematical sense (no negative norm states), supporting a unitary representation of the Virasoro algebra [73].

(6a) Schwinger term. Leaving the analytic continuation to the Lorentzian world aside, it is interesting to note that already in Euclidean space the simple-looking induced gravity action "knows" about the fact that $c_{\mathrm{grav}}^{\mathrm{NGP}}<0$ would create a problem for the probability interpretation. By taking two functional derivatives of the standard effective action (4.6) we can compute the 2-point function $\left\langle T_{\mu \nu}^{\text {grav }}(x) T_{\rho \sigma}^{\text {grav }}(y)\right\rangle$ and, in particular, its contracted form $\left\langle\Theta_{0}(x) \Theta_{0}(y)\right\rangle$. Setting thereafter $g_{\mu \nu}=\delta_{\mu \nu}$, which as we saw is a self-consistent background, and choosing a suitable coordinate chart, we obtain the following Schwinger term:

$$
\left\langle\Theta_{0}(x) \Theta_{0}(y)\right\rangle=-\frac{c_{\mathrm{grav}}^{\mathrm{NGFP}}}{12 \pi} \partial^{\mu} \partial_{\mu} \delta(x-y) .
$$

Let us smear $\Theta_{0}$ with a real valued test function $f$ that vanishes at the boundary and outside of the chart region, or, in the case where the chart is the entire Euclidean plane, falls off rapidly at infinity: ${ }^{10} \Theta_{0}[f] \equiv \int \mathrm{d}^{2} x f(x) \Theta_{0}(x)$. Then, applying $\int \mathrm{d}^{2} x \mathrm{~d}^{2} y f(x) f(y) \cdots$ to both sides of (4.21), we find after an integration by parts:

$$
\left\langle\Theta_{0}[f]^{2}\right\rangle=+c_{\text {grav }}^{\mathrm{NGFP}} \frac{1}{12 \pi} \int \mathrm{d}^{2} x\left(\partial_{\mu} f\right) \delta^{\mu \nu}\left(\partial_{\nu} f\right) .
$$

Since the integral on the r.h.s. of (4.22) is manifestly positive, we conclude that if $c_{\mathrm{grav}}^{\mathrm{NGF}}<0$ the expectation value of the square $\Theta_{0}[f]^{2}$ is negative. Clearly this would be problematic already in the context of statistical mechanics (at least with real field variables).

\footnotetext{
${ }^{10}$ Note that in the latter case the function $f$ has support on the entire Euclidean plane, hence we are clearly not testing Osterwalder-Schrader [4, 5] reflection positivity here [6, 7].
} 
(6b) Induced gravity approach in 4D: a comparison. Note that one can extract the central charge from the Schwinger term by performing an integral $\int \mathrm{d}^{2} x x^{2}(\cdots)$ over both sides of eq. (4.21). Since Newton's constant is dimensionless in $2 \mathrm{D}$, and $\breve{G}^{-1}=\breve{g}_{*}^{-1}=$ $b=\frac{2}{3} c_{\mathrm{grav}}^{\mathrm{NGP}}$, this leads to the following integral representation for the Newton constant belonging to the $2 \mathrm{D}$ world governed by the FP trajectory [74]:

$$
\breve{G}^{-1}=-2 \pi \int \mathrm{d}^{2} x x^{2}\left\langle\Theta_{0}(x) \Theta_{0}(0)\right\rangle .
$$

It is interesting to note that this representation is of precisely the same form as the relations that had been derived long ago within the induced gravity approach in $4 \mathrm{D}$, the hope being that ultimately one should be able to compute its r.h.s. from a matter field theory, assumed known, the Standard Model, say, and would then predict the value of Newton's constant in terms of matter-related constants of Nature.

For a review and a discussion of the inherent difficulties we refer to [74]. We see that, in a sense, Asymptotic Safety was successful in making this scenario work, producing a positive Newton constant in particular, but with one key difference: the underlying matter field theory, here the 'aux' system, is no longer an arbitrary external input, but is chosen so as to reproduce the NGFP action, an object computed from first principles.

(7) Complete vs. gauge invariant fixed point functional. So far we mainly focused on the gravitational part of the NGFP functional. The complete EAA, namely $\Gamma_{k}=$ $\Gamma_{k}^{\text {grav }}+\Gamma_{k}^{\mathrm{M}}+\Gamma_{k}^{\mathrm{gf}}+\Gamma_{k}^{\mathrm{gh}}$ contains matter, gauge fixing and ghost terms in addition. But since the present truncation neglects the running of the latter three parts, they may be considered always at their respective fixed point. Also, they have an obvious interpretation in $2 \mathrm{D}$ exactly. Furthermore, our truncation assumes that neither $\Gamma_{k}^{\text {grav }}$ nor $\Gamma_{k}^{\mathrm{M}}$ as given in (1.3) has an "extra" $\bar{g}$-dependence.

As a result, the sum of gravity and matter ('GM') contributions,

$$
\Gamma_{k}^{\mathrm{GM}, 2 \mathrm{D}}[g, A] \equiv \Gamma_{k}^{\text {grav }, 2 \mathrm{D}}[g]+\frac{1}{2} \sum_{i=1}^{N} \int \mathrm{d}^{2} x \sqrt{g} g^{\mu \nu} \partial_{\mu} A^{i} \partial_{\nu} A^{i},
$$

enjoys both Background Independence, here meaning literally independence of the background metric, and Gauge Invariance, i.e. it does not change under diffeomorphisms applied to $g_{\mu \nu}$ and $A^{i}$.

Thanks to the second property, ${ }^{11}$ we may adopt the point of view that it is actually the gauge invariant functional $\Gamma_{k}^{\mathrm{GM}, 2 \mathrm{D}}$ alone which contains all information of interest and was thus "handed over" alone from the higher dimensional Einstein-Hilbert world to the intrinsically 2-dimensional induced gravity setting. Therefore, if in 2D the necessity of gauge fixing arises, we can in principle pick a new gauge, different from the one employed in $d>2$ for the computation of the $\beta$-functions. ${ }^{12}$

\footnotetext{
${ }^{11}$ Which might not be realized in more complicated truncations!

${ }^{12}$ This could not be done if one wants to combine loop or RG calculations from $d>2$ with others done in $d=2$ exactly. However, in the present paper all dynamical calculations are done in $d>2$, i.e. before the 2D limit is taken.
} 
(8) Unitarity vs. stability: the conformal factor "problem". Next we take advantage of the particularly convenient conformal gauge available in strictly 2 dimensions (cf. section 3.1), and evaluate $\Gamma_{k}^{\text {grav }, 2 \mathrm{D}, \mathrm{NGFP}}[g]$ as given explicitly by eq. (4.5) for metrics of the special form $g_{\mu \nu}=\hat{g}_{\mu \nu} \mathrm{e}^{2 \phi}$. The result is a Liouville action as before in eqs. (2.22), (2.21), this time without any undetermined piece such as $U_{k}[\hat{g}]$, however:

$$
\begin{aligned}
\Gamma_{k}^{\text {grav }, 2 \mathrm{D}, \mathrm{NGFP}}\left[\hat{g} \mathrm{e}^{2 \phi}\right] & \equiv \frac{c_{\text {grav }}^{\mathrm{NGFP}}}{96 \pi} I[\hat{g}]+\Gamma_{k}^{\mathrm{L}}[\phi ; \hat{g}] \\
\Gamma_{k}^{\mathrm{L}}[\phi ; \hat{g}] & =\frac{c_{\mathrm{grav}}^{\mathrm{NGFP}}}{12 \pi} \int \mathrm{d}^{2} x \sqrt{\hat{g}}\left\{-\frac{1}{2} \hat{D}_{\mu} \phi \hat{D}^{\mu} \phi-\frac{1}{2} \hat{R} \phi+\breve{\lambda}_{*} k^{2} \mathrm{e}^{2 \phi}\right\} .
\end{aligned}
$$

Since $c_{\text {grav }}^{\mathrm{NGFP}}=25-N$ (or $c_{\text {grav }}^{\mathrm{NGFP}}=19-N$ with the linear parametrization), we observe that for pure gravity, and gravity interacting with not too many matter fields, the conformal factor has a "wrong sign" kinetic term that might seem to indicate an instability at first sight. If we think of the fixed point action as induced by some auxiliary CFT with central charge $c_{\text {aux }}=c_{\text {grav }}^{\mathrm{NGFP}}=25-N>0$, we see that this is exactly the correlation mentioned in paragraph $(\mathbf{3 b})$ above: bona fide unitary CFTs generate "wrong sign" kinetic terms for the conformal factor.

We emphasize that the unstable $\phi$-action is neither unexpected, nor "wrong" from the physics point of view, nor in contradiction with the positive central charge of the fixed point CFT. Let us discuss these issues in turn now.

(8a) The importance of Gauss' law. Recall the standard count of gravitational degrees of freedom in Einstein-Hilbert gravity: in $d$ dimensions, the symmetric tensor $g_{\mu \nu}$ contains $\frac{1}{2} d(d+1)$ unknown functions which we try to determine from the $\frac{1}{2} d(d+1)$ field equations $G_{\mu \nu}=\cdots$. Those are not independent, but subject to $d$ Bianchi identities. Moreover, we need to impose $d$ coordinate conditions due to diffeomorphism invariance. This leaves us with $N_{\mathrm{EH}}(d) \equiv \frac{1}{2} d(d+1)-d-d=\frac{1}{2} d(d-3)$ gravitational degrees of freedom, meaning that by solving the Cauchy problem for $g_{\mu \nu}$ we can predict the time evolution of $N_{\mathrm{EH}}(d)$ functions that, (i), are related to "physical " (i.e. gauge invariant) properties of space, (ii), are algebraically independent among themselves, and (iii), are independent of the functions describing the evolution of matter.

With $N_{\mathrm{EH}}(4)=2$ we thus recover the gravitational waves of $4 \mathrm{D}$ General Relativity, having precisely 2 polarization states. Similarly, $N_{\mathrm{EH}}(3)=0$ tells us that there can be no gravitational waves in 3 dimensions since all independent, gauge invariant properties described by the metric can be inferred already from the matter evolution. No extra initial conditions can, or must, be imposed.

Finally $N_{\mathrm{EH}}(2)=-1$ seems to suggest that "gravity has -1 degree of freedom in 2 dimensions". Strange as it might sound, the meaning of this result is quite clear: the quantum metric with its ghosts removes one degree of freedom from the matter system. If, in absence of gravity, the Cauchy problem of the matter system has a unique solution after specifying $N_{\mathrm{M}}$ initial conditions, then this number gets reduced to $N_{\mathrm{M}}-1$ by coupling the system to gravity.

Quantum mechanically, on a state space with an indefinite metric, the removing of degrees of freedom happens upon imposing "Gauss law constraints", or "physical state 
conditions" on the states. As a result, the potentially dangerous negative-norm states due to the wrong sign of the kinetic term of $\phi$ are not part of the actual (physical) Hilbert space. The latter can be built using matter operators alone, and it is smaller in fact than without gravity. ${ }^{13}$

The situation is analogous to Quantum Electrodynamics (QED) in Coulomb gauge, for example. The overall sign of the Maxwell action $\propto F_{\mu \nu} F^{\mu \nu}$ is chosen such that the spatial components of $A_{\mu}$ have a positive kinetic term, and so it is unavoidable that the time component $A_{0}$ has a negative one, like the conformal factor in (4.26). However, it is well known [76] that the states with negative (norm) ${ }^{2}$ generated by $A_{0}$ do not survive imposing Gauss' law $\boldsymbol{\nabla} \cdot \boldsymbol{E}=e \psi^{\dagger} \psi$ on the states. This step indeed removes one degree of freedom since $A_{0}$ and $\rho_{\mathrm{em}} \equiv e \psi^{\dagger} \psi$ get coupled by an instantaneous equation, $\nabla^{2} A_{0}(t, \boldsymbol{x})=$ $-\rho_{\mathrm{em}}(t, \boldsymbol{x})$.

(8b) Instability and attractivity of classical gravity. To avoid any misunderstanding we recall that in constructing realistic $4 \mathrm{D}$ theories of gravity it would be quite absurd, at least in the Newtonian limit, to "solve" the problem of the conformal factor by manufacturing a positive kinetic term for it in some way. In taking the classical limit of General Relativity this kinetic term descends essentially to the $\nabla \varphi_{\mathrm{N}} \cdot \boldsymbol{\nabla} \varphi_{\mathrm{N}}$-part of the classical Lagrangian governing Newton's potential $\varphi_{\mathrm{N}}$ and therefore fixes the positive sign on the r.h.s. of Poisson's equation, $\nabla^{2} \varphi_{\mathrm{N}}=+4 \pi G \rho$. However, this latter plus sign expresses nothing less than the universal attractivity of classical gravity, something we certainly want to keep.

This simple example shows that the conformal factor instability is by no means an unmistakable sign for a physical deficiency of the theory under consideration. The theory can be perfectly unitary if there are appropriate Gauss' law-type constraints to cut out the negative norm states of the indefinite metric state space.

(8c) Central charge in Liouville theory. Finally, we must discuss a potential source of confusion concerning the correct identification of the fixed point's central charge. Let us pretend that the Liouville action $\Gamma_{k}^{\mathrm{L}}[\phi ; \hat{g}]$ describes a matter field $\phi$ in a "background" metric $^{14} \hat{g}_{\mu \nu}$. It would then be natural to ascribe to this field the stress tensor

$$
T_{k}^{\mathrm{L}}[\phi ; \hat{g}]^{\mu \nu} \equiv \frac{2}{\sqrt{\hat{g}}} \frac{\delta \Gamma_{k}^{\mathrm{L}}[\phi ; \hat{g}]}{\delta \hat{g}_{\mu \nu}}
$$

Without using the equation of motion (i.e. "off shell") its trace is given by

$$
\Theta_{k}^{\mathrm{L}}[\phi ; \hat{g}] \equiv \hat{g}_{\mu \nu} T_{k}^{\mathrm{L}}[\phi ; \hat{g}]^{\mu \nu}=\frac{c_{\mathrm{grav}}^{\mathrm{NGFP}}}{12 \pi}\left(\hat{\square} \phi+2 \breve{\lambda}_{*} k^{2} \mathrm{e}^{2 \phi}\right)
$$

Concerning (4.28), several points are to be noted.

\footnotetext{
${ }^{13}$ See Polchinski [75] for a related discussion.

${ }^{14}$ Recall, however, that the reference metric $\hat{g}_{\mu \nu}$ that enters only the conformal parametrization of $2 \mathrm{D}$ metrics is to be distinguished carefully from the true background metric $\bar{g}_{\mu \nu}$ which is at the heart of the entire gravitational EAA setting. In this conformal parametrization, a generic bi-metric action $F[g, \bar{g}]$ translates into a functional of two conformal factors, $F[\phi, \bar{\phi} ; \hat{g}] \equiv F\left[\hat{g} \mathrm{e}^{2 \phi}, \hat{g} \mathrm{e}^{2 \bar{\phi}}\right]$.
} 
(i). Varying $\Gamma_{k}^{\mathrm{L}}$ with respect to $\phi$ yields Liouville's equation $\hat{\square} \phi+2 \breve{\lambda}_{*} k^{2} \mathrm{e}^{2 \phi}=\frac{1}{2} \hat{R}$. With $\phi_{\text {sol }}$ denoting any solution to it we obtain "on shell" the following $k$-independent trace:

$$
\Theta^{\mathrm{L}}\left[\phi_{\text {sol }} ; \hat{g}\right]=+c_{\text {grav }}^{\mathrm{NGFP}} \frac{1}{24 \pi} \hat{R} .
$$

If we now compare (4.29) to the general rule (4.10) we conclude that the Liouville field represents a CFT with the central charge

$$
c^{\mathrm{L}}=-c_{\text {grav }}^{\mathrm{NGFP}}
$$

which is negative for pure asymptotically safe gravity, namely $c^{\mathrm{L}}=-25$, or -19 , respectively.

Does this result indicate that the fixed point CFT is non-unitary, after all? The answer is a clear 'no', and the reason is as follows.

(ii). The Liouville theory governed by $\Gamma_{k}^{\mathrm{L}}$ of (4.26) is not a faithful description of the NGFP. According to eq. (4.25), the full action contains the "pure gravity" term $\frac{c_{\mathrm{grav}}^{\mathrm{NGF}}}{96 \pi} I[\hat{g}]$ in addition. In order to correctly identify the central charge of the NGFP it is essential to add the $\hat{g}_{\mu \nu}$-derivative of this term to the Liouville stress tensor. Hence, the trace (4.28) gets augmented to

$$
\begin{aligned}
\frac{2 \hat{g}_{\mu \nu}}{\sqrt{\hat{g}}} \frac{\delta}{\delta \hat{g}_{\mu \nu}}\left(\frac{c_{\mathrm{grav}}^{\mathrm{NGF}}}{96 \pi} I[\hat{g}]\right)+\Theta_{k}^{\mathrm{L}}[\phi ; \hat{g}] & =-\frac{c_{\mathrm{grav}}^{\mathrm{NGFP}}}{24 \pi} R(\hat{g})+\Theta_{k}^{\mathrm{L}}[\phi ; \hat{g}] \\
& =\frac{c_{\mathrm{grav}}^{\mathrm{NGFP}}}{24 \pi}\left[-R(\hat{g})+2 \hat{\square} \phi+4 \breve{\lambda}_{*} k^{2} \mathrm{e}^{2 \phi}\right] \\
& =\frac{c_{\mathrm{grav}}^{\mathrm{NGF}}}{24 \pi}\left[-\mathrm{e}^{-2 \phi}(R(\hat{g})-2 \hat{\square} \phi)+4 \breve{\lambda}_{*} k^{2}\right] \mathrm{e}^{2 \phi} \\
& =\frac{c_{\mathrm{grav}}^{\mathrm{NGFP}}}{24 \pi}\left[-R\left(\hat{g} \mathrm{e}^{2 \phi}\right)+4 \breve{\lambda}_{*} k^{2}\right] \mathrm{e}^{2 \phi} \\
& =\mathrm{e}^{2 \phi} \Theta_{k}\left[\hat{g} \mathrm{e}^{2 \phi}\right] .
\end{aligned}
$$

In the $2^{\text {nd }}$ line of $(4.31)$ we inserted (4.28), in going from the $3^{\text {rd }}$ to the $4^{\text {th }}$ line we exploited the identity (A.11) from the appendix, and in the last line we used (4.16). So with this little calculation we have checked that the Liouville stress tensor makes physical sense only when combined with the pure gravity piece. ${ }^{15}$ If this is done, the total gravitational trace from which the correct central charge is inferred, eq. (4.16), is indeed recovered, as it should be. It satisfies the relation ${ }^{16}$

$$
\Theta_{k}[g] \equiv \Theta_{k}\left[\hat{g} \mathrm{e}^{2 \phi}\right]=\mathrm{e}^{-2 \phi}\left(-\frac{c_{\mathrm{grav}}^{\mathrm{NGFP}}}{24 \pi} \hat{R}+\Theta_{k}^{\mathrm{L}}[\phi ; \hat{g}]\right),
$$

which holds true even off shell.

\footnotetext{
${ }^{15}$ In isolation, $\Theta^{\mathrm{L}}[\phi ; \hat{g}]$ is not invariant under the split-symmetry transformations (3.3), i.e. not a function of the combination $\hat{g} \mathrm{e}^{2 \phi}$ only.

${ }^{16}$ The explicit factor $\mathrm{e}^{-2 \phi}$ in (4.32) is simply due to the different volume elements $\sqrt{\hat{g}}$ and $\sqrt{g}=\sqrt{\hat{g}} \mathrm{e}^{2 \phi}$ appearing in the definitions of the stress tensors (4.27) and (4.9), respectively.
} 
(iii). If we take $\phi$ on shell, eq. (4.29) applies and so the two terms in the brackets of eq. (4.32) cancel precisely. This, too, is as it should be since from eq. (4.8) we know already that $\Theta_{k}[g]$ vanishes identically when $g \equiv \bar{g}$ is a self-consistent background, and this is exactly what we insert into (4.32) when $\phi$ is a solution of Liouville's equation.

Thus, taking the above points together we now understand that nothing is wrong with $c^{\mathrm{L}}=-c_{\text {grav }}^{\mathrm{NGFP}}$. In fact, $c^{\mathrm{L}}<0$ for pure gravity is again a reflection of the Liouville field's "wrong-sign" kinetic term ${ }^{17}$ and its perfectly correct property of reducing the total number of degrees of freedom.

\section{Status of different field parametrizations}

To fully establish the properties of the NGFP in exactly 2 dimensions, it remains to understand the status and reliability of the exponential and linear field parametrizations, respectively. Why is it the former that reproduces the results of standard conformal field theory, including the bosonic critical dimension, while the latter fails to do so $[22,26,57,58]$ ?

\subsection{Different universality classes?}

It might be appropriate to begin with a word of warning: for the time being, it is not clear whether the exponential and the linear parametrization, respectively, describe the same physics at the exact level. We cannot fully exclude the possibility that both of them are equally correct, but probe instead two different universality classes.

It is a notoriously difficult question in virtually all functional integral ${ }^{18}$ based approaches to quantum gravity whether, or to what extent, degenerate, wrong-signature or even vanishing tensor fields should be included [78]. Since the set of nondegenerate metrics with fixed signature forms a nonempty open subset in the space of all covariant symmetric 2-tensor fields [59], there is no a priori reason to expect that it has vanishing measure, and so this question has no obvious answer. It is known, however, that "sufficiently different" choices will lead to inequivalent theories [79].

In this context it might well be that a NGFP in a theory which is based only on nondegenerate fixed-signature metrics falls into another universality class than a fixed point in a theory which includes all covariant symmetric tensors without restrictions concerning degeneracy and signature. If so, the properties of the respective NGFP, in particular the values of the central charge, could be different then.

Interestingly enough, the two parametrizations considered here give rise to these two different options, namely genuine metrics vs. unconstrained symmetric tensors:

\footnotetext{
${ }^{17}$ Hence, at the technical level, the wrong-sign kinetic term requires special attention (regularization, analytic continuation, or similar) at intermediate steps of the calculation at most.

${ }^{18}$ It is well known that standard 1D configuration space functional integrals are dominated by nondifferentiable paths since the set of differentiable ones has measure 0. The basic laws of quantum mechanics, noncommutativity of positions and momenta, force us to include these classically forbidden nondifferentiable trajectories in the path integral [77]. Similarly, a consistent gravitational path integral might require integrating over "metrics" which have further nonclassical features to a degree that is to be found out.
} 
(a). In ref. [59] it was argued that the exponential parametrization is adapted to the nonlinear structure of the field space $\mathcal{F}$ of all pure metrics, i.e. objects in $\mathcal{F}$ are nondegenerate and have a prescribed signature. The nondegeneracy and signature requirements prevent $\mathcal{F}$ from exhibiting vector space character. As a consequence, it is not possible to simply add an arbitrary fluctuation to a given metric in order to obtain another metric since it may happen that one leaves the set $\mathcal{F}$ owing to the addition. To reach another metric one rather has to start from a given metric and move along geodesics. Only then it is ensured that all metrics parametrized this way satisfy the signature and nondegeneracy constraint. It can be shown that there is a canonical connection on $\mathcal{F}$ adjusted to this constraint, and that the resulting geodesics are parametrized precisely by the exponential relation $g_{\mu \nu}=\bar{g}_{\mu \rho}\left(\mathrm{e}^{h}\right)^{\rho}{ }_{\nu}$ with a symmetric tensor field $h_{\mu \nu}$ [59]. This explains the special status of the exponential parametrization.

(b). The linear parametrization, $g_{\mu \nu}=\bar{g}_{\mu \nu}+h_{\mu \nu}$, on the other hand, allows integrating over a much larger space of field configurations including degenerate, wrong-signature and vanishing tensor fields: ${ }^{19}$ Since $h_{\mu \nu}$ can be any symmetric tensor field, it is clear that the set of pure metrics can be left by adding it to $\bar{g}_{\mu \nu}$.

Thus, the two parametrizations fall indeed into qualitatively different categories, and there is at least the possibility that future RG studies might show that the respective NGFPs refer to different universality classes. We conjecture that these classes would then be represented by $c_{\mathrm{grav}}^{\mathrm{NGFP}}=19$ for the linear parametrization and by $c_{\mathrm{grav}}^{\mathrm{NGP}}=25$ if the metric is parametrized by an exponential. In any case, the issue of parametrization dependence [80] should always be reconsidered when a better truncation becomes technically manageable. ${ }^{20}$

We believe that although at this stage no parametrization of the metric should be preferred over the other one, the compatibility of EAA-based Asymptotic Safety results with other approaches to quantum gravity can in principle discriminate between different parametrizations. The critical value ' 25 ' for the central charge indicates indeed that our calculations based on the exponential parametrization are closer to those of conformal field theory. In the next subsection we aim at presenting another argument suggesting that the exponential parametrization is particularly appropriate in the $2 \mathrm{D}$ limit.

\subsection{The birth of exponentials in $2 \mathrm{D}$}

The argument in this subsection considers only such dynamical metrics $g_{\mu \nu}$ that are conformally related to a fixed reference metric $\hat{g}_{\mu \nu}$, and only their relative conformal factor is quantized. The resulting "conformally reduced" setting [83, 84] amounts to the exact theory in 2D, but it is an approximation in higher dimensions. (Accordingly, "exponential parametrization" refers to the form of the conformal factor in the following.)

\footnotetext{
${ }^{19}$ This would be in the spirit of ref. [78], and one might expect to find a phase of unbroken diffeomorphism invariance, among others.

${ }^{20} \mathrm{~A}$ first indication pointing towards the possibility of different classes might be contained in recent results from the $f(R)$-truncation in $4 \mathrm{D}$ where an apparently parametrization dependent number of relevant directions was observed [81, 82].
} 
Among all possible ways of parametrizing the conformal factor there exists a distinguished parametrization in each dimension $d$.

(1) Distinguished parametrizations. Let us consider the conformal reduction of the Einstein-Hilbert action $S_{\mathrm{EH}}[g] \equiv-\frac{1}{16 \pi G} \int \mathrm{d}^{d} x \sqrt{g}(R-2 \Lambda)$ in any number of dimensions $d>2$. That is, we evaluate $S_{\mathrm{EH}}$ only on metrics which are conformal to a given $\hat{g}$ consistent with the desired topology. But how should we write the factor relating $g$ and $\hat{g}$ now? Assume, for instance, the reduced $S_{\mathrm{EH}}$ plays the role of a bare action under a functional integral over a certain field $\Omega$ representing the conformal factor, how then should the latter be written in terms of $\Omega$ ? Clearly, infinitely many parametrizations are possible here, and depending on our choice the reduced $S_{\mathrm{EH}}$ will look differently.

There exists a distinguished parametrization, however, which is specific to the dimensionality $d$, having the property that $\int \sqrt{g} R$ becomes quadratic in $\Omega$. Starting out from a power ansatz, $g_{\mu \nu}=\Omega^{2 \nu} \hat{g}_{\mu \nu}$, the integral $\int \sqrt{g} R$ will in general produce a potential term $\propto \hat{R}$ times a particular power of $\Omega$, and a kinetic term $\propto(\hat{D} \Omega)^{2}$ times another power of $\Omega$. The exponent of the latter turns out to be zero, yielding a kinetic term quadratic in $\Omega$, precisely if [85]

$$
\nu=2 /(d-2), \quad g_{\mu \nu}=\Omega^{4 /(d-2)} \hat{g}_{\mu \nu} .
$$

In this case the potential term is found to be quadratic as well, and one obtains $[83,85]$

$$
S_{\mathrm{EH}}\left[g=\Omega^{4 /(d-2)} \hat{g}\right]=-\frac{1}{8 \pi G} \int \mathrm{d}^{d} x \sqrt{\hat{g}}\left[\frac{1}{2 \xi(d)} \hat{D}_{\mu} \Omega \hat{D}^{\mu} \Omega+\frac{1}{2} \hat{R} \Omega^{2}-\Lambda \Omega^{2 d /(d-2)}\right] .
$$

Here we introduced the constant

$$
\xi(d) \equiv \frac{(d-2)}{4(d-1)}
$$

Usually one employs $\Omega(x)-1 \equiv \omega(x)$ rather than $\Omega$ itself as the dynamical field that is quantized, i.e. integrated over if $S_{\mathrm{EH}}$ appears in a functional integral. Then there will be no positivity issues as long as $\omega(x)$ stays small. We emphasize, however, that the derivation of neither (5.2) nor the related action for $\omega$,

$$
S_{\mathrm{EH}}[\omega ; \hat{g}]=-\frac{1}{8 \pi G} \int \mathrm{d}^{d} x \sqrt{\hat{g}}\left[\frac{1}{2 \xi(d)} \hat{g}^{\mu \nu} \partial_{\mu} \omega \partial_{\nu} \omega+\frac{1}{2} \hat{R}(1+\omega)^{2}-\Lambda(1+\omega)^{2 d /(d-2)}\right],
$$

involves any (small field, or other) expansion. (It involves an integration by parts though, hence there could be additional surface contributions if spacetime has a boundary.)

(2) Metric operators. The exponent appearing in the conformal factor $\Omega^{2 \nu}$ is noninteger in general, exceptions being $d=3,4$, and 6 , see table 1 .

The virtue of a quadratic action needs no mentioning, of course. As long as the cosmological constant plays no role $-\Lambda$ will always give rise to an interaction term - the computation of the RG flow will be easiest and most reliable if we employ the distinguished parametrization. ${ }^{21}$

\footnotetext{
${ }^{21}$ The RG flow of the conformally reduced Einstein-Hilbert truncation ("CREH") with the distinguished parametrizations has been computed in [83], an LPA-type extension was considered in [84], see also [86].
} 


\begin{tabular}{|cccc|}
\hline$d$ & 3 & 4 & 6 \\
\hline Conformal factor & $\Omega^{4}$ & $\Omega^{2}$ & $\Omega$ \\
Volume operator & $\Omega^{6}$ & $\Omega^{4}$ & $\Omega^{3}$ \\
\hline
\end{tabular}

Table 1. Conformal factor and volume operator for the distinguished parametrization.

One should be aware that there is a conservation of difficulties also here. Generically the conformal factor depends on the quantum field nonlinearly. Hence, canonically speaking, even if the action is trivial (Gaussian), the construction of a metric operator amounts to defining $\Omega^{2 \nu}$ or $(1+\omega)^{2 \nu}$ as a composite operator. And in fact, the experience with models such as Liouville theory [87-89] shows how extremely difficult this can be.

At present we are just interested in comparing the relative degree of reliability of two truncated RG flows, based upon different field parametrizations. For this purpose it is sufficient to learn from the above argument that the "most correct" results should be those from the distinguished parametrization (5.1) since then the theory is free (for $\Lambda=0$ ). But what is the distinguished parametrization in 2 dimensions?

(3) The limit $\boldsymbol{d} \rightarrow \mathbf{2}$. As we lower $d$ towards two dimensions, the distinguished form of the conformal factor, $(1+\omega)^{4 /(d-2)}$, develops into a function which increases with $\omega$ faster than any power. At the same time the constant $\xi(d)$ goes to zero, and (5.4) becomes

$$
\begin{array}{r}
S_{\mathrm{EH}}[\omega ; \hat{g}]=-\frac{1}{16 \pi \breve{G}} \int \mathrm{d}^{2+\varepsilon} x \sqrt{\hat{g}}\left[\frac{4}{\varepsilon^{2}} \hat{g}^{\mu \nu} \partial_{\mu} \omega \partial_{\nu} \omega\{1+\mathcal{O}(\varepsilon)\}\right. \\
+\frac{1}{\varepsilon} \hat{R}(1+\omega)^{2}-2 \breve{\Lambda}(1+\omega)^{2(2+\varepsilon) / \varepsilon] .}
\end{array}
$$

Here we wrote $G \equiv \breve{G} \varepsilon$ and $\Lambda \equiv \breve{\Lambda} \varepsilon$ again, assuming that $\breve{G}, \breve{\Lambda}=\mathcal{O}\left(\varepsilon^{0}\right)$. We see that in order to obtain a meaningful kinetic term we must rescale $\omega$ by a factor of $\varepsilon$ prior to taking the limit $\varepsilon \searrow 0$.

Introducing the new field $\phi(x) \equiv 2 \omega(x) / \varepsilon$, its kinetic term $\hat{g}^{\mu \nu} \partial_{\mu} \phi \partial_{\nu} \phi\{1+\mathcal{O}(\varepsilon)\}$ will have a finite and nontrivial limit. The concomitant conformal factor $\Omega^{2 \nu}$ has the limit

$$
\lim _{\varepsilon \rightarrow 0}(1+\omega)^{4 / \varepsilon}=\lim _{\varepsilon \rightarrow 0}\left(1+\frac{1}{2} \varepsilon \phi\right)^{4 / \varepsilon}=\lim _{n \rightarrow \infty}\left(1+\frac{2 \phi}{n}\right)^{n}=\mathrm{e}^{2 \phi} .
$$

This demonstrates that the exponential parametrization $g_{\mu \nu}=\mathrm{e}^{2 \phi} \hat{g}_{\mu \nu}$ is precisely the $2 D$ limit of the distinguished (power-like) parametrizations in $d>2$.

The cosmological term in (5.5) involves the same exponential for $d \rightarrow 2$, and the originally quadratic potential $\hat{R}(1+\omega)^{2}$ turns into a linear one for $\phi$. Taking everything together the Laurent series of $S_{\mathrm{EH}}$ in $\varepsilon$ looks as follows:

$$
S_{\mathrm{EH}}[\phi ; \hat{g}]=-\frac{1}{16 \pi \breve{G}}\left\{\frac{1}{\varepsilon} \int \mathrm{d}^{2+\varepsilon} x \sqrt{\hat{g}} \hat{R}+\int \mathrm{d}^{2} x \sqrt{\hat{g}}\left(\hat{g}^{\mu \nu} \partial_{\mu} \phi \partial_{\nu} \phi+\hat{R} \phi-2 \breve{\Lambda} \mathrm{e}^{2 \phi}\right)\right\}+\mathcal{O}(\varepsilon) .
$$

The first term on the r.h.s. is $\phi$-independent and involves a purely topological contribution proportional to the Euler characteristic, which was already encountered in section 3. Ob- 
viously from (5.7) we reobtain Liouville theory as the intrinsically 2D part of the EinsteinHilbert action, but this is perhaps not too much of a surprise.

What is important, though, is that in this derivation, contrary to the discussion in the previous sections, the exponential field dependence of the conformal factor was not put in by hand, we rather derived it.

Here our input were the following two requirements: first, the scaling limit of $S_{\mathrm{EH}}$ should be both non-singular and nontrivial, and second, it should go through a sequence of actions which, apart from the cosmological term, are at most quadratic in the dynamical field. Being quadratic implies that when $S_{\mathrm{EH}}[\omega ; \hat{g}]$ is used as the (conformal reduction of the) Einstein-Hilbert truncation, this truncation is "perfect" at any $\varepsilon$.

Therefore, we believe that using the exponential parametrization already in "higher" dimensions $d>2$ yields more reliable results for the $\beta$-functions and their 2D limits than using the linear parametrization in $d>2$ and taking the $2 \mathrm{D}$ limit of the corresponding $\beta$-functions. (There is still a minor source of uncertainty due to the ghost sector. In either parametrization there are ghost-antighost-graviton interactions which are not treated exactly by the truncations we use.)

The basic difference between the two parametrizations can also be seen quite directly. If we insert $g=\mathrm{e}^{2 \phi} \hat{g}$ into $S_{\mathrm{EH}}$, the resulting derivative term reads exactly, i.e. without any expansion in $\varepsilon$ and/or $\phi$ and rescaling of $\phi$ :

$$
-\frac{(d-1)}{16 \pi \breve{G}} \int \mathrm{d}^{d} x \sqrt{\hat{g}} \mathrm{e}^{(d-2) \phi}(\hat{D} \phi)^{2} .
$$

For $d \rightarrow 2$ this term has a smooth limit (we did use $G=\breve{G} \varepsilon$ after all) and this limit is quadratic in $\phi$.

On the other hand, inserting the linear parametrization $g=(1+\omega) \hat{g}$ into $S_{\mathrm{EH}}$ we obtain again exactly, i.e. without expanding in $\varepsilon$ and/or $\omega$ and rescaling $\omega$ :

$$
-\frac{(d-1)}{64 \pi \breve{G}} \int \mathrm{d}^{d} x \sqrt{\hat{g}}(1+\omega)^{(d-2) / 2} \frac{(\hat{D} \omega)^{2}}{(1+\omega)^{2}} .
$$

The term (5.9), too, has a smooth limit $d \rightarrow 2$, but it is not quadratic in the dynamical field. This renders the $\omega$-theory interacting and makes it a nontrivial challenge for the truncation.

(4) The dimension $\boldsymbol{d}=\mathbf{6}$. As an aside we mention that according to table 1 the case $d=6$ seems to be easiest to deal with since in the preferred field parametrization the conformal factor is linear in the quantum field, and so there is no need to construct a composite operator. The kinetic term (5.9) becomes quadratic exactly at $d=6$.

It is intriguing to speculate that this observation is related to the following rather surprising property enjoyed by the $\beta$-functions derived from the bi-metric Einstein Hilbert truncation (see appendix A.1 of ref. [3]): if $d=6$, and if in addition the dimensionful dynamical cosmological constant $\Lambda^{\mathrm{Dyn}}$ is zero, then the gravity contributions to the $\beta$-functions of both $\Lambda^{\text {Dyn }}$ and the dimensionful dynamical Newton constant $G^{\text {Dyn }}$ vanish exactly. (There are nonzero ghost contributions, though.) 
(5) Summary. On the basis of the above arguments we conclude that most probably the exponential parametrization is more reliable in $2 D$ than the linear one. We believe in particular that $c_{\mathrm{grav}}^{\mathrm{NGFP}}=25$ is more likely to be a correct value of the central charge at the pure gravity fixed point than its competitor '19'. Depending on the reliability of the linear parametrization, the ' 19 ' could be a poor approximation to '25', or a hint at another universality class.

\section{The reconstructed functional integral}

For the following it is important to keep in mind that the "derivation" of the FRGE from a functional integral is only formal as it ignores all difficulties specific to the UV limit of quantum field theories. In fact, rather than the integral, the starting point of the EAA based route to a fundamental theory is the mathematically perfectly well defined, UV cutoff-free flow equation (1.1). In this setting, the problem of the UV limit is shifted from the properties of the equation itself to those of its solutions, converting renormalizability into a condition on the existence of fully extended RG trajectories on theory space. The Asymptotic Safety paradigm is a way of achieving full extendability in the UV and, barring other types of (infrared, etc.) difficulties, it leads to a well-behaved action functional $\Gamma_{k}$ at each $k \in[0, \infty)$.

\subsection{The reconstruction process}

While every complete RG trajectory defines a quantum field theory (with the cutoff(s) removed), it does not give rise to a functional integral description of this theory a priori. Nevertheless, one may ask for a functional integral reproducing a given complete $\Gamma_{k}$-trajectory. This "reconstruction" step has been motivated and discussed in detail in ref. [9].

The association of a functional integral, i.e. a bare theory, to a $\Gamma_{k}$-trajectory is highly non-unique. The first decision to be taken concerns the variables of integration: they may or may not be fields of the same sort as those serving as arguments of $\Gamma_{k}$. From the practical point of view the most important situation is when the integration variables are no (discretized) fields at all, but rather belong to a certain statistical mechanics model whose partition function at criticality is supposed to reproduce the predictions of the EAA trajectory. Besides the nature of the integration variables, a UV regularization scheme, a correspondingly regularized functional integration measure, and an associated bare action $S_{\Lambda}$ are to be chosen. Then the information encapsulated in $\Gamma_{k \rightarrow \infty}$ can be used in order to find out how the bare parameters contained in $S_{\Lambda}$ must depend on the UV cutoff $\Lambda$ in order to give rise to a well-defined path integral reproducing the EAA-trajectory in the limit $\Lambda \rightarrow \infty$.

In [9] this reconstruction has been carried out explicitly for metric gravity in the Einstein-Hilbert truncation for the following choices:

(i). The integration variable is taken to be the metric fluctuation $h_{\mu \nu}$, i.e. (accidentally) the same sort of variable as in the argument of $\Gamma_{k}$. 
(ii). The UV regularization is implemented by means of a sharp mode cutoff. ${ }^{22}$

(iii). The truncated bare action $S_{\Lambda}$ is of Einstein-Hilbert type with bare couplings $\check{g}_{\Lambda}$ and $\check{\lambda}_{\Lambda}$, respectively. Under these conditions an explicit map relating $\left(g_{k}, \lambda_{k}\right)$ for $k \rightarrow \infty$ to $\left(\check{g}_{\Lambda}, \check{\lambda}_{\Lambda}\right)$ when $\Lambda \rightarrow \infty$ was derived.

This map depends on a parameter $M$ which labels a certain 1-parameter family of measures. This $M$-dependence reflects the fact that it is not the bare action alone which is uniquely determined but rather the combination of measure and bare action: certain redefinitions of the measure can be absorbed by redefinitions of the bare action and vice versa, signaling the "unphysicalness" of the bare action.

The $M$-dependence can be exploited to conveniently adjust the bare coupling constants. In $d=2+\varepsilon$ dimensions there is one particular value of $M$ that leads to an exactly vanishing bare cosmological constant, $\check{\lambda}_{*} \equiv \check{\lambda}_{\Lambda \rightarrow \infty}=0$, and a bare Newton constant $\check{g}_{\Lambda}$ which equals precisely the effective one at the NGFP [29]:

$$
\check{g}_{*}=g_{*} .
$$

After having reconstructed the gravitational functional integral in $d=2+\varepsilon$, we take its 2D limit employing the method of section 3. In combination with eq. (6.1) we obtain

$$
S_{\Lambda}^{\text {grav }}[g]=\frac{(25-N)}{96 \pi} I[g]+\cdots
$$

The dots indicate that there might appear additional terms originating from the zero modes, according to eq. (A.39) in the appendix. Further details about the reconstruction and the derivation of (6.1) will be presented elsewhere [29]. For our present purposes it suffices to know that the bare action has the structure (6.2), displaying a cutoff independent term $\propto I[g]$ and possibly "zero mode terms".

The bare action of the matter system too can be reconstructed according to the results of ref. [12]: for cutoffs satisfying certain constraints the bare action equals precisely the EAA when the respective cutoff scales are identified. Thus, the bare matter action is given by the r.h.s. of eq. (1.3).

We would like to point out that, as a consequence, the number $N$ enters both the matter and the gravitational part of the bare action. (Note that the fixed point value $g_{*}$ depends on $N$, cf. section 3.4.)

\subsection{A functional integral for $2 \mathrm{D}$ asymptotically safe gravity}

(1) The partition function. Based on the above considerations we obtain the full reconstructed partition function

$$
Z=\int[\mathrm{d} \tau] \int \mathcal{D}_{\mathrm{e}^{2 \phi} \hat{g}} \phi Z_{\mathrm{gh}}\left[\mathrm{e}^{2 \phi} \hat{g}\right] Z_{\text {matter }}\left[\mathrm{e}^{2 \phi} \hat{g}\right] Y_{\text {grav }}^{\mathrm{NGFP}}\left[\mathrm{e}^{2 \phi} \hat{g}\right]
$$

\footnotetext{
${ }^{22}$ When the cosmological constant is an issue a higher-derivative regularization in the UV similar to the one in the IR is known to be problematic [9]. For a purely scalar theory without gauge and gravity fields the implications of a sharp cutoff were discussed in ref. [12] recently.
} 
The integrand of (6.3) comprises the following factors: the exponential of the gravitational part of the fixed point action,

$$
Y_{\text {grav }}^{\mathrm{NGFP}}[g] \equiv \exp \left(-\frac{(25-N)}{96 \pi} I[g]+\cdots\right),
$$

the partition function of the matter system,

$$
\begin{aligned}
Z_{\text {matter }}[g] & \equiv \int \mathcal{D} A \exp \left(-\frac{1}{2} \sum_{i=1}^{N} \int \mathrm{d}^{2} x \sqrt{g} g^{\mu \nu} \partial_{\mu} A^{i} \partial_{\nu} A^{i}\right) \\
& =\operatorname{det}^{-N / 2}\left(-\square_{g}\right)=\exp \left(-\frac{N}{96 \pi} I[g]+\cdots\right),
\end{aligned}
$$

the partition function of the $b-c$ ghost system, $Z_{\mathrm{gh}}$, the split symmetry invariant measure for the integration over the Liouville field, $\mathcal{D}_{\mathrm{e}^{2 \phi} \hat{g}} \phi$, and finally the measure $[\mathrm{d} \tau]$ for the integration over the moduli that are implicit in the reference metric pertaining to a given topological type of the spacetime manifold (cf. section 3.1). In eqs. (6.4) and (6.5) we suppressed possible contributions to the bare cosmological constant. Here and in the following we indicate them by the dots.

The behavior under Weyl transformations of the various factors is well known. Using in particular eq. (3.22) with the (non-cosmological constant part of the) renormalized Liouville action, $\Delta I$, as defined in (2.23), we have

$$
\begin{aligned}
Y_{\text {grav }}^{\mathrm{NGFP}}\left[\mathrm{e}^{2 \phi} \hat{g}\right] & =Y_{\text {grav }}^{\mathrm{NGFP}}[\hat{g}] \exp \left(+\frac{(25-N)}{12 \pi} \Delta I[\phi ; \hat{g}]\right), \\
Z_{\text {matter }}\left[\mathrm{e}^{2 \phi} \hat{g}\right] & =Z_{\text {matter }}[\hat{g}] \exp \left(+\frac{N}{12 \pi} \Delta I[\phi ; \hat{g}]\right), \\
Z_{\text {gh }}\left[\mathrm{e}^{2 \phi} \hat{g}\right] & =Z_{\text {gh }}[\hat{g}] \exp \left(+\frac{(-26)}{12 \pi} \Delta I[\phi ; \hat{g}]\right), \\
\mathcal{D}_{\mathrm{e}^{2 \phi} \hat{g}} \phi & =\mathcal{D}_{\hat{g}} \phi \exp \left(+\frac{1}{12 \pi} \Delta I[\phi ; \hat{g}]\right) .
\end{aligned}
$$

As before, possible (measure dependent) terms involving the bare cosmological constant are suppressed in eqs. (6.6). On the r.h.s. of $(6.6 \mathrm{~d}), \mathcal{D}_{\hat{g}} \phi$ is the translational invariant measure now.

Up to this point, the discussion is almost the same as in non-critical string theory [27]. The profound difference lies in the purely gravitational part of the bare action, $Y_{\text {grav }}^{\text {NGFP }}$. Contrary to what happens in any conventional field theory, whose bare action is a postulate rather than the result of a calculation, asymptotically safe gravity in 2 dimensions is based upon a gravitational action which depends explicitly on properties of the matter system. In the example at hand, this dependence is via the number $N$ of $A^{i}$-fields that makes its appearance in the fixed point action and hence in the "Boltzmann factor" (6.4).

(1a) Matter refuses to matter: a compensation mechanism. Remarkably enough, the integrand of (6.3) depends on $N$ only via the product $Z_{\text {matter }} \cdot Y_{\text {grav }}^{\mathrm{NGFP}}$ in which the $N$-dependence cancels between the two factors. Multiplying (6.4) and (6.5) we obtain a 
result which, for any $N$, equals that of pure gravity. It is always the same no matter how many scalar fields there are:

$$
Z_{\text {matter }}[g] Y_{\text {grav }}^{\mathrm{NGFP}}[g]=\exp \left(-\frac{25}{96 \pi} I[g]+\cdots\right)
$$

which transforms as $Z_{\text {matter }}\left[\mathrm{e}^{2 \phi} \hat{g}\right] Y_{\text {grav }}^{\mathrm{NGFP}}\left[\mathrm{e}^{2 \phi} \hat{g}\right]=Z_{\text {matter }}[\hat{g}] Y_{\text {grav }}^{\mathrm{NGFP}}[\hat{g}] \exp \left(+\frac{25}{12 \pi} \Delta I[\phi ; \hat{g}]\right)$ under a Weyl rescaling. As a consequence, the reconstructed functional integral coincides always with that of pure gravity (as long as we do not evaluate the expectation value of observables depending on the $A$ 's and as long as cosmological constant terms do not play a role):

$$
Z=\int[\mathrm{d} \tau] Z_{\text {matter }}[\hat{g}] Y_{\text {grav }}^{\mathrm{NGFP}}[\hat{g}] \int \mathcal{D}_{\mathrm{e}^{2 \phi} \hat{g}} \phi Z_{\mathrm{gh}}\left[\mathrm{e}^{2 \phi} \hat{g}\right] \exp \left(+\frac{25}{12 \pi} \Delta I[\phi ; \hat{g}]+\cdots\right) .
$$

(1b) Zero total central charge. Over and above the specific form of its matter dependence, the fixed point action displays a second miracle: its central charge equals precisely the critical value 25 . Up to a cosmological constant term possibly, this leads to a complete cancellation of the entire $\phi$-dependence of the integrand once the ghost contribution (6.6c) and the "Jacobian" factor in $(6.6 \mathrm{~d})$ are taken into account:

$$
Z=\int[\mathrm{d} \tau] Z_{\text {gh }}[\hat{g}] Z_{\text {matter }}[\hat{g}] Y_{\text {grav }}^{\mathrm{NGFP}}[\hat{g}] \int \mathcal{D}_{\hat{g}} \phi \exp (0+\cdots) .
$$

Hence, for every choice of the matter sector, the total system described by the reconstructed functional integral of asymptotically safe $2 \mathrm{D}$ gravity is a conformal field theory with central charge zero. The various sectors of this system contribute to the total central charge as follows:

$$
c_{\text {tot }}=\underbrace{(25-N)}_{\text {NGFP, grav. part }}+\underbrace{N}_{\text {matter }}+\underbrace{1}_{\text {Jacobian }}+\underbrace{(-26)}_{\text {ghosts }}=0 .
$$

Actually the result (6.10) is even more general than we indicated so far. In addition to the scalar matter fields underlying our considerations up to this point we can also bring massless free Dirac fermions into play and couple them (minimally) to the dynamical metric by adding a corresponding term to the matter action (1.3). The contribution of each of such fermions to the $\beta$-function of Newton's constant in $d=2+\varepsilon$ is the same as for a scalar field [90, 91], that is, fermions and scalars enter the central charge in the same way. Hence, in all above equations for $\beta$-functions and central charges we may identify $N$ with

$$
N \equiv N_{\mathrm{S}}+N_{\mathrm{F}}
$$

where $N_{\mathrm{S}}$ and $N_{\mathrm{F}}$ denote the number of real scalars and Dirac fermions, respectively. In particular, we recover the same cancellation in the total central charge as in eq. (6.10): the central charge of the matter system, $+N$, removes exactly a corresponding piece in the pure gravity contribution enforced by the fixed point, $25-N$. 
(2) Observables. By inserting appropriate functions $\overline{\mathscr{O}}[\phi, A ; \hat{g}]$ into the path integral (6.3) we can in principle evaluate the expectation values of arbitrary observables $\mathscr{O}[g, A]=\mathscr{O}\left[\mathrm{e}^{2 \phi} \hat{g}, A\right]$. In the case when the observables do not involve the matter fields, their expectation values read

$$
\langle\mathscr{O}\rangle=\frac{1}{Z} \int[\mathrm{d} \tau] Z_{\text {matter }}[\hat{g}] Y_{\text {grav }}^{\mathrm{NGFP}}[\hat{g}] \int \mathcal{D}_{\mathrm{e}^{2 \phi} \hat{g}} \phi Z_{\text {gh }}\left[\mathrm{e}^{2 \phi} \hat{g}\right] \overline{\mathscr{O}}[\phi ; \hat{g}] \exp \left(\frac{25}{12 \pi} \Delta I[\phi ; \hat{g}]\right) .
$$

Without actually evaluating the $\phi$-integral we see that when the cosmological constant term is negligible the expectation value of purely gravitational observables does not depend on the presence or absence of matter and its properties, provided the background factor $Z_{\text {matter }}[\hat{g}]$ in (6.12) cancels against the corresponding piece in the denominator of (6.12). At the very least, this happens if one considers expectation values at a fixed point of moduli space.

(3) Gravitational dressing. As it is well known [92-94], it is not completely straightforward to find the functional $\overline{\mathscr{O}}[\phi ; \hat{g}]$ which one must use under a conformally gauge-fixed path integral in order to represent a given diffeomorphism (and, trivially, Weyl) invariant observable $\mathscr{O}[g]=\mathscr{O}\left[\mathrm{e}^{2 \phi} \hat{g}\right]$. The association $\mathscr{O} \rightarrow \overline{\mathscr{O}}$ should respect the following conditions [94]: $\overline{\mathscr{O}}[\phi ; \hat{g}]$ must be invariant under diffeomorphisms, it must approach $\mathscr{O}\left[\mathrm{e}^{2 \phi} \hat{g}\right]$ in the classical limit and $\mathscr{O}[\hat{g}]$ in the limit $\phi \rightarrow 0$, and most importantly it must be such that the expectation value computed with its help is independent of the reference metric chosen, $\hat{g}_{\mu \nu}$.

Let us briefly recall the David-Distler-Kawai (DDK) solution to this problem [92, 93]. For this purpose we consider 2D gravity coupled to an arbitrary matter system described by a CFT with central charge $c$ and partition function $Z_{\mathrm{M}}^{(c)}[g]$. First we want to evaluate the partition function for a fixed volume (area) of spacetime, $V$ :

$$
Z_{V}=\int \frac{\mathcal{D} g}{\operatorname{vol}(\text { Diff })} Z_{\mathrm{M}}^{(c)}[g] \delta\left(V-\int \mathrm{d}^{2} x \sqrt{g}\right) .
$$

This integral involves the observable $\mathscr{O}[g] \equiv \int \mathrm{d}^{2} x \sqrt{g} \equiv \int \mathrm{d}^{2} x \sqrt{\hat{g}} \exp (2 \phi)$. The associated $\overline{\mathscr{O}}$ satisfying the above conditions turns out to require only a "deformation" of the prefactor of $\phi$ in the exponential:

$$
\overline{\mathscr{O}}[\phi ; \hat{g}]=\int \mathrm{d}^{2} x \sqrt{\hat{g}} \exp \left(2 \alpha_{1} \phi\right) .
$$

The modified prefactor $\alpha_{1}$ depends on the central charge of the matter CFT according to

$$
\alpha_{1}=\frac{2 \sqrt{25-c}}{\sqrt{25-c}+\sqrt{1-c}}=\frac{1}{12}[25-c-\sqrt{(25-c)(1-c)}] .
$$

Thus, in the conformal gauge, $Z_{V}$ reads as follows:

$$
Z_{V}=\int[\mathrm{d} \tau] Z_{\mathrm{gh}}[\hat{g}] Z_{\mathrm{M}}^{(c)}[\hat{g}] \int \mathcal{D}_{\hat{g}} \phi \delta\left(V-\int \mathrm{d}^{2} x \sqrt{\hat{g}} \mathrm{e}^{2 \alpha_{1} \phi}\right) \exp \left(-\frac{(25-c)}{12 \pi} \Delta I[\phi ; \hat{g}]\right) .
$$

Similarly the expectation value of an arbitrary observable $\mathscr{O}[g]$ at fixed volume is given by $\langle\mathscr{O}[g]\rangle=Z_{V}^{-1}\langle\overline{\mathscr{O}}[\phi ; \hat{g}]\rangle^{\prime}$. Here $\langle\cdots\rangle^{\prime}$ is defined by analogy with (6.16) but with the additional factor $\overline{\mathscr{O}}[\phi ; \hat{g}]$ under the $\phi$-integral. 
The DDK approach to the gravitational dressing of operators from the matter sector was developed as a conformal gauge-analogue to the work of Knizhnik, Polyakov and Zamolodchikov (KPZ) $[95,96]$ based upon the light cone gauge.

To study gravitational dressing, let us consider an arbitrary spinless primary field $\mathscr{O}_{n}[g] \equiv \int \mathrm{d}^{2} x \sqrt{g} \mathcal{P}_{n+1}(g)$, where $\mathcal{P}_{n}(g)$ is a generic scalar involving the matter fields with conformal weight $(n, n)$, that is, it responds to a rescaling of the metric according to $\mathcal{P}_{n}\left(\mathrm{e}^{-2 \sigma} g\right)=\mathrm{e}^{2 n \sigma} \mathcal{P}_{n}(g)$. Under the functional integral, the observables $\mathscr{O}_{n}$ are then represented by

$$
\overline{\mathscr{O}}_{n}[\phi ; \hat{g}]=\int \mathrm{d}^{2} x \sqrt{\hat{g}} \exp \left(2 \alpha_{-n} \phi\right) \mathcal{P}_{n+1}(\hat{g}),
$$

where the $c$-dependent constants in the dressing factors generalize eq. (6.15):

$$
\alpha_{n}=\frac{2 n \sqrt{25-c}}{\sqrt{25-c}+\sqrt{25-c-24 n}}
$$

Using (6.18) it is straightforward now to write down the modified conformal dimensions corrected by the quantum gravity effects.

The results of the DDK approach reproduce those of KPZ (valid for spherical topology) and generalize them for spacetimes of arbitrary topology. Within the framework of the EAA and its functional RG equations, the KPZ relations were derived from Liouville theory in ref. [28]; for a review see [22].

(4) Quenching of the KPZ scaling. Let us apply the general DDK-KPZ formulae to the NGFP theory of asymptotically safe gravity. We must replace then

$$
c \longrightarrow c_{\text {grav }}^{\mathrm{NGFP}}+N \equiv(25-N)+N=25,
$$

since the relevant bare action arises now from both the integrated-out matter fluctuations and the pure-gravity NGFP contribution, $Y_{\text {grav }}^{\text {NGFP }}$. Setting $c=25$ in eqs. (6.15) and (6.18) we obtain

$$
\alpha_{1}=0 \quad \text { and } \quad \alpha_{n}=0,
$$

respectively. This implies that the Liouville field completely decouples from the area operator (6.14) and any of the observables (6.17).

As a consequence, the dynamics of the matter system is unaffected by its coupling to quantum gravity. In particular, its critical behavior is described by the properties (critical exponents, etc.) of the matter CFT defined on a non-dynamical, rigid background spacetime. Thus, the specific properties of the NGFP lead to a perfect "quenching" of the a priori expected KPZ scaling.

(5) Relation to non-critical string theory. The functional integral (6.9) is identical to the partition function of non-critical string theory in 25 Euclidean dimensions. This theory is equivalent to the usual critical bosonic string living in a $(25+1)$-dimensional Minkowski space whereby the Liouville mode plays the role of the time coordinate in the target space [97-99]. Whether we consider pure asymptotically safe gravity in two dimensions, or couple any number of scalar and fermionic matter fields to it, the resulting 
partition function equals always the one induced by the fluctuations of precisely 25 string positions $X^{m}\left(x^{\mu}\right)$.

There is, however, a certain difference between asymptotically safe gravity and noncritical string theory in the way the special case of vanishing total central charge, i.e. of precisely 25 target space dimensions, is approached. To see this note that in the present paper we related the Liouville field to the metric by eq. (3.37), and at no point we redefined $\phi$ by absorbing any constant factors in it. In this connection the Liouville action for a general central charge $c$ has the structure $\Gamma_{k}^{\mathrm{L}}=-\frac{c}{24 \pi} \int\left(\hat{D}_{\mu} \phi \hat{D}^{\mu} \phi+\hat{R} \phi\right)+\cdots$.

(i). In order to combine $\Gamma_{k}^{\mathrm{L}}$ with the action of the string positions, $+\frac{1}{8 \pi} \int \hat{D}_{\mu} X^{m} \hat{D}^{\mu} X^{m}$, it is natural to introduce the redefined field

$$
\phi^{\prime} \equiv Q \phi \quad \text { with } \quad Q \equiv \sqrt{\frac{c}{3}}
$$

in terms of which $\Gamma_{k}^{\mathrm{L}}=-\frac{1}{8 \pi} \int\left(\hat{D}_{\mu} \phi^{\prime} \hat{D}^{\mu} \phi^{\prime}+Q \hat{R} \phi^{\prime}\right)+\cdots$. It is this new field $\phi^{\prime}$ that plays the role of time in target space and combines with the $X^{m}$ 's in the conventionally normalized action $\frac{1}{8 \pi} \int\left(-\hat{D}_{\mu} \phi^{\prime} \hat{D}^{\mu} \phi^{\prime}+\hat{D}_{\mu} X^{m} \hat{D}^{\mu} X^{m}-Q \hat{R} \phi^{\prime}\right)+\cdots$ which enhances the original $\mathrm{O}(25)$ symmetry to the full Lorentz group in target space, $\mathrm{O}(1,25)$ [99].

In string theory conformal invariance requires the total central charge to vanish, $c_{\text {tot }}=0$. Hence, arguing that the combined $\left(X^{0} \equiv \phi^{\prime}, X^{m}\right)$-quantum system is equivalent to the usual bosonic string theory in the critical dimension involves taking the limit $c \equiv c_{\text {tot }} \rightarrow 0$ in the above formulae. Obviously this requires some care in calculating correlation functions as the relationship $\phi^{\prime} \equiv \sqrt{c / 3} \phi$ breaks down in this limit. Considering vertex operators for the emission of a tachyon of 26-dimensional momentum $\left(P_{0}, P_{m}\right)$, say, this involves combining the rescaling $\phi \rightarrow \sqrt{c / 3} \phi$ with a corresponding rescaling of $P_{0}$ with the inverse factor, $P_{0} \rightarrow \sqrt{3 / c} P_{0}$, rendering their product $P_{0} X^{0} \equiv P_{0} \phi^{\prime}$ independent of $c$. The vertex operator $\exp \left\{i\left(-P_{0} X^{0}+P_{m} X^{m}\right)\right\}$, too, displays the full $\mathrm{O}(1,25)$ invariance. (See $[97,98]$ for a detailed discussion.)

(ii). In 2D asymptotically safe quantum gravity, too, the total central charge was found to vanish, albeit for entirely different reasons than in string theory. However, here there is no obvious reason or motivation for any rescaling before letting $c \rightarrow 0$. In all of the above equations, including (6.14) and (6.17), $\phi$ still denotes the Liouville field introduced originally. In quantum gravity we let $c \rightarrow 0$ in the most straightforward way, setting in particular $c=0$ directly in (6.15) and (6.18). This is what led us to (6.20), that is, the disappearance of $\phi$ from the exponentials $\exp \left(2 \alpha_{-n} \phi\right)$ multiplying the matter operators and the "quenching" of the KPZ-scaling.

\subsection{Comparison with Monte Carlo results}

In earlier work [100-102] indications were found that suggest that Quantum Einstein Gravity in the continuum formulation based upon the EAA might be related to the discrete approach employing Causal Dynamical Triangulation [79, 103]. In particular, the respective predictions for the fractal dimensions of spacetime were compared in detail and turned out similar $[101,102]$. It is therefore natural to ask whether the quenching 
of the KPZ-scaling due to the above compensation mechanism can be seen in 2D CDT simulations. And in fact, the Monte-Carlo studies indeed seem to suggest a picture that looks quite similar at first sight: coupling several copies of the Ising model [104] or the Potts model [105] to 2-dimensional Lorentzian quantum gravity in the CDT framework, there is strong numerical evidence that the critical behavior of the combined system, in the matter sector, is described by the same critical exponents as on a fixed, regular lattice. Under the influence of the quantum fluctuations in the geometry the critical exponents do not get shifted to their KPZ values.

While this seems a striking confirmation of our Asymptotic Safety-based prediction, one should be careful in interpreting these results. In particular, it is unclear whether the underlying physics is the same in both cases. In CDT, the presence (absence) of quantum gravity corrections of the matter exponents is attributed to the presence (absence) of baby universes in Euclidean (causal Lorentzian) dynamical triangulations. In our approach instead, the quantum gravity corrections that could in principle lead to the KPZ exponents are exactly compensated by the explicit matter dependence of the pure gravity-part in the bare action. This matter dependence is an immediate consequence of the very Asymptotic Safety requirement.

As yet we considered conformal matter only which was exemplified by massless, minimally coupled scalar fields. In the non-conformal case when those fields are given a mass for instance, the compensation between the matter contributions to the bare NGFP action and those resulting from integrating them out will in general no longer be complete. On the EAA side, this situation is described by a trajectory $k \mapsto \Gamma_{k}$ that runs away from the fixed point as $k$ decreases, and typically the resulting ordinary effective action of the gravity + matter system, $\Gamma_{k=0}$, will indeed be affected by the presence of matter.

This expected behavior seems to be matched by the results of very recent $2 \mathrm{D}$ Monte-Carlo simulations of CDT coupled to more than one massive scalar field [106-108]. It was found that, above a certain value of their mass, the dynamics of the CDT+matter system is significantly different from the massless case. In particular, a characteristic "blob + stalk" behavior was observed, well known from 4D pure gravity CDT simulations, but absent in $2 \mathrm{D}$ with conformal matter.

\section{Conclusions}

In this paper we started from the Einstein-Hilbert truncation for the effective average action of metric quantum gravity in $d>2$ dimensions and constructed its intrinsically 2 -dimensional limit. Contrary to earlier work on the $\varepsilon$-expansion of $\beta$-functions this limit was taken directly at the level of the action functional. We saw that it turns the (local, second-derivative) Einstein-Hilbert term into the non-local Polyakov action.

Using this result we were able to conclude that in 2D the non-Gaussian fixed point underlying Asymptotic Safety gives rise to a unitary conformal field theory whose gravitational sector possesses the central charge +25 . We analyzed the properties of the fixed point CFT using both a gauge invariant description and a calculation based on the conformal gauge where it is represented by a Liouville theory. 
Finding that the complete fixed point action amounts to a CFT with vanishing total central charge, we compared and contrasted asymptotically safe quantum gravity in 2 dimensions with non-critical string theory.

Furthermore, exploring the gravitational dressing in 2D asymptotically safe gravity coupled to conformal matter we discovered a rather surprising compensation mechanism that leads to a complete quenching of the KPZ-scaling, that is, of the behavior we would have expected to occur a priori. Remarkably enough, this quenching is precisely what is observed in Monte-Carlo simulations of analogous systems in the framework of causal dynamical triangulation. We argued that these observations can possibly be interpreted as a reflection of the compensating effect displayed by the integrated-out matter fluctuations and the explicit dependence of the bare gravitational action on properties of the matter sector.

We close with a number of further comments.

(1). An important step in proving the viability of the Asymptotic Safety program consists in demonstrating that Hilbert space positivity can be achieved together with Background Independence and the nonperturbative renormalizability. While we consider our present result on the unitarity of the pertinent CFT as an encouraging first insight, it is clear, however, that the $2 \mathrm{D}$ case is not yet a crucial test since the gravitational field has no independent degrees of freedom, and so there is no pure-gravity subspace of physical states whose positivity would be at stake. To tackle the higher dimensional case additional techniques will have to be developed. Nevertheless, it is interesting that at least at the purely geometric level the remarkable link between the Einstein-Hilbert and the Polyakov action which we exploited has an analogue in all even dimensions $d=2 n$. Each nontrivial cocycle of the Weyl cohomology yields, in an appropriate limit $d \rightarrow 2 n$, a well-defined nonlocal action that is known to be part of the standard effective action in $2 n$ dimensions [43].

(2). A number of general lessons we learned here will be relevant in higher dimensions, too. We mention in particular that the issue of unitarity cannot be settled by superficially checking for the stability of some bare action and ruling out "wrong sign" kinetic terms as this is implied sometimes. We saw that the CFT which is at the heart of the NGFP is unitary even though in conformal gauge it entails a negative kinetic energy of the Liouville field. As we explained in section 4, the background field, indispensable in our approach to quantum gravity, plays an important role in reconciling these properties.

(3). We determined the crucial central charge $c_{\mathrm{grav}}^{\mathrm{NGFP}}$ from the leading term in the $\beta$ function of Newton's constant, and we saw that depending on which parametrization of the metric is chosen the pure gravity result is either 25 or 19 for the exponential and the linear parametrization, respectively. In section 5 we found convincing evidence for accepting the result of the former, +25 , as the correct one in the present context. Nevertheless, the issue of parametrization dependence is not fully settled yet, and one should still be open towards the possibility that the two sets of results, obtained from the same truncation ansatz but different choices of the fluctuating field, actually might refer to different universality classes.

(4). Regarding different universality classes, it is perhaps not a pure coincidence that the "19" is also among the "critical dimensions for non-critical strings" which were found by 
Gervais [109-114]:

$$
D_{\text {crit }}=7,13,19 \text {. }
$$

They correspond to gravitational central charges $c_{\text {grav }}=19,13,7$, respectively. For these special values the Virasoro algebra admits a unitary truncation, that is, there exists a subspace of the usual state space on which a corresponding chiral algebra closes, and which is positive (in the sense that it contains no vectors $|\psi\rangle$ with $\langle\psi \mid \psi\rangle<0$ ). The associated string theories were advocated as consistent extensions of standard Liouville theory, which is valid only for $c<1$ and $c>25$ when gravity is weakly coupled, into the strongly coupled regime, $1<c<25$, in which the KPZ formulae would lead to meaningless complex answers.

Thus, for the time being, we cannot exclude the possibility that a better understanding of the RG flow computed with the linear parametrization (but with more general truncations than those analyzed in the present paper) will lead to the picture that there exists a second pure gravity fixed point compatible with Hilbert space positivity, namely at $c_{\text {grav }}=19$, and that this fixed point represents another, inequivalent universality class.

We know already that this picture displays the following correlation between parametrization and universality class, which we would then indeed consider the natural one: the exponential parametrization, i.e. the "conservative" one in the sense that it covers only nonzero, nondegenerate, hence "more classical" metrics having a fixed signature, leads to $c_{\text {grav }}=25$ which is located just at the boundary of the strong coupling interval. In the way it is employed, the linear parametrization, instead, gives rise to an integration also over degenerate, even vanishing tensor field configurations not corresponding to any classical metric; typically enough, it is this parametrization that would be linked to the hypothetical, certainly quite non-classical theory with $c_{\text {grav }}=19$ deep in the strong coupling domain.

Whatever the final answer will be it seems premature, also in more than 2 dimensions, to regard the exponential parametrization merely as a tool to do calculations in a more precise or more convenient way than this would be possible with the linear one. It might rather be that in this manner we are actually computing something else.

\section{Acknowledgments}

We are grateful to J. Ambjørn, J. Gizbert-Studnicki, R. Loll, C. Pagani and R. Percacci for helpful discussions.

\section{A Weyl transformations, zero modes and the induced gravity action}

In this appendix we list the behavior of various geometric objects under Weyl transformations, including the induced gravity functional, which is needed in the main part of this paper. Weyl transformations are given by

$$
g_{\mu \nu}=\mathrm{e}^{2 \sigma} \hat{g}_{\mu \nu},
$$

where $\sigma$ is a scalar function on the spacetime manifold. 
(1). From the definition of the Christoffel connection we immediately obtain

$$
\Gamma_{\mu \nu}^{\alpha}=\hat{\Gamma}_{\mu \nu}^{\alpha}+\delta_{\mu}^{\alpha} \hat{D}_{\nu} \sigma+\delta_{\nu}^{\alpha} \hat{D}_{\mu} \sigma-\hat{g}_{\mu \nu} \hat{D}^{\alpha} \sigma .
$$

Note that indices (on the right hand side) are raised and lowered by means of $\hat{g}^{\mu \nu}$ and $\hat{g}_{\mu \nu}$, respectively. From (A.2) we easily deduce the Riemann tensor and its contractions,

$$
\begin{aligned}
R_{\mu \nu \rho}^{\alpha}= & \hat{R}_{\mu \nu \rho}^{\alpha}+2 \hat{g}_{\mu[\nu} \hat{D}_{\rho]} \hat{D}^{\alpha} \sigma-2 \delta_{[\nu}^{\alpha} \hat{D}_{\rho]} \hat{D}_{\mu} \sigma-2 \hat{g}_{\mu[\nu} \hat{D}_{\rho]} \sigma \hat{D}^{\alpha} \sigma \\
& +2 \delta_{[\nu}^{\alpha} \hat{D}_{\rho]} \sigma \hat{D}_{\mu} \sigma+2 \hat{g}_{\mu[\nu} \delta_{\rho]}^{\alpha} \hat{D}_{\beta} \sigma \hat{D}^{\beta} \sigma \\
R_{\mu \nu}= & \hat{R}_{\mu \nu}-(d-2)\left(\hat{D}_{\mu} \hat{D}_{\nu} \sigma-\hat{D}_{\mu} \sigma \hat{D}_{\nu} \sigma\right)-\hat{g}_{\mu \nu}\left[\hat{\square} \sigma+(d-2) \hat{D}_{\alpha} \sigma \hat{D}^{\alpha} \sigma\right], \\
R= & \mathrm{e}^{-2 \sigma}\left[\hat{R}-(d-1)(d-2) \hat{D}_{\mu} \sigma \hat{D}^{\mu} \sigma-2(d-1) \hat{\square} \sigma\right],
\end{aligned}
$$

where $\hat{\square} \equiv \hat{D}_{\alpha} \hat{D}^{\alpha}$ and the square brackets enclosing indices denote antisymmetrization, $A_{[\mu \nu]}=\frac{1}{2}\left(A_{\mu \nu}-A_{\nu \mu}\right)$. Note that since the underlying connection is the Christoffel symbol, i.e. it is torsion free, we have $\hat{D}_{\mu} \hat{D}_{\nu} \sigma=\hat{D}_{\nu} \hat{D}_{\mu} \sigma$. For the Einstein tensor we have

$$
G_{\mu \nu}=\hat{G}_{\mu \nu}+(d-2)\left[-\hat{D}_{\mu} \hat{D}_{\nu} \sigma+\hat{g}_{\mu \nu} \hat{\square} \sigma+\hat{D}_{\mu} \sigma \hat{D}_{\nu} \sigma+\frac{d-3}{2} \hat{g}_{\mu \nu} \hat{D}_{\alpha} \sigma \hat{D}^{\alpha} \sigma\right] .
$$

Furthermore, the metric determinant transforms as

$$
\sqrt{g}=\sqrt{\hat{g}} \mathrm{e}^{d \sigma} .
$$

Hence, we arrive at the useful relations

$$
\begin{aligned}
\sqrt{g} R & =\mathrm{e}^{(d-2) \sigma} \sqrt{\hat{g}}\left[\hat{R}-(d-1)(d-2) \hat{D}_{\mu} \sigma \hat{D}^{\mu} \sigma-2(d-1) \hat{\square} \sigma\right] \\
\int \mathrm{d}^{d} x \sqrt{g} R & =\int \mathrm{d}^{d} x \sqrt{\hat{g}} \mathrm{e}^{(d-2) \sigma}\left[\hat{R}+(d-1)(d-2) \hat{D}_{\mu} \sigma \hat{D}^{\mu} \sigma\right] .
\end{aligned}
$$

The transformation behavior of the Laplacian is given by

$$
\square f=\mathrm{e}^{-2 \sigma} \hat{\square} f+(d-2) \mathrm{e}^{-2 \sigma} \hat{D}_{\mu} \sigma \hat{D}^{\mu} \sigma,
$$

where $f$ is an arbitrary scalar function.

(2). In the special case of two dimensions, $d=2$, we obtain

$$
\begin{aligned}
R & =\mathrm{e}^{-2 \sigma}[\hat{R}-2 \hat{\square} \sigma], \\
\sqrt{g} R & =\sqrt{\hat{g}}[\hat{R}-2 \hat{\square} \sigma], \\
\square f & =\mathrm{e}^{-2 \sigma} \hat{\square} f .
\end{aligned}
$$

(3). Due to its relevance for the induced gravity action we are particularly interested in the transformation behavior of $\square^{-1} R$, with the inverse Laplacian (Green function) $\square^{-1} \equiv$ $\square^{-1}(x, y)$, where $\left(\square^{-1} R\right)(x)$ refers to

$$
\left(\square^{-1} R\right)(x)=\int \mathrm{d}^{d} y \sqrt{g} \square^{-1}(x, y) R(y) .
$$


If $\square$ has no zero modes, its inverse is defined by $\square\left[\square^{-1}(x, y)\right]=\frac{1}{\sqrt{g}} \delta(x-y)$. On the other hand, if $\square$ has normalizable zero modes, then $\square^{-1}$ is defined as the inverse of $\square$ on the orthogonal complement to its kernel, where the delta function has to be modified appropriately, that is, $\square \square^{-1}(x, y)=\frac{1}{\sqrt{g}} \delta(x-y)-\operatorname{Pr}_{0}(x, y)$ where $\operatorname{Pr}_{0}$ denotes the projection onto zero modes. Whenever we write $\square^{-1}$ in this article, this definition is meant implicitly.

(4). Since the consideration of zero modes requires a more careful treatment, we first consider the situation where zero modes are absent in the following subsection, before investigating the general case in subsection A.2.

\section{A.1 The induced gravity action in the absence of zero modes}

If the Laplacian has no zero modes, then the equation $\square f=h$ can be solved for $f$ by direct inversion of $\square$, that is, $f=\square^{-1} h$. In this case the transformation behavior of the Green function $\square^{-1}$ is given by

$$
\square^{-1}\left(\mathrm{e}^{-2 \sigma} h\right)=\hat{\square}^{-1} h .
$$

This gives rise to

$$
\square^{-1} R=\hat{\square}^{-1} \hat{R}-2 \sigma .
$$

For our arguments in section 3.3 we need to determine the transformation behavior of the induced gravity functional $I[g]$ which can be defined as the normalized finite part of Polyakov's induced effective action [27]

$$
\Gamma_{\text {ind }}[g]=\frac{1}{2} \operatorname{Tr} \ln (-\square) .
$$

In the absence of zero modes the trace in (A.17) can be computed explicitly. The result, $\Gamma_{\text {ind }}[g]$, consists of a universal finite part and a regularization scheme dependent divergent part. Regularizing by means of a proper time cutoff [115-118], for instance, one obtains from eq. (A.17):

$$
\Gamma_{\text {ind }}[g]=\frac{1}{96 \pi} \int \mathrm{d}^{2} x \sqrt{g} R \square^{-1} R-\frac{1}{8 \pi s} \int \mathrm{d}^{2} x \sqrt{g} .
$$

The second term on the r.h.s. of eq. (A.18) is scheme dependent and divergent in the limit $s \rightarrow 0$. It might be absorbed by a redefinition of the cosmological constant. The first term, on the other hand, contains all relevant information, so we focus on it for our further investigations. We define the induced gravity functional $I[g]$ to be proportional to the finite part of $\Gamma_{\text {ind }}[g]$,

$$
\left.I[g] \equiv 96 \pi \Gamma_{\text {ind }}[g]\right|_{\text {finite }}=\int \mathrm{d}^{2} x \sqrt{g} R \square^{-1} R .
$$

Using (A.12) and (A.16) we now obtain, after integrating by parts,

$$
I[g]=\int \mathrm{d}^{2} x \sqrt{\hat{g}}\left[\hat{R} \hat{\square}^{-1} \hat{R}-4 \hat{R} \sigma+4 \sigma \hat{\square} \sigma\right] .
$$

This can be written as

$$
I[g]-I[\hat{g}]=-8 \Delta I[\sigma ; \hat{g}],
$$


with the functional $\Delta I$ defined by

$$
\Delta I[\sigma ; \hat{g}] \equiv \frac{1}{2} \int \mathrm{d}^{2} x \sqrt{\hat{g}}\left[\hat{D}_{\mu} \sigma \hat{D}^{\mu} \sigma+\hat{R} \sigma\right] .
$$

These results prove useful for calculating the 2D limit of the Einstein-Hilbert action, as applied in sections 3.2 and 3.3.

\section{A.2 The treatment of zero modes}

What is different and which results of section A.1 remain valid when the scalar Laplacian has one or more zero modes? To illustrate the issue let us start from scratch and consider a functional integral over a simple scalar field $X$ minimally coupled to the metric. Integrating out $X$ will "induce" a gravity action for the metric then. The corresponding partition function is given by

$$
\tilde{Z}[g] \equiv \int \mathcal{D} X \mathrm{e}^{-\frac{1}{2} \int \mathrm{d}^{2} x \sqrt{g} g^{\mu \nu} \partial_{\mu} X \partial_{\nu} X}=\int \mathcal{D} X \mathrm{e}^{-\frac{1}{2} \int \mathrm{d}^{2} x \sqrt{g} X(-\square) X} .
$$

(The notation with the tilde is chosen since definition (A.23) is pathological and gets modified as shown in the following.) Let us expand the field $X$ in terms of normalized eigenmodes $\varphi^{(n)}$ of the Laplacian $-\square$, that is, $X=\sum_{n} c_{n} \varphi^{(n)}$, where $-\square \varphi^{(n)}=\lambda_{n} \varphi^{(n)}$, with the normalization $\int \mathrm{d}^{2} x \sqrt{g} \varphi^{(n)}(x) \varphi^{(m)}(x)=\delta_{m n}$. Then the integral in (A.23) can be written as

$$
\tilde{Z}[g]=\int \prod_{n} \frac{\mathrm{d} c_{n}}{\sqrt{2 \pi}} \mathrm{e}^{-\frac{1}{2} \sum_{n} \lambda_{n} c_{n}^{2}}
$$

Now let us suppose that the Laplacian has a zero mode, $-\square \varphi^{(0)}=0$, i.e. $\lambda_{0}=0$. In this case the integration over its Fourier coefficient, $\int \mathrm{d} c_{0} \mathrm{e}^{-\frac{1}{2} \lambda_{0} c_{0}^{2}}=\int \mathrm{d} c_{0} 1$, is divergent, and so is $\tilde{Z}[g]$. Thus, the zero mode(s) has to be excluded from the path integral in the first place. The correct definition reads

$$
Z[g] \equiv \int \mathcal{D}^{\prime} X \mathrm{e}^{-\frac{1}{2} \int \mathrm{d}^{2} x \sqrt{g} g^{\mu \nu} \partial_{\mu} X \partial_{\nu} X} .
$$

Here and in the following the prime denotes the exclusion of zero modes.

We will consider only connected manifolds with vanishing boundary. In that case the Laplacian has (at most) one single normalized zero mode. It is given by

$$
\varphi^{(0)}=1 / \sqrt{V},
$$

with the volume, or area, $V=\int \mathrm{d}^{2} x \sqrt{g}$.

Performing the Gaussian integrals in eq. (A.25) one obtains

$$
Z[g]=\left[\operatorname{det}^{\prime}(-\square)\right]^{-\frac{1}{2}} .
$$

The corresponding effective action $\Gamma_{\text {ind }}$ is determined by $Z \equiv \mathrm{e}^{-\Gamma_{\text {ind }}}$, leading to

$$
\Gamma_{\text {ind }}[g]=\frac{1}{2} \ln \operatorname{det}^{\prime}(-\square)=\frac{1}{2} \operatorname{Tr}^{\prime} \ln (-\square)
$$


which is Polyakov's induced gravity action, adapted to taking account of zero modes. In order to find an integral representation for $\Gamma_{\text {ind }}$ similar to eq. (A.18) it turns out convenient to consider the variation of $\Gamma_{\text {ind }}$ under a finite Weyl transformation, giving rise to a strictly local term and a term involving the logarithm of the volume (see e.g. [119]): the finite part of the variation reads

$$
\Gamma_{\text {ind }}[g]-\Gamma_{\text {ind }}[\hat{g}]=-\frac{1}{12 \pi} \Delta I[\sigma ; \hat{g}]+\frac{1}{2} \ln (V / \hat{V}),
$$

with the volume terms $V \equiv \int \mathrm{d}^{2} x \sqrt{g}$ and $\hat{V} \equiv \int \mathrm{d}^{2} x \sqrt{\hat{g}}$, and with $\Delta I[\sigma ; \hat{g}]$ as defined in eq. (A.22). The second term on the r.h.s. of (A.29) originates from the zero mode contribution contained in the conformal factor.

To extract from (A.29) an explicit expression for $\Gamma_{\text {ind }}$ that depends only on one metric, we would like to eliminate the conformal factor and rewrite also the r.h.s. of (A.29) as the difference between some functional evaluated at $g$ and the same functional evaluated at $\hat{g}$. Although the existence of such a representation can be proven [120], the explicit form of $\Gamma_{\text {ind }}[g]$ with only one argument is (to the best of our knowledge) not known in general. As already pointed out in ref. [121], the problem occurs for uniform rescalings when the conformal factor is a constant, i.e. proportional to the zero mode: in this case even the formula $\int g^{\mu \nu} \frac{\delta S[g]}{\delta g_{\mu \nu}}=\left.\frac{1}{2} \frac{\partial S\left[\mathrm{e}^{2 \sigma} g\right]}{\partial \sigma}\right|_{\sigma=0}$, where $\sigma$ is a constant, does not apply, a counterexample being the induced gravity functional (A.19) which is invariant under uniform rescalings but whose metric variation gives rise to the anomaly proportional to $R$.

To eliminate the conformal factor in (A.29) we would like to solve the equation

$$
\square \sigma=\frac{1}{2 \sqrt{g}}(\sqrt{\hat{g}} \hat{R}-\sqrt{g} R)
$$

for $\sigma$, where (A.30) follows from (A.12) and the identity $\sqrt{\hat{g}} \hat{\square}=\sqrt{g} \square$, valid in 2D. The existence of a solution is guaranteed by the fact that the r.h.s. of (A.30) is orthogonal to the zero mode, thanks to topological invariance. However, the conformal factor itself could have a contribution from the zero mode. As a consequence, the solution for $\sigma$ is not unique. Employing the Green function $\square^{-1}$ as defined below eq. (A.14) we obtain

$$
\sigma=\frac{1}{2} \square^{-1} \frac{1}{\sqrt{g}}(\sqrt{\hat{g}} \hat{R}-\sqrt{g} R)+\frac{1}{V} \int \sqrt{g} \sigma,
$$

where the second term is the constant zero mode part. (Recall that $\square^{-1}$ is the inverse of $\square$ on the orthogonal complement to the kernel of $\square$, and it satisfies $\square \square^{-1}(x, y)=$ $\frac{1}{\sqrt{g}} \delta(x-y)-\frac{1}{V}$.) Making use of the relation $\sigma=\frac{1}{2} \ln (\sqrt{g} / \sqrt{\hat{g}})$ the last term in (A.31) can be expressed in terms of the metrics $g_{\mu \nu}$ and $\hat{g}_{\mu \nu}$, too. Then eq. (A.29) becomes

$$
\Gamma_{\text {ind }}[g]-\Gamma_{\text {ind }}[\hat{g}]=\Gamma_{\text {ind }}[g, \hat{g}]
$$

with the both $g_{\mu \nu^{-}}$and $\hat{g}_{\mu \nu}$-dependent functional [120]

$$
\begin{aligned}
\Gamma_{\text {ind }}[g, \hat{g}] \equiv & \frac{1}{96 \pi} \int(\sqrt{g} R+\sqrt{\hat{g}} \hat{R}) \square^{-1} \frac{1}{\sqrt{g}}(\sqrt{g} R-\sqrt{\hat{g}} \hat{R}) \\
& -\frac{\chi}{12 V} \int \sqrt{g} \ln \left(\frac{\sqrt{g}}{\sqrt{\hat{g}}}\right)+\frac{1}{2} \ln \left(\frac{V}{\hat{V}}\right) .
\end{aligned}
$$


In this expression it does not seem possible to disentangle $g$ from $\hat{g}$.

Nevertheless, by introducing a fiducial metric $g_{0}$ in (A.33) we could define $\Gamma_{\text {ind }}[g]$ formally up to an additive constant by

$$
\Gamma_{\text {ind }}[g] \equiv \Gamma_{\text {ind }}\left[g, g_{0}\right]
$$

Using this definition, $\Gamma_{\text {ind }}$ satisfies indeed eq. (A.29). The corresponding functional $I_{\text {full }}[g]$ (where $I_{\text {full }}$ refers to the general case, with zero mode and arbitrary rescalings) can be obtained by applying rule (A.19), $\left.I_{\text {full }}[g] \equiv 96 \pi \Gamma_{\text {ind }}[g]\right|_{\text {finite }}$, resulting in

$$
I_{\text {full }}[g] \equiv I[g]+R\left[g, g_{0}\right],
$$

with $I[g]=\int \sqrt{g} R \square^{-1} R$ as above, and with the residue

$$
R\left[g, g_{0}\right] \equiv-\int \sqrt{g_{0}} R\left(g_{0}\right) \square^{-1} \frac{\sqrt{g_{0}}}{\sqrt{g}} R\left(g_{0}\right)-\frac{8 \pi \chi}{V} \int \sqrt{g} \ln \left(\frac{\sqrt{g}}{\sqrt{g_{0}}}\right)+48 \pi \ln \left(\frac{V}{V_{0}}\right) .
$$

This residue is due to the zero mode contribution to the conformal factor relating $g$ with $g_{0}$. Using eq. (A.29) leads to a transformation behavior of $I_{\text {full }}[g]$ similar to the one found in section A.1. We obtain

$$
I_{\text {full }}[g]-I_{\text {full }}[\hat{g}]=-8 \Delta I[\sigma ; \hat{g}]+48 \pi \ln (V / \hat{V}) .
$$

Thus, apart from the pure volume terms we recover the same result as in eq. (A.21), the modification being due to the zero modes of $\square$ and $\hat{\square}, \varphi^{(0)}=1 / \sqrt{V}$ and $\hat{\varphi}^{(0)}=1 / \sqrt{\hat{V}}$, respectively.

Concerning our results of section 3 we observe that $I[g]$ is to be replaced according to

$$
I[g] \rightarrow I_{\text {full }}[g]-48 \pi \ln \left(V / V_{0}\right)
$$

where the corresponding behavior under Weyl transformations is given by eq. (A.37). Thus, in the general case there are additional correction terms in consequence of the zero modes. In particular, eq. (3.29) generalizes to

$$
\frac{1}{\varepsilon} \int \mathrm{d}^{2+\varepsilon} x \sqrt{g} R=-\frac{1}{4} I[g]+Q\left[g, g_{0}\right]+\frac{4 \pi \chi}{\varepsilon}+C(\{\tau\})+\mathcal{O}(\varepsilon)
$$

with the correction terms $Q\left[g, g_{0}\right] \equiv \frac{1}{4} \int \sqrt{g_{0}} R\left(g_{0}\right) \square^{-1} \frac{\sqrt{g_{0}}}{\sqrt{g}} R\left(g_{0}\right)+\frac{2 \pi \chi}{V} \int \sqrt{g} \ln \left(\frac{\sqrt{g}}{\sqrt{g_{0}}}\right)$. We would like to point out that the crucial result in eq. (3.29), the appearance of the non-local action $I[g]$, is contained in its extension (A.39), too. All conclusions in the main part of this paper that relied on the emergence of $I[g]$ in the 2D limit of the Einstein-Hilbert action remain valid in the presence of zero modes. The correction terms in (A.39) do not change our main results, in particular the central charge, which is read off from the prefactor of $I[g]$, remains unaltered.

Finally, two comments are in order. 
(1) Nonvanishing Euler characteristics. We would like to point out the following subtlety concerning the induced gravity functional $I$. As argued above, $\square^{-1}$ is defined such that it affects only nonzero modes while it "projects away" the zero modes of the objects it acts on. In particular, the function $\left(\square^{-1} R\right)(x)$ satisfies $\square \square^{-1} R=R-\frac{1}{V} 4 \pi \chi$. Hence, for manifolds with vanishing Euler characteristic, $\chi=0$, we recover the usual feature of an inverse operator, $\square \square^{-1} R=R$, as long as $\square^{-1}$ acts on $R$. The reason behind this property is that the Fourier expansion of $R$ cannot contain any contribution $\propto c_{0} \varphi^{(0)}$ from the zero mode if $\chi=0$. As a consequence $\square^{-1} R$ is non-zero provided that $R$ does not vanish, and, in turn, $I[g]$ is a non-zero functional.

On the other hand, if $\chi \neq 0$, then it might happen that $I[g]$ vanishes. As an example, let us consider a sphere with constant curvature $R>0$. Since $R$ is proportional to the constant zero mode in this case, we have $\square^{-1} R=0$, and thus $I[g]=0$. With regard to eq. (A.37) this means that all nontrivial contributions to the l.h.s. must come from $I_{\text {full }}[\hat{g}]$ and from the residue contained in $I_{\text {full }}[g]$.

(2) A modified induced gravity functional. The occurrence of the volume term in eq. (A.37) can be understood as follows. We removed the zero modes from the path integral (A.25), and this exclusion affects the transformation behavior, replacing (A.21) with (A.37). However, there is the possibility to redefine the partition function in order to absorb the volume terms. Let us briefly sketch the idea.

As above we expand the scalar field $X$ in the partition function in terms of normalized eigenmodes $\varphi^{(n)}$ of the Laplacian, $X=\sum_{n} c_{n} \varphi^{(n)}$, and insert this into eq. (A.25). Then it is easy to show (see e.g. [122]) that the transformation behavior of $\ln Z$ under a Weyl variation according to eq. (A.1), $\delta g_{\mu \nu}=2 \sigma g_{\mu \nu}$, is given by

$$
\delta \ln Z=\int \mathrm{d}^{2} x \sqrt{g}\left(\frac{1}{4} \frac{\delta g}{g}\right) \sum_{n=0}^{\infty}\left[\varphi^{(n)}\right]^{2}-\frac{1}{2} \frac{\delta V}{V} .
$$

Rearranging terms yields

$$
\delta \ln \left(\sqrt{V / V_{0}} Z\right)=\int \mathrm{d}^{2} x \sqrt{g}\left(\frac{1}{4} \frac{\delta g}{g}\right) \sum_{n=0}^{\infty}\left[\varphi^{(n)}\right]^{2},
$$

where $V_{0}$ is an arbitrary reference volume introduced merely to render the argument of the logarithm dimensionless. The advantage of eq. (A.41) is that its r.h.s. does no longer contain any distinction between zero and nonzero modes, so the combination $\sqrt{V / V_{0}} Z$ is more appropriate for a treatment of all modes on an equal footing.

These observations suggest introducing the modified definition

$$
Z_{\text {mod }}[g] \equiv \sqrt{V / V_{0}} \int \mathcal{D}^{\prime} X \mathrm{e}^{-\frac{1}{2} \int \mathrm{d}^{2} x \sqrt{g} g^{\mu \nu} \partial_{\mu} X \partial_{\nu} X}
$$

The corresponding effective action reads

$$
\Gamma_{\text {ind,mod }}[g]=\frac{1}{2} \ln \operatorname{det}^{\prime}(-\square)-\frac{1}{2} \ln \frac{V}{V_{0}} .
$$


This modified effective action is often used in the literature [123]. Applying the rule (A.19) to (A.43) and using (A.35) yields the modified induced gravity functional

$$
I_{\text {mod }}[g] \equiv I_{\text {full }}[g]-48 \pi \ln \frac{V}{V_{0}},
$$

consistent with (A.38). Employing eq. (A.37) we find that it transforms according to

$$
I_{\text {mod }}[g]-I_{\bmod }[\hat{g}]=-8 \Delta I[\sigma ; \hat{g}],
$$

with $\Delta I$ as defined in eq. (A.22). Thus, for $I_{\bmod }[g]$ we recover the same behavior under Weyl transformations as for $I[g]$ in eq. (A.21), which was the transformation law for the case without zero modes.

In conclusion, zero modes can be taken into account by employing a modified definition of the path integral, where the behavior of the (generalized) induced gravity functional under Weyl rescalings remains essentially the same.

Open Access. This article is distributed under the terms of the Creative Commons Attribution License (CC-BY 4.0), which permits any use, distribution and reproduction in any medium, provided the original author(s) and source are credited.

\section{References}

[1] S. Weinberg, Ultraviolet divergences in quantum theories of gravitation, in General relativity, an Einstein centenary survey, S.W. Hawking and W. Israel eds., Cambridge University Press, Cambridge U.K. (1980), pg. 790.

[2] Y. Nakayama, Scale invariance vs conformal invariance, Phys. Rept. 569 (2015) 1 [arXiv: 1302.0884] [INSPIRE].

[3] D. Becker and M. Reuter, En route to background independence: broken split-symmetry and how to restore it with bi-metric average actions, Annals Phys. 350 (2014) 225 [arXiv:1404.4537] [INSPIRE].

[4] K. Osterwalder and R. Schrader, Axioms for Euclidean Green's functions, Commun. Math. Phys. 31 (1973) 83 [INSPIRE].

[5] K. Osterwalder and R. Schrader, Axioms for Euclidean Green's functions. 2, Commun. Math. Phys. 42 (1975) 281 [INSPIRE].

[6] F. Strocchi, Selected topics on the general properties of quantum field theory, World Scientific, Singapore (1993).

[7] J. Glimm and A. Jaffe, Quantum physics, Springer, New York U.S.A. (1987).

[8] M. Reuter, Nonperturbative evolution equation for quantum gravity, Phys. Rev. D 57 (1998) 971 [hep-th/9605030] [INSPIRE].

[9] E. Manrique and M. Reuter, Bare action and regularized functional integral of asymptotically safe quantum gravity, Phys. Rev. D 79 (2009) 025008 [arXiv:0811.3888] [INSPIRE].

[10] G.P. Vacca and L. Zambelli, Functional $R G$ flow equation: regularization and coarse-graining in phase space, Phys. Rev. D 83 (2011) 125024 [arXiv:1103.2219] [INSPIRE]. 
[11] T.R. Morris, The exact renormalization group and approximate solutions, Int. J. Mod. Phys. A 9 (1994) 2411 [hep-ph/9308265] [INSPIRE].

[12] T.R. Morris and Z.H. Slade, Solutions to the reconstruction problem in asymptotic safety, JHEP 11 (2015) 094 [arXiv: 1507.08657] [INSPIRE].

[13] M. Reuter and C. Wetterich, Average action for the Higgs model with Abelian gauge symmetry, Nucl. Phys. B 391 (1993) 147 [inSPIRE].

[14] M. Reuter and C. Wetterich, Running gauge coupling in three-dimensions and the electroweak phase transition, Nucl. Phys. B 408 (1993) 91 [INSPIRE].

[15] C. Wetterich, Exact evolution equation for the effective potential, Phys. Lett. B 301 (1993) 90 [INSPIRE].

[16] M. Reuter and C. Wetterich, Effective average action for gauge theories and exact evolution equations, Nucl. Phys. B 417 (1994) 181 [inSPIRE].

[17] M. Reuter and C. Wetterich, Exact evolution equation for scalar electrodynamics, Nucl. Phys. B 427 (1994) 291 [inSPIRE].

[18] M. Niedermaier and M. Reuter, The asymptotic safety scenario in quantum gravity, Living Rev. Rel. 9 (2006) 5.

[19] R. Percacci, Asymptotic safety, in Approaches to quantum gravity: towards a new understanding of space, time and matter, D. Oriti ed., Cambridge University Press, Cambridge U.K. (2009) [arXiv: 0709.3851] [INSPIRE].

[20] A. Codello, R. Percacci and C. Rahmede, Investigating the ultraviolet properties of gravity with a Wilsonian renormalization group equation, Annals Phys. 324 (2009) 414 [arXiv:0805.2909] [INSPIRE].

[21] M. Reuter and F. Saueressig, Quantum Einstein gravity, New J. Phys. 14 (2012) 055022 [arXiv:1202.2274] [INSPIRE].

[22] A. Codello and G. D'Odorico, Scaling and renormalization in two dimensional quantum gravity, Phys. Rev. D 92 (2015) 024026 [arXiv:1412.6837] [INSPIRE].

[23] E. Manrique and M. Reuter, Bimetric truncations for quantum Einstein gravity and asymptotic safety, Annals Phys. 325 (2010) 785 [arXiv:0907.2617] [INSPIRE].

[24] A. Nink and M. Reuter, On the physical mechanism underlying asymptotic safety, JHEP 01 (2013) 062 [arXiv:1208.0031] [INSPIRE].

[25] A. Nink and M. Reuter, On quantum gravity, asymptotic safety and paramagnetic dominance, arXiv: 1212.4325 [INSPIRE].

[26] A. Nink, Field parametrization dependence in asymptotically safe quantum gravity, Phys. Rev. D 91 (2015) 044030 [arXiv:1410.7816] [INSPIRE].

[27] A.M. Polyakov, Quantum geometry of bosonic strings, Phys. Lett. B 103 (1981) 207 [INSPIRE].

[28] M. Reuter and C. Wetterich, Quantum Liouville field theory as solution of a flow equation, Nucl. Phys. B 506 (1997) 483 [hep-th/9605039] [INSPIRE].

[29] A. Nink and M. Reuter, The reconstruction problem in 2D Asymptotic Safety, in preparation. 
[30] Y. Imayoshi and M. Taniguchi, An introduction to Teichmüller spaces, Springer, Tokyo Japan (1992).

[31] B.A. Dubrovin, A.T. Fomenko and S.P. Novikov, Modern geometry - methods and applications, $2^{\text {nd }}$ edition, Springer, New York U.S.A. (1992).

[32] J. Polchinski, String theory - volume 1, Cambridge University Press, Cambridge U.K. (1998).

[33] H. Yamabe, On a deformation of Riemannian structures on compact manifolds, Osaka J. Math. 12 (1960) 21.

[34] N.S. Trudinger, Remarks concerning the conformal deformation of Riemannian structures on compact manifolds, Ann. Scuola Norm. Sup. Pisa 22 (1968) 265.

[35] T. Aubin, Métriques riemanniennes et courbure (in French), J. Diff. Geom. 4 (1970) 383.

[36] T. Aubin, Equations différentielles nonlinéaires et problème de Yamabe concernant la courbure scalaire (in French), J. Math. Pures Appl. 55 (1976) 269.

[37] R. Schoen, Conformal deformation of a Riemannian metric to constant scalar curvature, J. Diff. Geom. 20 (1984) 479.

[38] P. Nurowski and J.F. Plebanski, Nonvacuum twisting type $N$ metrics, Class. Quant. Grav. 18 (2001) 341 [gr-qc/0007017] [INSPIRE].

[39] D.M. Capper and D. Kimber, An ambiguity in one loop quantum gravity, J. Phys. A 13 (1980) 3671 [inSPIRE].

[40] R.B. Mann and S.F. Ross, The $D \rightarrow 2$ limit of general relativity, Class. Quant. Grav. 10 (1993) 1405 [gr-qc/9208004] [INSPIRE].

[41] R. Jackiw, Weyl symmetry and the Liouville theory, Theor. Math. Phys. 148 (2006) 941 [Teor. Mat. Fiz. 148 (2006) 80] [hep-th/0511065] [INSPIRE].

[42] D. Grumiller and R. Jackiw, Liouville gravity from Einstein gravity, in Recent developments in theoretical physics, S. Gosh and G. Kar eds., World Scientific, Singapore (2010), pg. 331 [arXiv:0712.3775] [INSPIRE].

[43] P.O. Mazur and E. Mottola, Weyl cohomology and the effective action for conformal anomalies, Phys. Rev. D 64 (2001) 104022 [hep-th/0106151] [INSPIRE].

[44] H.-S. Tsao, Conformal anomalies in a general background metric, Phys. Lett. B 68 (1977) 79 [inSPIRE].

[45] L.S. Brown, Stress tensor trace anomaly in a gravitational metric: scalar fields, Phys. Rev. D 15 (1977) 1469 [InSPIRE].

[46] R. Gastmans, R. Kallosh and C. Truffin, Quantum gravity near two-dimensions, Nucl. Phys. B 133 (1978) 417 [inSPIRE].

[47] S.M. Christensen and M.J. Duff, Quantum gravity in two $+\epsilon$ dimensions, Phys. Lett. B 79 (1978) 213 [INSPIRE].

[48] H. Kawai and M. Ninomiya, Renormalization group and quantum gravity, Nucl. Phys. B 336 (1990) 115 [inSPIRE].

[49] I. Jack and D.R.T. Jones, The $\epsilon$-expansion of two-dimensional quantum gravity, Nucl. Phys. B 358 (1991) 695 [InSPIRE]. 
[50] H. Kawai, Y. Kitazawa and M. Ninomiya, Quantum gravity in 2+ dimensions, Prog. Theor. Phys. Supp. 114 (1993) 149.

[51] H. Kawai, Y. Kitazawa and M. Ninomiya, Scaling exponents in quantum gravity near two-dimensions, Nucl. Phys. B 393 (1993) 280 [hep-th/9206081] [INSPIRE].

[52] H. Kawai, Y. Kitazawa and M. Ninomiya, Ultraviolet stable fixed point and scaling relations in $(2+\epsilon)$-dimensional quantum gravity, Nucl. Phys. B 404 (1993) 684 [hep-th/9303123] [INSPIRE].

[53] H. Kawai, Y. Kitazawa and M. Ninomiya, Renormalizability of quantum gravity near two-dimensions, Nucl. Phys. B 467 (1996) 313 [hep-th/9511217] [INSPIRE].

[54] T. Aida, Y. Kitazawa, H. Kawai and M. Ninomiya, Conformal invariance and renormalization group in quantum gravity near two-dimensions,

Nucl. Phys. B 427 (1994) 158 [hep-th/9404171] [INSPIRE].

[55] J. Nishimura, S. Tamura and A. Tsuchiya, $R^{2}$ gravity in $(2+\epsilon)$-dimensional quantum gravity, Mod. Phys. Lett. A 9 (1994) 3565 [hep-th/9405059] [INSPIRE].

[56] T. Aida and Y. Kitazawa, Two loop prediction for scaling exponents in $(2+\epsilon)$-dimensional quantum gravity, Nucl. Phys. B 491 (1997) 427 [hep-th/9609077] [INSPIRE].

[57] K. Falls, Renormalization of Newton's constant, Phys. Rev. D 92 (2015) 124057 [arXiv: 1501.05331] [INSPIRE].

[58] K. Falls, Critical scaling in quantum gravity from the renormalisation group, arXiv: 1503.06233 [INSPIRE].

[59] M. Demmel and A. Nink, Connections and geodesics in the space of metrics, Phys. Rev. D 92 (2015) 104013 [arXiv:1506.03809] [InSPIRE].

[60] J.P.S. Lemos and P.M. Sa, The two-dimensional analog of general relativity, Class. Quant. Grav. 11 (1994) L11 [gr-qc/9310041] [INSPIRE].

[61] N. Ohta and R. Percacci, Higher derivative gravity and asymptotic safety in diverse dimensions, Class. Quant. Grav. 31 (2014) 015024 [arXiv:1308.3398] [INSPIRE].

[62] A.H. Chamseddine and M. Reuter, Induced two-dimensional quantum gravity and $\operatorname{SL}(2, R)$ Kac-Moody current algebra, Nucl. Phys. B 317 (1989) 757 [INSPIRE].

[63] E. D'Hoker, Lecture notes on 2D quantum gravity and Liouville theory, in Proceedings, Particle physics, Campos do Jordao Brazil (1991), pg. 282 [UCLA-91-TEP-35] [INSPIRE].

[64] P.H. Ginsparg and G.W. Moore, Lectures on $2 D$ gravity and $2 D$ string theory, in Proceedings, Recent directions in particle theory, Boulder U.S.A. (1992), pg. 277 [hep-th/9304011] [INSPIRE].

[65] Y. Nakayama, Liouville field theory: a decade after the revolution, Int. J. Mod. Phys. A 19 (2004) 2771 [hep-th/0402009] [INSPIRE].

[66] E. Abdalla, M.C.B. Abdalla, D. Dalmazi and A. Zadra, 2D-gravity in non-critical strings: discrete and continuum approaches, Springer, New York U.S.A. (1994).

[67] A. Codello, G. D'Odorico and C. Pagani, A functional RG equation for the c-function, JHEP 07 (2014) 040 [arXiv: 1312.7097] [INSPIRE].

[68] A. Chodos and C.B. Thorn, Making the massless string massive, Nucl. Phys. B 72 (1974) 509 [inSPIRE]. 
[69] V.S. Dotsenko and V.A. Fateev, Conformal algebra and multipoint correlation functions in two-dimensional statistical models, Nucl. Phys. B 240 (1984) 312 [INSPIRE].

[70] V.S. Dotsenko and V.A. Fateev, Four point correlation functions and the operator algebra in the two-dimensional conformal invariant theories with the central charge $c<1$,

Nucl. Phys. B 251 (1985) 691 [inSPIRE].

[71] P. Di Francesco, P. Mathieu and D. Sénéchal, Conformal field theory, Springer, New York U.S.A. (1997).

[72] G. Mussardo, Statistical field theory: an introduction to exactly solved models in statistical physics, Oxford University Press, Oxford U.K. (2010).

[73] M. Schottenloher, A mathematical introduction to conformal field theory, Springer, Berlin Germany (2008).

[74] S.L. Adler, Einstein gravity as a symmetry breaking effect in quantum field theory, Rev. Mod. Phys. 54 (1982) 729 [Erratum ibid. 55 (1983) 837] [InSPIRE].

[75] J. Polchinski, A two-dimensional model for quantum gravity, Nucl. Phys. B 324 (1989) 123 [INSPIRE].

[76] J.D. Bjorken and S.D. Drell, Relativistic quantum fields, McGraw-Hill, New York U.S.A. (1965).

[77] E. Gozzi, E. Cattaruzza and C. Pagani, Path integrals for pedestrians, World Scientific, Singapore (2015).

[78] E. Witten, $(2+1)$-dimensional gravity as an exactly soluble system, Nucl. Phys. B 311 (1988) 46 [INSPIRE].

[79] J. Ambjørn and R. Loll, Nonperturbative Lorentzian quantum gravity, causality and topology change, Nucl. Phys. B 536 (1998) 407 [hep-th/9805108] [INSPIRE].

[80] H. Gies, B. Knorr and S. Lippoldt, Generalized parametrization dependence in quantum gravity, Phys. Rev. D 92 (2015) 084020 [arXiv: 1507.08859] [INSPIRE].

[81] N. Ohta, R. Percacci and G.P. Vacca, Flow equation for $f(R)$ gravity and some of its exact solutions, Phys. Rev. D 92 (2015) 061501 [arXiv: 1507.00968] [INSPIRE].

[82] N. Ohta, R. Percacci and G.P. Vacca, Renormalization group equation and scaling solutions for $f(R)$ gravity in exponential parametrization, arXiv:1511.09393 [INSPIRE].

[83] M. Reuter and H. Weyer, Background independence and asymptotic safety in conformally reduced gravity, Phys. Rev. D 79 (2009) 105005 [arXiv:0801.3287] [INSPIRE].

[84] M. Reuter and H. Weyer, Conformal sector of quantum Einstein gravity in the local potential approximation: non-Gaussian fixed point and a phase of unbroken diffeomorphism invariance, Phys. Rev. D 80 (2009) 025001 [arXiv:0804.1475] [InSPIRE].

[85] R. Jackiw, C. Núñez and S.-Y. Pi, Quantum relaxation of the cosmological constant, Phys. Lett. A 347 (2005) 47 [hep-th/0502215] [INSPIRE].

[86] P.F. Machado and R. Percacci, Conformally reduced quantum gravity revisited, Phys. Rev. D 80 (2009) 024020 [arXiv:0904.2510] [INSPIRE].

[87] H.-J. Otto and G. Weigt, Construction of exponential Liouville field operators for closed string models, Z. Phys. C 31 (1986) 219 [INSPIRE]. 
[88] H. Dorn and H.-J. Otto, Two and three point functions in Liouville theory, Nucl. Phys. B 429 (1994) 375 [hep-th/9403141] [INSPIRE].

[89] Y. Kazama and H. Nicolai, On the exact operator formalism of two-dimensional Liouville quantum gravity in Minkowski space-time, Int. J. Mod. Phys. A 9 (1994) 667 [hep-th/9305023] [INSPIRE].

[90] P. Dona and R. Percacci, Functional renormalization with fermions and tetrads, Phys. Rev. D 87 (2013) 045002 [arXiv:1209.3649] [InSPIRE].

[91] P. Donà, A. Eichhorn and R. Percacci, Matter matters in asymptotically safe quantum gravity, Phys. Rev. D 89 (2014) 084035 [arXiv:1311.2898] [INSPIRE].

[92] F. David, Conformal field theories coupled to $2 D$ gravity in the conformal gauge, Mod. Phys. Lett. A 3 (1988) 1651 [INSPIRE].

[93] J. Distler and H. Kawai, Conformal field theory and 2D quantum gravity or who's afraid of Joseph Liouville?, Nucl. Phys. B 321 (1989) 509 [INSPIRE].

[94] Y. Watabiki, Analytic study of fractal structure of quantized surface in two-dimensional quantum gravity, Prog. Theor. Phys. Suppl. 114 (1993) 1 [INSPIRE].

[95] A.M. Polyakov, Quantum gravity in two-dimensions, Mod. Phys. Lett. A 2 (1987) 893 [INSPIRE].

[96] V.G. Knizhnik, A.M. Polyakov and A.B. Zamolodchikov, Fractal structure of $2 D$ quantum gravity, Mod. Phys. Lett. A 3 (1988) 819 [inSPIRE].

[97] F. David and E. Guitter, Instabilities in membrane models, Europhys. Lett. 3 (1987) 1169 [INSPIRE].

[98] F. David and E. Guitter, Rigid random surfaces at large d, Nucl. Phys. B 295 (1988) 332 [INSPIRE].

[99] S.R. Das, S. Naik and S.R. Wadia, Quantization of the Liouville mode and string theory, Mod. Phys. Lett. A 4 (1989) 1033 [inSPIRE].

[100] J.-E. Daum and M. Reuter, Effective potential of the conformal factor: gravitational average action and dynamical triangulations, Adv. Sci. Lett. 2 (2009) 255 [arXiv:0806.3907] [INSPIRE].

[101] O. Lauscher and M. Reuter, Fractal spacetime structure in asymptotically safe gravity, JHEP 10 (2005) 050 [hep-th/0508202] [INSPIRE].

[102] M. Reuter and F. Saueressig, Fractal space-times under the microscope: a renormalization group view on Monte Carlo data, JHEP 12 (2011) 012 [arXiv:1110.5224] [INSPIRE].

[103] J. Ambjørn, A. Görlich, J. Jurkiewicz and R. Loll, Nonperturbative quantum gravity, Phys. Rept. 519 (2012) 127 [arXiv:1203.3591] [INSPIRE].

[104] J. Ambjørn, K.N. Anagnostopoulos and R. Loll, Crossing the $c=1$ barrier in $2 D$ Lorentzian quantum gravity, Phys. Rev. D 61 (2000) 044010 [hep-lat/9909129] [InSPIRE].

[105] J. Ambjørn, K.N. Anagnostopoulos, R. Loll and I. Pushkina, Shaken, but not stirred: Potts model coupled to quantum gravity, Nucl. Phys. B 807 (2009) 251 [arXiv:0806.3506] [INSPIRE].

[106] J. Ambjørn, A. Görlich, J. Jurkiewicz and H. Zhang, The spectral dimension in $2 D C D T$ gravity coupled to scalar fields, Mod. Phys. Lett. A 30 (2015) 1550077 [arXiv:1412.3434] [INSPIRE]. 
[107] J. Ambjørn, A. Görlich, J. Jurkiewicz and H. Zhang, A $c=1$ phase transition in two-dimensional CDT/Hořava-Lifshitz gravity?, Phys. Lett. B 743 (2015) 435 [arXiv: 1412.3873] [INSPIRE].

[108] J. Ambjørn, A. Görlich, J. Jurkiewicz and H. Zhang, The microscopic structure of $2 D$ CDT coupled to matter, Phys. Lett. B 746 (2015) 359 [arXiv:1503.01636] [inSPIRE].

[109] J.-L. Gervais, Critical dimensions for noncritical strings, Phys. Lett. B 243 (1990) 85 [INSPIRE].

[110] J.L. Gervais, Solving the strongly coupled 2D gravity: 1. Unitary truncation and quantum group structure, Commun. Math. Phys. 138 (1991) 301 [INSPIRE].

[111] J.L. Gervais, On the algebraic structure of quantum gravity in two-dimensions, Int. J. Mod. Phys. A 6 (1991) 2805 [inSPIRE].

[112] J.L. Gervais, Physical features of strongly coupled 2D gravity, Phys. Lett. B 255 (1991) 22 [INSPIRE].

[113] J.-L. Gervais, The new physics of strongly coupled $2 D$ gravity, in Future perspectives in string theory: strings 95, USC, Los Angeles U.S.A. (1995), pg. 200 [hep-th/9506040] [INSPIRE].

[114] J.-L. Gervais, Chirality deconfinement beyond the $c=1$ barrier of two-dimensional gravity, in Low-dimensional applications of quantum field theory, NATO ASI series, Cargese France, Springer Germany (1997), pg. 145 [hep-th/9606151] [INSPIRE].

[115] A.O. Barvinsky and G.A. Vilkovisky, Beyond the Schwinger-Dewitt technique: converting loops into trees and in-in currents, Nucl. Phys. B 282 (1987) 163 [INSPIRE].

[116] A.O. Barvinsky and G.A. Vilkovisky, Covariant perturbation theory. 2: second order in the curvature. General algorithms, Nucl. Phys. B 333 (1990) 471 [INSPIRE].

[117] A.O. Barvinsky and G.A. Vilkovisky, Covariant perturbation theory. 3: spectral representations of the third order form-factors, Nucl. Phys. B 333 (1990) 512 [INSPIRE].

[118] G.A. Vilkovisky, Heat kernel: rencontre entre physiciens et mathématiciens (in French), in Meeting between Physicists and Mathematicians, Strasbourg France December 1991, CERN-TH-6392-92, (1992).

[119] D. Fursaev and D. Vassilevich, Operators, geometry and quanta, Springer, Germany (2011).

[120] J.S. Dowker, A note on Polyakov's nonlocal form of the effective action, Class. Quant. Grav. 11 (1994) L7 [hep-th/9309127] [INSPIRE].

[121] M.J. Duff, Twenty years of the Weyl anomaly, Class. Quant. Grav. 11 (1994) 1387 [hep-th/9308075] [INSPIRE].

[122] C. Itzykson and J.-M. Drouffe, Statistical field theory, volume 2, Cambridge University Press, Cambridge U.K. (1989).

[123] S. Chaudhuri, H. Kawai and S.-H. Henry Tye, Path integral formulation of closed strings, Phys. Rev. D 36 (1987) 1148 [INSPIRE]. 